\title{
A closer look at fracture healing
}

Citation for published version (APA):

de Jong, J. J. A. (2017). A closer look at fracture healing: fracture healing at the distal assessed using high-resolution peripheral quantitative computed tomography. [Doctoral Thesis, Maastricht University]. Maastricht University. https://doi.org/10.26481/dis.20170113jdj

Document status and date:

Published: 01/01/2017

DOI:

10.26481/dis.20170113jdj

Document Version:

Publisher's PDF, also known as Version of record

\section{Please check the document version of this publication:}

- A submitted manuscript is the version of the article upon submission and before peer-review. There can be important differences between the submitted version and the official published version of record.

People interested in the research are advised to contact the author for the final version of the publication, or visit the DOI to the publisher's website.

- The final author version and the galley proof are versions of the publication after peer review.

- The final published version features the final layout of the paper including the volume, issue and page numbers.

Link to publication

\footnotetext{
General rights rights.

- You may freely distribute the URL identifying the publication in the public portal. please follow below link for the End User Agreement:

www.umlib.nl/taverne-license

Take down policy

If you believe that this document breaches copyright please contact us at:

repository@maastrichtuniversity.nl

providing details and we will investigate your claim.
}

Copyright and moral rights for the publications made accessible in the public portal are retained by the authors and/or other copyright owners and it is a condition of accessing publications that users recognise and abide by the legal requirements associated with these

- Users may download and print one copy of any publication from the public portal for the purpose of private study or research.

- You may not further distribute the material or use it for any profit-making activity or commercial gain

If the publication is distributed under the terms of Article $25 \mathrm{fa}$ of the Dutch Copyright Act, indicated by the "Taverne" license above, 


\section{A Closer Look at Fracture Healing}

Fracture healing at the distal radius assessed using high-resolution peripheral quantitative computed tomography 
Copyright $\odot$ J.J.A. de Jong, Maastricht 2016

ISBN: 978-94-6332-116-7

Layout and cover design: Citroenvlinder DTP \& Vormgeving, my-thesis.nl Printed by: GVO drukkers en vormgevers B.V.

For all articles published, the copyright has been transferred to the respective publisher. No part of this thesis may be reproduced in any form or by any means without written permission from the author or, when appropriate, from the publisher.

Printing of this thesis was financially supported by Maastricht University, Scanco Medical AG, Amgen B.V., Will-Pharma B.V., Pearltec AG and Icotec AG. 


\section{A Closer Look at Fracture Healing}

Fracture healing at the distal radius assessed using

high-resolution peripheral quantitative computed tomography

\section{PROEFSCHRIFT}

ter verkrijging van de graad van doctor aan de Universiteit Maastricht, op gezag van de Rector Magnificus, Prof. dr. Rianne M. Letschert volgens het besluit van het College van Decanen,

in het openbaar te verdedigen

op vrijdag 13 januari 2017 om 12.00 uur

door

Joost Johannes Antoin de Jong

Geboren op 21 december 1984 te Hulst 


\section{Promotoren}

Prof. dr. P.P.M.M. Geusens

Prof. dr. J.P.W. van den Bergh

\section{Copromotoren}

Dr. P.C. Willems

Dr. ir. B. van Rietbergen (Technische Universiteit Eindhoven)

\section{Beoordelingscommissie}

Prof. dr. L. van Rhijn (voorzitter)

Prof. dr. K. Ito (Technische Universiteit Eindhoven)

Prof. dr. H. van Lenthe (Katholieke Universiteit Leuven)

Dr. R.E. Weijers 


\section{Contents}

List of abbreviations $\quad 6$

1 General introduction 9

Part 1 Assessment of stable distal radius fracture healing using HRpQCT 19

2 Effect of casting on short-term reproducibility and bone parameters 21 obtained from HRpQCT measurements at the distal radius.J Bone Joint Surg Am 98, 356-62 (2016)

3 Assessment of the healing process in distal radius fractures by high-resolution peripheral quantitative computed tomography. Bone 64, 65-74 (2014)

4 Early changes in bone density, micro-architecture, bone resorption and inflammation predict the clinical outcome twelve weeks after conservatively treated distal radius fractures. J Bone Min Res 29, 2065-73 (2014)

Part 2 Follow-up two years after a stable distal radius fracture using HRpQCT

5 Fracture repair in the distal radius in post-menopausal women: a follow-up two years post-fracture using HRpQCT. J Bone Min Res 31, 1114$22(2016)$

6 Contra-Lateral bone loss at the distal radius in postmenopausal women after a distal radius fracture: a two-year follow-up HRpQCT Study. Submitted

Part 3 Applicability of HRpQCT in unstable distal radius fractures

7 Feasibility of rigid 3D image registration of high-resolution peripheral quantitative computed tomography images of healing distal radius fractures. Submitted

8 Distal radius plate of CFR-PEEK has minimal effect on bone parameters in high-resolution peripheral quantitative computed tomography: a pilot study. Submitted

9 General discussion $\quad 151$

Valorization 164

Summary 166

Samenvatting 170

$\begin{array}{ll}\text { Dankwoord } & 176\end{array}$

Curriculum vitae $\quad 181$

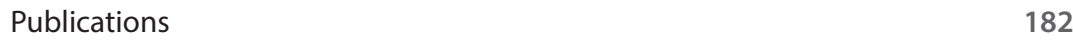




\section{List of abbreviations}

\begin{tabular}{|c|c|}
\hline$\mu \mathrm{FEA}$ & micro finite element analysis \\
\hline $25(\mathrm{OH}) \mathrm{D}$ & 25-hydroxyvitamin D \\
\hline $2 \mathrm{D} / 3 \mathrm{D}$ & two/three-dimensional \\
\hline aBMD & areal bone mineral density \\
\hline AP & anteroposterior \\
\hline $\mathrm{BMI}$ & body mass index \\
\hline CFR-PEEK & carbon-fiber-reinforced polyetheretherketone \\
\hline $\mathrm{Cl}$ & confidence interval \\
\hline Ct.Pm & cortical perimeter \\
\hline Ct.Po & cortical porosity \\
\hline Ct.Po.D & cortical pore diameter \\
\hline Ct.Po.V & cortical pore volume \\
\hline Ct.Th & cortical thickness \\
\hline Dcort & cortical density \\
\hline Dtot & total density \\
\hline Dtrab & trabecular density \\
\hline DXA & dual-energy X-ray absorptiometry \\
\hline F.Ult & estimated ultimate failure load \\
\hline FLS & fracture liaison service \\
\hline $\mathrm{HA}$ & hydroxyapatite \\
\hline HRpQCT & high-resolution peripheral quantitative computed tomography \\
\hline hsCRP & high-sensitivity C-reactive protein \\
\hline ICTP & carboxy-terminal telopeptide of type I collagen \\
\hline LSC & least significant change \\
\hline PINP & procollagen type-I N-terminal propeptide \\
\hline PRWE & patient-rated wrist evaluation \\
\hline PTH & parathyroid hormone \\
\hline PVE & partial-volume effect \\
\hline QCT & quantitative computed tomography \\
\hline QuickDASH & quick disabilities of the arm, shoulder and hand \\
\hline RMS-CV & root-mean-square coefficient of variation \\
\hline ROI & region of interest \\
\hline RSA & radiostereometric analysis \\
\hline Sbend & bending stiffness \\
\hline Scomp & compression stiffness \\
\hline SD & standard deviation \\
\hline Stors & torsional stiffness \\
\hline
\end{tabular}


Tb.N

Tb.Sp

Tb.Th

TSH

VAS

VBMD

VDRP

VF trabecular number

trabecular separation

trabecular thickness

thyroid stimulating hormone

visual analogue scale

volumetric bone mineral density

volar distal radius plate

vertebral fracture 


$$
\frac{b}{x}
$$


General introduction 


\section{Scope}

One of the most common type of fractures is a fracture of the distal radius. Especially post-menopausal women, in whom the incidence of distal radius fractures is four times higher than in men, are vulnerable: from 1997 to 2009, the annual incidence in The Netherlands of wrist fractures in women aged 50 years or older was 458 per 100.000 persons. ${ }^{1}$

Depending on the degree of instability and comminution, distal radius fractures are treated conservatively by cast immobilization, or surgically by internal or external fixation. ${ }^{2}$ When treated by a cast, there is usually sufficient stability of the fracture fragments to remove the cast after 4 (in case of unreduced fractures) to 5 weeks (after reduction). ${ }^{2}$ The clinical outcome of the fracture healing process, however, is not always good and may include persisting pain, stiffness and weakness of the wrist. ${ }^{3,4}$ As patient factors, injury patterns and fracture-related characteristics play only a limited role in predicting outcome after a distal radius fracture ${ }^{5-7}$, many contributing factors to a poor outcome are still unknown.

In regular daily practice, evaluation of fracture healing is based on clinical examination by the physician in combination with the interpretation of twodimensional (2D) anteroposterior (AP) and lateral radiographs. These evaluations, however, do not provide detailed information with respect to the consolidation of the bone's cortical and trabecular micro-architecture, or the restoration of bone biomechanical competence. Until recently, in vivo assessment of the microarchitecture of bone was not possible in patients due to the lack of accurate measurement techniques and/or technical or radiation issues in vivo.

With the introduction of a new low-dose high-resolution peripheral quantitative computed tomography (HRpQCT) technique, the micro-architectural aspects of bone can be studied in normal subjects and patients. ${ }^{8,9}$ Furthermore, the three-dimensional (3D) images that are acquired with HRpQCT can be used for bone strength estimation by micro finite element analysis ( $\mu \mathrm{FEA}){ }^{10}{ }^{11}$ We hypothesized that these techniques can be used to study the fracture healing process in greater detail, and as such may contribute to the understanding of normal fracture healing, bone changes that are related to poor outcome as well as the influence of medication on fracture healing. In this thesis, we studied for the first time the application of HRpQCT to assess the healing process of distal radius fractures. 


\section{Fracture healing}

Fracture healing is a postnatal regenerative process that allows restoration of the damaged bone to its pre-injury structure and biomechanical function. ${ }^{12}$ The results of intense catabolic (inhibition) and anabolic (stimulation) processes in fracture repair are commonly described using a three-stage model. First, immediately after the injury and in parallel to fracture hematoma formation, an inflammatory reaction ensues, triggering the start of the repair process. Second, soft callus is formed by accumulation of chondrocytes, which is later replaced by woven bone by endochondral bone formation, which consolidates the bone fragments, while at the periosteum callus is formed by intramembranous ossification. Third, a long period of bone remodeling takes place in which the structural integrity of the trabecular region and the cortex are remodeled in order to restore the bone's biomechanical properties..$^{13,14}$ Whereas the first two stages are generally completed within 4 to 5 weeks after fracture, the remodeling stage continues for months to years. ${ }^{13} \mathrm{~A}$ schematic overview of the healing process of diaphyseal fractures is given in Figure 1.

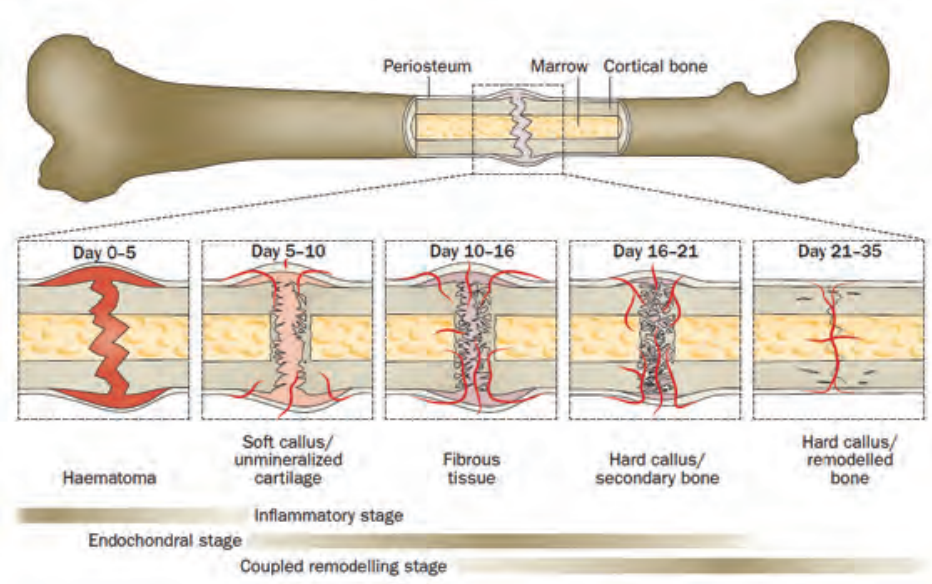

Figure 1 Schematic overview of the different stages during the healing process of diaphyseal fractures. After an initial inflammatory stage in which a hematoma is formed, soft callus is replaced during the endochondral stage by hard callus, which is then remodeled during the remodeling stage. (Adapted with permission from Macmillen Publishers Ltd: Einhorn et al., copyright 2015.)

For metaphyseal fractures, e.g. distal radius fractures, the repair process is slightly different from diaphyseal fracture healing described above due to the presence of trabecular bone which, in contrast to the cortex, is more easily accessible for cells, nutrients and growth factors. As opposed to diaphyseal fractures, the healing process of metaphyseal fractures is thought to be initiated by cells in the midst of the marrow instead of periosteal cells ${ }^{15}$; the role of endochondral bone formation 
is limited, thus producing little external callus ${ }^{16,17}$; and new woven bone is formed directly on and in between existing trabeculae by intramembranous bone formation (Figure 2). $15,17,18$

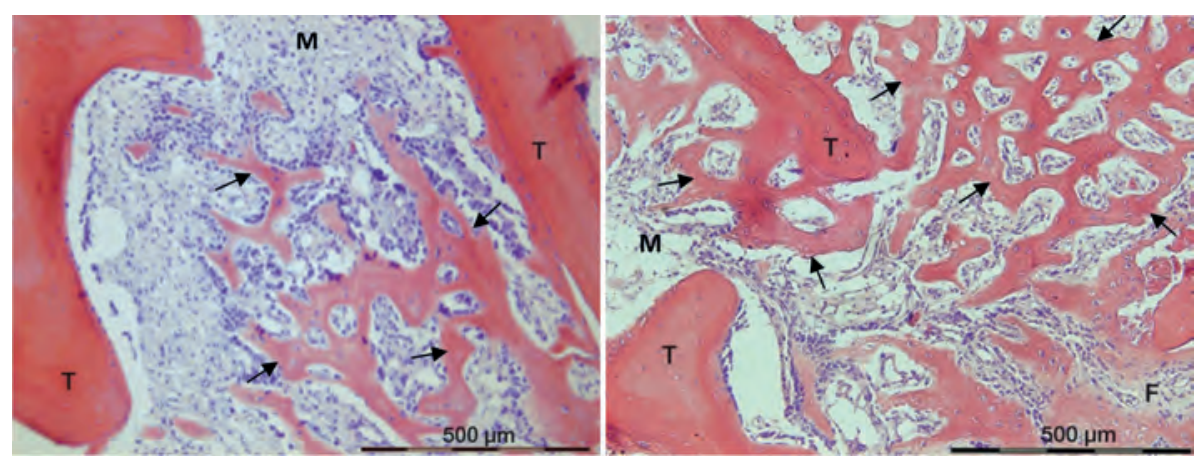

Figure 2 Formation of woven bone (arrows) in the marrow cavity $(M)$ between two old trabeculae $(T)$ (left panel), and woven bone connecting the old trabeculae (right panel) in a distal radius fracture at 16 days post-fracture. Woven bone is still being formed (F) at the lower right-hand corner (right panel). (Reproduced from Aspenberg and Sandberg 2013.)

\section{Evaluation of fracture healing}

In clinical practice, the evaluation of fracture healing is based on clinical findings and interpretation of conventional radiography. Such findings are subjective, and their validity in quantifying fracture healing and bone strength has therefore been questioned. ${ }^{19-21}$ Radiographic evidence of callus formation is dependent on the bone involved and the type and environment of the fracture. ${ }^{22}$ As described, cortical bone fractures start healing at the periosteal and endosteal sites, producing plenty of callus (Figure 1). Metaphyseal fractures, such as stable distal radius fractures, on the other hand, tend to heal endosteally, with new bone formation at the surface of and in between existing trabeculae (Figure 2), and less callus formation and less perceptible cortical bridging. ${ }^{16,23}$ With the introduction of conventional quantitative computed tomography (QCT), volumetric evaluation of bone mineral density (VBMD) and improved three-dimensional (3D) evaluation of fractures became available. ${ }^{24-26}$ However, compared to conventional radiography, clinical CT was not substantially better at determining the repair of distal radius fractures. ${ }^{27}$

In addition, for research purposes, evaluation of the outcome of distal radius fractures is usually assessed using validated questionnaires, such as the patientrated wrist evaluation (PRWE). ${ }^{28,29}$ By the Association of Surgeons of the Netherlands (Nederlandse Vereniging voor Heelkunde, NVvH), the PRWE score is considered as a more important outcome than range of motion or radiological findings. ${ }^{2}$ 


\section{High-Resolution peripheral Quantitative Computed Tomography}

Recently, high-resolution peripheral QCT (HRpQCT) has been developed. HRpQCT is a $3 \mathrm{D}$ quantitative radiographic imaging technique that allows in vivo measurement of volumetric BMD (vBMD) and micro-architecture at the peripheral parts of the body, such as the forearm and lower leg.9, 30 The main advantage of HRpQCT over clinical CT is its higher resolution. Whereas clinical CT has a nominal resolution of approximately $1 \mathrm{~mm}$, HRpQCT has a nominal resolution of $82 \mathrm{~mm}(0.082 \mathrm{~mm})$. Thanks to this high resolution, HRpQCT is able to capture smaller details, such as the individual trabeculae and thin cortices, which is not possible with clinical QCT. ${ }^{31}$ Another advantage of HRpQCT is that scans are made with a low effective dose of $3 \mu \mathrm{Sv}$. Although improvements have recently been made, current disadvantages of HRpQCT in comparison to clinical CT are the limited number of HRpQCT scanners around the world, the longer scan time of 2-3 minutes per $9 \mathrm{~mm}$, and the smaller field of view of $12-14 \mathrm{~cm}$, thus limiting the technique to peripheral parts of the body.

Currently, the only commercially available HRpQCT scanner is the XtremeCT (Scanco Medical A.G. Brütisellen, Switzerland). A standard protocol provided by the manufacturer is available for image acquisition and reconstruction, definition of the region of interest (ROI), extraction of the calcified tissue, i.e. bone, and quantification of vBMD and micro-architecture.

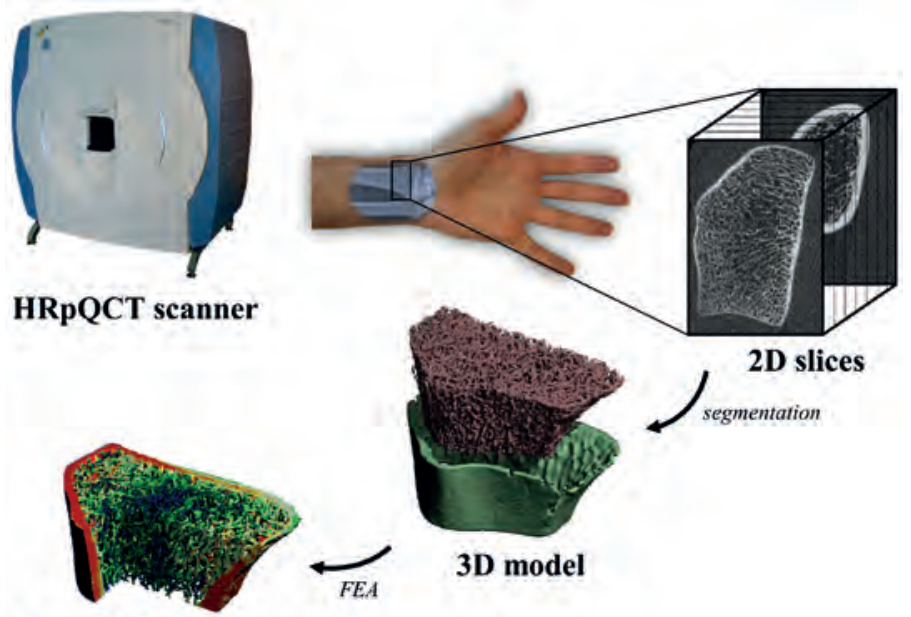

\section{Stress distribution}

Figure 3 Distal radius scanned by HRpQCT (XtremeCT1, Scanco Medical A.G.). A 9 mm long stack of 110 consecutive 2D slices is acquired at the distal radius, which can be converted into a 3D model to measure cortical (green) and trabecular (red) micro-architecture. In addition, the 3D model can be used in finite element analysis (FEA) to estimate bone strength. 


\section{Finite Element Analysis}

Finite element analysis (FEA) is a technique that has its origin in the field of civil engineering. By dividing a 3D model of a structure, such as a bridge, into smaller elements, and subsequently assigning material properties to these elements, and imposing appropriate boundary conditions onto the model, mechanical properties of the object can be estimated. The same principle can also be applied onto bone, where a representative 3D model of the bone's micro-architecture can be created by converting each bone voxel of the segmented 3D model into a brick element of the same size (Figure 3). ${ }^{32}$ Using this approach, Pistoia et al. showed that failure load (strength) of bone specimens can be accurately estimated by micro-FEA ( $\mu$ FEA) based on high-resolution CT scans. ${ }^{33}$ Although constant material properties are used in the $\mu$ FEA that is implemented in the standard workflow on the XtremeCT ${ }^{33}$, they can also vary with voxel intensity. ${ }^{10}$

\section{Objectives}

Since plain radiography, which is clinically the gold standard to evaluate fracture healing of stable distal radius fractures, cannot capture micro-architectural changes and quantify the restoration of bone strength, we hypothesized that a better understanding of distal radius fracture healing might be gained by using HRpQCT and $\mu$ FEA. Although HRpQCT and $\mu$ FEA have been used to study bone quality during aging ${ }^{11}$, with different disorders ${ }^{34}$, or after medication ${ }^{35,36}$, they have never been applied in a clinical setting to study the repair of fractures. The aim of this thesis, therefore, is to explore the application of HRpQCT in combination with $\mu F E A$ to longitudinally study the healing process of stable distal radius fractures.

\section{Outline}

This thesis consists of three parts. In the first part (Chapter 2-4), the influence of different plaster materials on HRpQCT parameters (Chapter 2) and the healing process of distal radius fractures as assessed by HRpQCT during the first twelve weeks (Chapter 3 and 4) is studied. Since casts are often used in the treatment of stable distal radius fractures, this could affect the reproducibility of the HRpQCT measurements and/or introduce an error in the bone parameters that are derived from the HRpQCT images. The effect of a plaster-of-Paris and a fiberglass cast is described in Chapter 2. Next, the healing process of distal radius fractures as 
assessed by HRpQCT is described for the first twelve weeks after fracture. First, in Chapter 3, the changes that occur at the fracture site are described qualitatively using the HRpQCT images as well as quantitatively by studying the vBMD, microarchitecture and bone biomechanical parameters during the healing process. Because more detailed 3D information regarding the bone healing process is obtained than with $2 \mathrm{D}$ radiographs, we hypothesized that (changes in) these parameters might predict clinical outcome, such as wrist pain and function. Therefore, the relation between early changes in the bone parameters and the PRWE score at twelve weeks post-fracture is studied in Chapter 4.

The second part (Chapter 5 and 6) comprises a follow-up of the first part in order to study the long-term changes at both the fractured and non-fractured side. In Chapter 5, vBMD, micro-architectural parameters and biomechanical competence at the fracture side were compared to the non-fractured side at two years postfracture. Additionally, in Chapter 6, the two-year changes in vBMD, microarchitecture and biomechanical parameters at the non-fractured side following a distal radius fracture are studied.

In the third and last part (Chapter 7 and 8), two methods that can be used to extend the applicability of HRpQCT to accurately study the healing process of unstable distal radius fractures are described. For accurate longitudinal analysis of bone parameters, it is important that the same region is analyzed. In unstable fractures, fragments may move relative to each other, and this displacement needs to be corrected in order to analyze the same region. A method for overlaying two consecutive HRpQCT scans of a healing fracture is tested in Chapter 7. Since internal fixation by distal radius plates is the recommended treatment for unstable distal radius fractures, and these plates are generally made from titanium, which leads to image artifacts, plates made from metal-artifact-free material are required for reliable assessment of bone parameters. In Chapter 8, we therefore assessed the effect of a volar distal radius plate made from carbon-fiber-reinforced plastic on the bone parameters obtained from HRpQCT images in comparison to titanium plates.

Finally, Chapter 9 comprises a discussion of the main findings of the previous chapters, addresses the main limitations of the studies, provides final conclusions and recommendations, and includes opportunities for future research. 


\section{References}

1. de Putter, C.E. et al. Epidemiology and health-care utilisation of wrist fractures in older adults in The Netherlands, 1997-2009. Injury 44, 421-6 (2013).

2. The Association of Surgeons of the Netherlands. Guideline Distal Radius Fractures: Diagnosis and Treatment (Dutch) (Utrecht, 2010).

3. MacDermid, J.C., Roth, J.H. \& Richards, R.S. Pain and disability reported in the year following a distal radius fracture: a cohort study. BMC Musculoskelet Disord 4, 24 (2003).

4. Bialocerkowski, A.E. Difficulties associated with wrist disorders--a qualitative study. Clin Rehabil 16, 429-40 (2002).

5. MacDermid, J.C., Donner, A., Richards, R.S. \& Roth, J.H. Patient versus injury factors as predictors of pain and disability six months after a distal radius fracture. J Clin Epidemiol 55, 849-54 (2002).

6. Grewal, R., MacDermid, J.C., Pope, J. \& Chesworth, B.M. Baseline predictors of pain and disability one year following extraarticular distal radius fractures. Hand (N Y) 2, 104-11 (2007).

7. Karnezis, I.A. \& Fragkiadakis, E.G. Association between objective clinical variables and patient-rated disability of the wrist. J Bone Joint Surg Br 84, 967-70 (2002).

8. Boutroy, S., Bouxsein, M.L., Munoz, F. \& Delmas, P.D. In vivo assessment of trabecular bone micro-architecture by high-resolution peripheral quantitative computed tomography. J Clin Endocrinol Metab 90, 6508-15 (2005).

9. MacNeil, J.A. \& Boyd, S.K. Accuracy of high-resolution peripheral quantitative computed tomography for measurement of bone quality. Med Eng Phys 29, 1096-105 (2007).

10. Macneil, J.A. \& Boyd, S.K. Bone strength at the distal radius can be estimated from highresolution peripheral quantitative computed tomography and the finite element method. Bone 42, 1203-13 (2008).

11. Dalzell, N. et al. Bone micro-architecture and determinants of strength in the radius and tibia: age-related changes in a populationbased study of normal adults measured with high-resolution pQCT. Osteoporos Int 20, 1683-94 (2009)

12. Einhorn, T.A. \& Gerstenfeld, L.C. Fracture healing: mechanisms and interventions. Nat Rev Rheumatol 11, 45-54 (2015).
13. Marsell, R. \& Einhorn, T.A. The biology of fracture healing. Injury 42, 551-5 (2011).

14. Pivonka, P. \& Dunstan, C.R. Role of mathematical modeling in bone fracture healing. Bonekey Rep 1, 221 (2012).

15. Aspenberg, P. \& Sandberg, O. Distal radial fractures heal by direct woven bone formation. Acta Orthop 84, 297-300 (2013).

16. Uhthoff, H.K. \& Rahn, B.A. Healing patterns of metaphyseal fractures. Clin Orthop Relat Res, 295-303 (1981).

17. Chen, W.T. et al. A special healing pattern in stable metaphyseal fractures. Acta Orthop 86, 238-42 (2015).

18. Jarry, L. \& Uhthoff, H.K. Differences in healing of metaphyseal and diaphyseal fractures. Can J Surg 14, 127-35 (1971).

19. Blokhuis, T.J. et al. The reliability of plain radiography in experimental fracture healing. Skeletal Radiol 30, 151-6 (2001).

20. Panjabi, M.M., Lindsey, R.W., Walter, S.D. \& White, A.A., 3rd. The clinician's ability to evaluate the strength of healing fractures from plain radiographs. J Orthop Trauma 3 29-32 (1989).

21. Davis, B.J. et al. Reliability of radiographs in defining union of internally fixed fractures. Injury 35, 557-61 (2004).

22. Marsh, D. Concepts of fracture union, delayed union, and nonunion. Clin Orthop Relat Res, S22-30 (1998).

23. Sevitt, $S$. The healing of fractures of the lower end of the radius. A histological and angiographic study. J Bone Joint Surg Br 53, 519-31 (1971).

24. Bhattacharyya, T. et al. The accuracy of computed tomography for the diagnosis of tibial nonunion. J Bone Joint Surg Am $\mathbf{8 8}$ 692-7 (2006).

25. Singh, H.P. et al. Partial union of acute scaphoid fractures. J Hand Surg Br 30, 440-5 (2005).

26. Zhu, Y. et al. Computed tomography-based Three-Column Classification in tibial plateau fractures: introduction of its utility and assessment of its reproducibility. J Trauma Acute Care Surg 73, 731-7 (2012).

27. Grigoryan, M. et al. Quantitative and qualitative assessment of closed fracture healing using computed tomography and conventional radiography. Acad Radiol 10, 1267-73 (2003).

28. MacDermid, J.C. Development of a scale for patient rating of wrist pain and disability. $J$ Hand Ther 9, 178-83 (1996). 
29. Brink, S.M., Voskamp, E.G., Houpt, P. \& Emmelot, C.H. Psychometric properties of the Patient Rated Wrist/Hand Evaluation Dutch Language Version (PRWH/E-DLV). J Hand Surg Eur Vol 34, 556-7 (2009).

30. Boutroy, S., Bouxsein, M.L., Munoz, F. \& Delmas, P.D. In vivo assessment of trabecular bone micro-architecture by high-resolution peripheral quantitative computed tomography. The Journal of clinical endocrinology and metabolism 90, 6508-15 (2005).

31. Prevrhal, S., Engelke, K. \& Kalender, W.A. Accuracy limits for the determination of cortical width and density: the influence of object size and CT imaging parameters. Phys Med Biol 44, 751-764 (1999).

32. van Rietbergen, B., Weinans, H., Huiskes, R. \& Odgaard, A. A new method to determine trabecular bone elastic properties and loading using micromechanical finiteelement models. J Biomech 28, 69-81 (1995).
33. Pistoia, W. et al. Estimation of distal radius failure load with micro-finite element analysis models based on three-dimensional peripheral quantitative computed tomography images. Bone 30, 842-848 (2002).

34. Romme, E.A. et al. Bone stiffness and failure load are related with clinical parameters in men with chronic obstructive pulmonary disease. J Bone Miner Res (2013).

35. Burghardt, A.J. et al. A longitudinal HRPQCT study of alendronate treatment in postmenopausal women with low bone density: Relations among density, cortical and trabecular micro-architecture, biomechanics, and bone turnover. J Bone Miner Res 25, 2558-71 (2010).

36. Chapurlat, R.D. et al. Effect of oral monthly ibandronate on bone micro-architecture in women with osteopenia-a randomized placebo-controlled trial. Osteoporos Int $\mathbf{2 4}$ 311-20 (2013). 


$$
x
$$




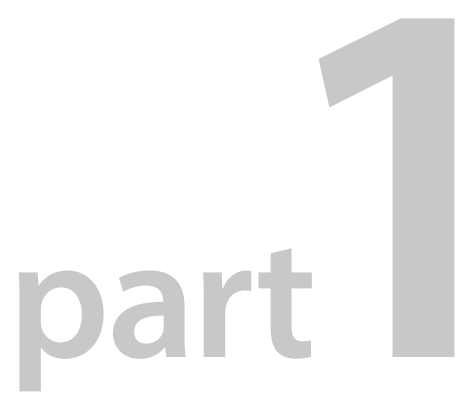

Assessment of stable distal radius fracture healing using $\mathrm{HRpQCT}$ 


$$
\frac{b}{x}
$$




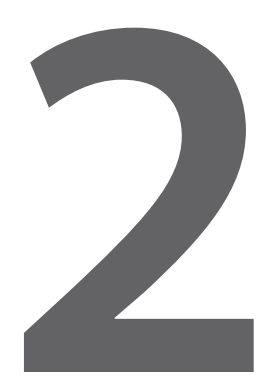

Effect of casting on short-term reproducibility and bone parameters obtained from HRpQCT measurements at the distal radius 


\section{Abstract}

\section{Background}

High-resolution peripheral quantitative computed tomography (HRpQCT) is a promising tool to assess the fracture healing process at the micro-scale in vivo. Since casts are often used during fracture treatment, they might affect the assessment of bone density, micro-architectural and biomechanical parameters and the short-term reproducibility those parameters, e.g. as a result of beam-hardening. The aim of this study was to assess the effect of a plaster-of-Paris and/or fiberglass cast on bone parameters and on the short-term reproducibility of the HRpQCT measurements of those parameters.

\section{Methods}

The effects of a cast on HRpQCT-derived bone parameters were evaluated by comparing HRpQCT scans in fifteen human cadaveric distal radial specimens from one male and fourteen females (median age 84 years [range, 62-90 years] at the time of death) in three conditions: without a cast (control), with a 4-mm-thick plaster-of-Paris cast, and with a fiberglass cast. Short-term reproducibility was assessed using duplicate scans of the distal end of the radius in sixteen healthy volunteers without a fracture (median age 26 years (range 22-39 years); nine men) without and with a fiberglass cast.

\section{Results}

Compared with measurements made with no cast, the plaster-of-Paris cast introduced a systematic error in the bone parameters ranging from $-2.6 \%$ in trabecular separation to $-9.8 \%$ in cortical thickness. Bone parameters were affected only marginally by fiberglass, with errors between -0.6 to $-1.6 \%$ in trabecular separation and cortical thickness, respectively. Short-term reproducibility with a fiberglass cast was similar to that with no cast: approximately $1 \%$ for bone density parameters, 4 to $5 \%$ for microarchitectural parameters and 3 to $4 \%$ for the biomechanical parameters.

\section{Conclusion}

A plaster-of-Paris cast has a considerable effect on HRpQCT measurements. A fiberglass cast only marginally affects the bone parameters, and the short-term reproducibility of HRpQCT measurements with a fiberglass cast is comparable with that in patients without a cast. In studies on fracture healing using HRpQCT, a fiberglass cast is desirable if immobilization is indicated. The use of a plaster-of-Paris cast should be avoided if possible; however, if not avoidable, corrections after the scan are desirable to adjust for the error introduced in the bone parameters. 


\section{Introduction}

Distal radius fractures are among the most common types of fractures. Women are particularly vulnerable, with a lifetime risk of a distal radius fracture reported to range between $13 \%$ and $21 \%$ at 50 years of age. ${ }^{1}$ In closed, non-displaced or reduced stable fractures, cast immobilization is the most common treatment. ${ }^{2}$ At routine follow-up of five to six weeks, when fracture consolidation is expected, usually the cast is removed after clinical and radiographic examination.

For osteoporotic patients, clinicians may question whether this standard fracture treatment is optimal for an individual patient. The influences on the healing process due to osteoporosis ${ }^{3,4}$ or medication ${ }^{5}$ suggest that the treatment of osteoporotic fractures may benefit from patient-specific adjustments, as it may take longer before an osteoporotic fracture can be fully loaded. Thus, it may be warranted to have a more detailed evaluation of the fracture healing process and of bone strength recovery than is possible using only radiographs.

High-resolution peripheral quantitative computed tomography (HRpQCT) is a promising tool to assess the fracture healing process at the micro-scale in vivo. In a recent study, we demonstrated that detailed information about changes in bone density, micro-architecture and bone mechanical properties during the healing of stable distal radius fractures can be obtained using high-resolution peripheral quantitative computed tomography (HRpQCT). ${ }^{6,7}$ Because that study was the first in which HRpQCT was used to monitor fracture healing, the accuracy (i.e. closeness to the true value) of the obtained bone parameters and the shortterm reproducibility (ability to reproduce similar results, given no real biological change) of the measurements are two important considerations that need to be addressed.

For standard HRpQCT measurements, the accuracy and reproducibility of the bone density, micro-architectural and mechanical parameters have been tested and validated extensively. ${ }^{8-11}$ When studying fracture healing of the distal radius, however, the wrist is usually immobilized with a forearm-based cast. Hence, HRpQCT measurements of the distal radius are performed with a cast; therefore, the cast may adversely influence the obtained bone parameters and the reproducibility of those measurements. First, a cast will absorb some radiation, which could lead to unwanted effects such as beam-hardening and increased noise. Thus, for common cast materials, the results might be affected because of these potential deteriorating effects on image quality. ${ }^{12}$ Second, the presence of a cast precludes the use of the standard holder ${ }^{8}$ and thus requires a nonstandard cylindrical holder to fixate the forearm immobilized in a cast. In that situation, the cast rather than the forearm is fixated, leaving room for potential movement of the 
forearm within the cast. This seemingly less strict fixation could in turn affect the reproducibility of the measurements.

The purpose of this study, therefore, was to quantify the extent to which a cast would affect 1) the bone density, micro-architectural and mechanical parameters obtained from HRpQCT measurements, and 2) the short-term reproducibility of those measurements.

\section{Methods}

\section{Material and subjects}

To measure the effect of a cast on bone parameters obtained from HRpQCT measurements, fifteen human cadaveric radii (from one male and fourteen female donors with a median age 84.0 years [range, 62-90 years] at the time of death) were imaged by HRpQCT in three conditions: without a cast (control), with a 4-mm-thick plaster-of-Paris cast, and with a fiberglass cast (Figure 1). The radii were obtained from the Department of Anatomy and Embryology of the University of Amsterdam and were preserved in formalin. The history of the donors was unknown and no selection criteria were applied.

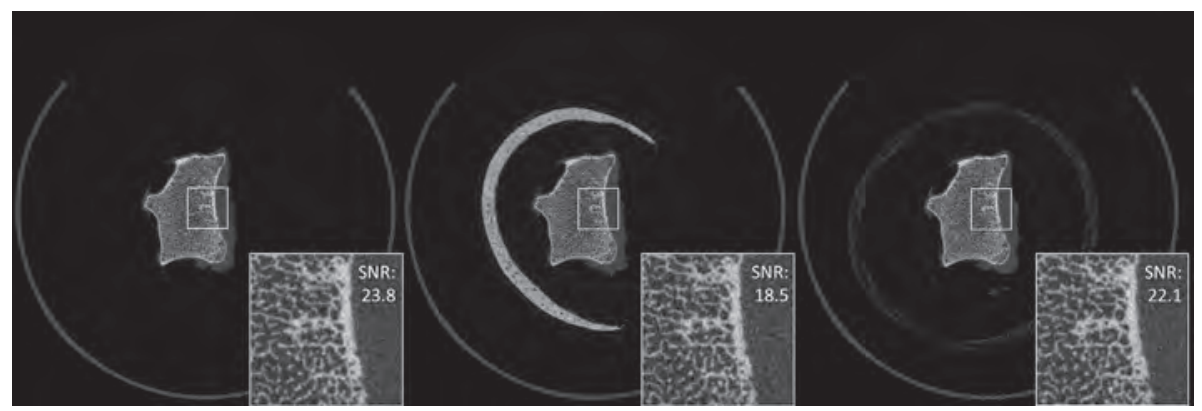

Figure 1 Each cadaveric radius was scanned by HRpQCT in three conditions: without a cast (left), with a plaster-of-Paris cast (middle) and with a fiberglass cast (right). With the plaster-of-Paris cast, the image had a higher noise level with lower bone densities. SNR: signal-to-noise ratio

To compare the short-term reproducibility of HRpQCT measurements without and with a cast, sixteen healthy individuals (nine men and seven women with a median age 25.5 years [range, 22-39 years]) without a fracture underwent four separate HRpQCT scans of the distal radius on the dominant side within four hours: two consecutive HRpQCT scans without a cast and two consecutive HRpQCT scans with a fiberglass cast. The forearm of each participant was completely repositioned between each scan. The casts were applied by a professional cast technician. 
Because the standard positioning holder that is supplied by the manufacturer could not fit the forearm with a cast, we used a newly designed cylindrical holder for all measurements, i.e. CT Carpal X1 (Pearltec AG, Switzerland). This holder, which was also used in our earlier studies ${ }^{6,7}$, consists of a cylindrical carbon fiber tube and an inflatable bag with soft liner on the inside (Figure 2), allowing firm fixation of the forearm.

This feasibility study was performed within the framework of a larger fracture healing study approved by our institutional medical ethics committee (registration number NTR3821). All participants were informed and gave written permission for the scans.

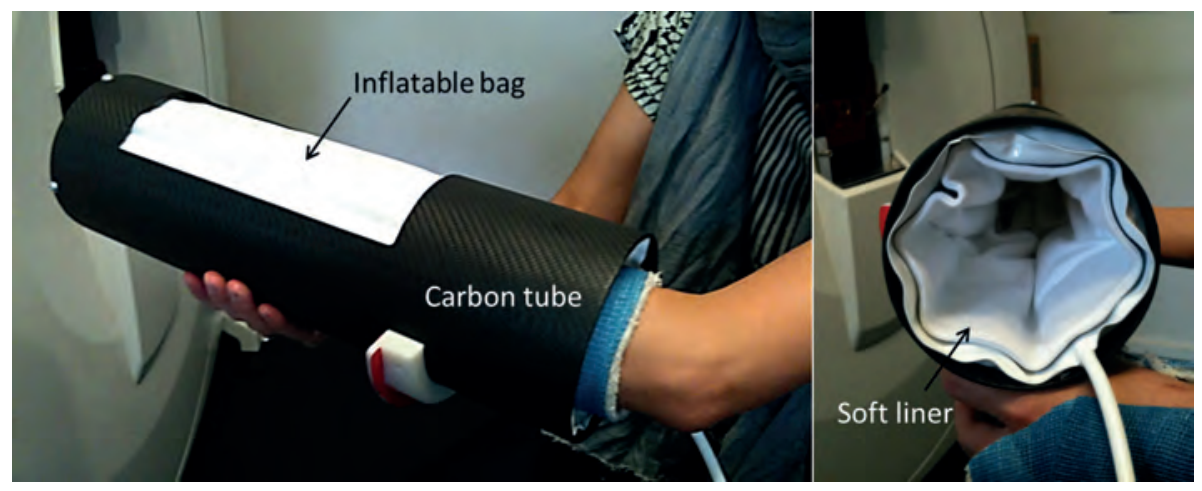

Figure 2 Fixation of the forearm with a cast in the CT Carpal X1 holder. This holder consists of a cylindrical carbon tube and an inflatable bag with soft padding of the inside. (Reproduced with permission of Pearltec AG.)

\section{Scanning by HRPQCT}

All radii were imaged by HRpQCT (XtremeCT, Scanco Medical AG, Switzerland) at clinical in vivo settings recommended by the manufacturer (peak voltage of $60 \mathrm{kVp}$, tube current of $900 \mu \mathrm{A}$, and $100 \mathrm{~ms}$ integration time). A scout view was made before each measurement, to determine the region of interest (ROI). The distal end of the radius proximally from the subchondral plate was chosen as the $\mathrm{ROI}$, because this is the region where a fracture of the radius typically occurs. In the cadaveric radii, the ROI started just below the subchondral plate (Figure 3 ). In the healthy individuals, the ROI started at an offset of $5 \mathrm{~mm}$ from the distal edge of the lunate bone (Figure 3). Because some distal radius fractures might not be fully covered by one standard stack of 110 slices, the scan length was set to $18.04 \mathrm{~mm}$ for all measurements. With an isotropic voxel size of $82 \mathrm{~mm}$, each measurement resulted in 220 parallel CT slices. The total effective radiation dose received per individual was $24 \mathrm{mSv}$. 
After scanning, all HRpQCT images were checked by one operator (J.J.A.d.J.) for motion-induced image artifacts and were quality-graded as described by Pialat et al. $^{13}$ This grading system consists of five grades (ranging from 1 , indicating no motion artifacts, to 5, indicating extreme motion artifacts), where grade 1, 2 and 3 are considered to be of sufficient quality because of cortical continuity. ${ }^{13}$
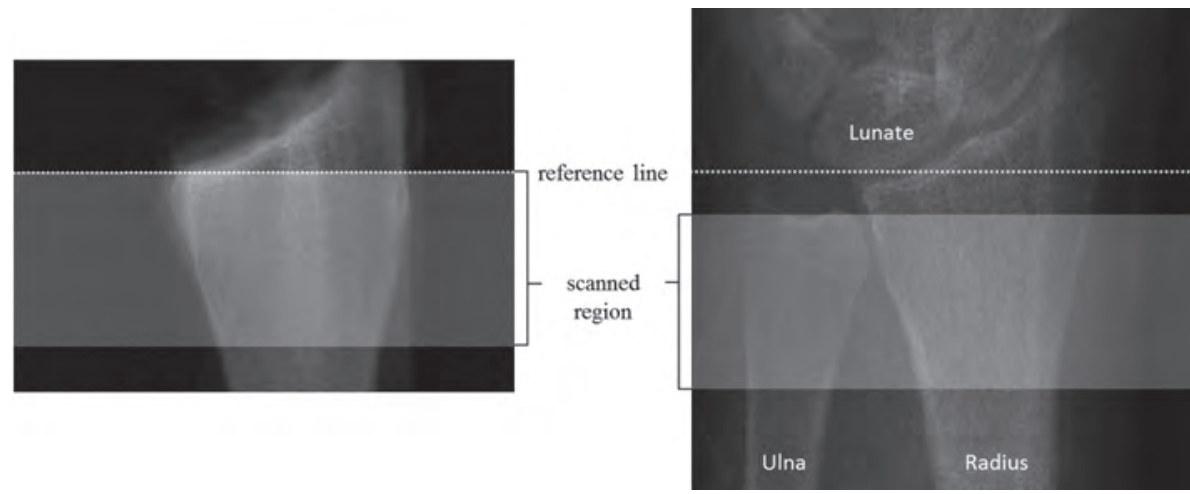

Figure 3 Regions of interest scanned by HRpQCT in the cadaveric radii (left) and in the healthy individuals (right). With a scan length of $18.04 \mathrm{~mm}$, each scan resulted in 220 slices.

\section{Evaluation of bone density and micro-architecture}

The HRpQCT images were evaluated using the standard patient evaluation protocol that was provided by the manufacturer and that has been described previously in detail. ${ }^{14,15,16}$ The following bone density parameters were calculated from the images: total, trabecular and cortical volumetric bone mineral density (vBMD). Also bone volume to total volume (BV/TV) was calculated for the trabecular region. The micro-architectural parameters that were calculated in the trabecular region were the trabecular number, thickness and separation. For the cortical region, the cortical thickness was obtained.

\section{$\mu F E$ analysis}

Micro-finite element ( $\mu \mathrm{FE}$ ) models were created directly from the segmented HRpQCT images, similar to earlier studies. ${ }^{17,18}$ In short, each voxel that represents bone tissue was converted into a brick element of the same size, thus creating a representative $\mu \mathrm{FE}$ model of the micro-architecture of the bone. Equal properties were assigned to every element: a Young's modulus of $10 \mathrm{GPa}$ and a Poisson's ratio of 0.3 . By applying a 'high friction' compression test in the axial direction as described by Pistoia et al. ${ }^{17}$,compression stiffness and estimated ultimate load were determined. ${ }^{17}$ 


\section{Statistical analysis}

Deviation in the bone parameters obtained from the HRpQCT scans made with the plaster-of-Paris and fiberglass cast was reported as the percent difference relative to the measurement without a cast. A repeatedmeasures design was used to compare the effect of both casts on the bone parameters. If the Fratio was significant, a paired ttest with Bonferroni correction was applied to assess whether the difference, compared to the condition without a cast, was significant.

Short-term reproducibility without and with a fiberglass cast was calculated using the duplicate in vivo measurements per condition and was reported as the root-mean-square coefficient of variation (RMS-CV) as described by Gluer et al. ${ }^{19}$ Wilcoxon signed-rank testing was used to test whether there was a difference in short-term reproducibility of the bone parameters between the conditions with and without a cast.

A significance level of 0.05 was used for all tests. All statistical analyses were performed with SPSS Statistics for Windows version 20.0 (IBM Corp., Armonk, USA).

\section{Results}

\section{Errors in bone parameters}

The mean bone density, micro-architectural and mechanical parameters (and standard deviation) at the cadaveric radii measured without a cast (control), with a 4-mm plaster-of-Paris cast, and with a fiberglass cast are presented in Table 1. All bone parameters were affected by the presence of a cast (the Fratio for all bone parameters was significant; $p<0.001$ ).

The magnitude of the errors in the measurements with a fiberglass cast ranged from 0.6 to $1.6 \%$. The errors in the bone density and mechanical parameters ranged between -0.9 and $-1.1 \%$, indicating a slight underestimation of the (cast-immobilized) bone density. Trabecular and cortical thickness were also underestimated, by -1.5 and $-1.6 \%$, respectively. Trabecular number was overestimated by $0.7 \%$ and trabecular separation was underestimated by $-0.6 \%$.

For the plaster-of-Paris cast measurements, the errors were larger for all bone parameters, ranging in magnitude from 2.6 to $9.8 \%$. Again, an underestimation was found for bone density, thickness and biomechanical parameters, whereas there was an overestimation for trabecular number. For the bone density parameters, the errors ranged from -3.4 to $-5.3 \%$. For trabecular and cortical thickness, the errors were -6.5 and $-9.8 \%$, and the errors in compression stiffness and estimated ultimate failure load were -7.5 and $-6.6 \%$. The error in trabecular number was 


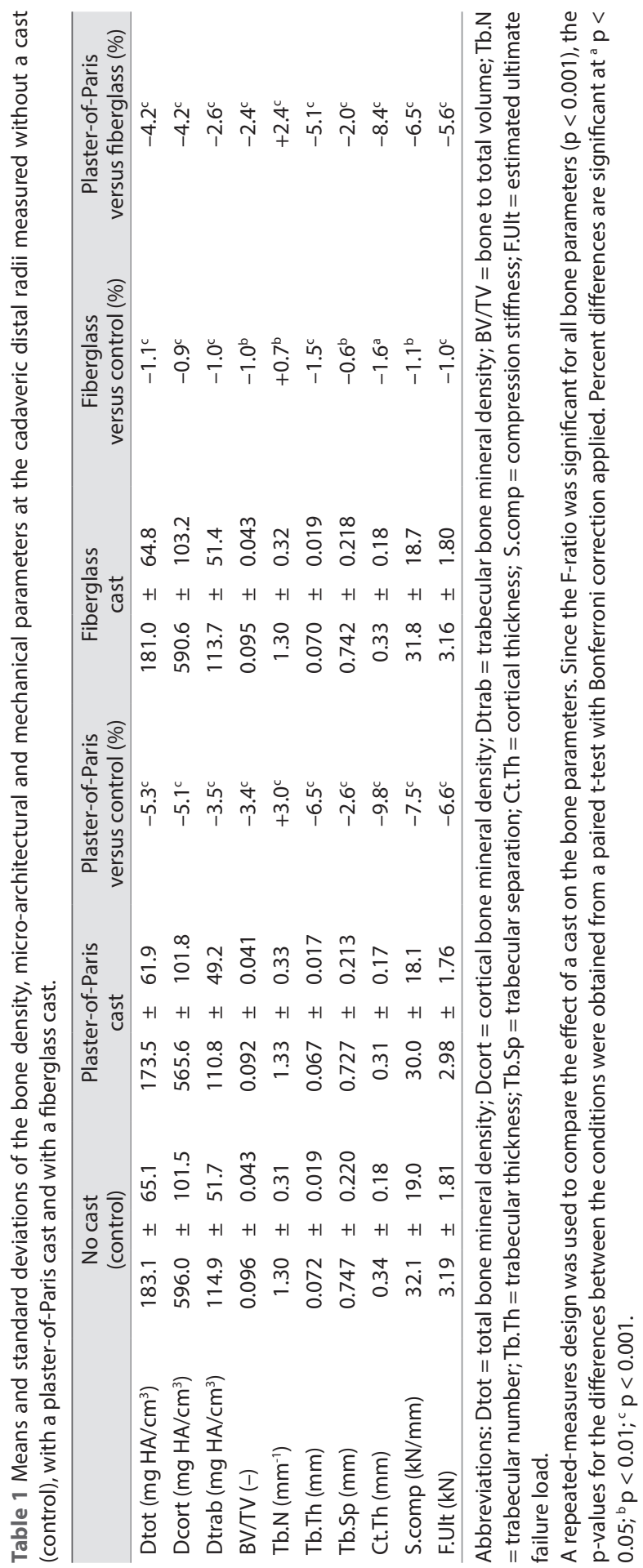


$3.0 \%$. The smallest error in the plaster cast measurements was $-2.6 \%$ for trabecular separation.

\section{Short-term reproducibility}

Characteristics of the healthy individuals and the bone parameters measured at the distal radius without a cast are listed in Table 2. From the 64 initial measurements, seven measurements (11\%), six done without a cast and one with a cast, were of insufficient image quality because of motion artifacts. From the 32 measurements without a cast, eleven scans were grade 1 ; nine scans grade 2 ; six scans grade 3 , five scans grade 4 ; and one scan grade 5 . The grade- 5 scan contained a large amount of extreme motion artifacts, because the participant tried to remove his forearm from the positioning holder during scanning. Therefore, this scan was excluded from short-term reproducibility calculation. From the 32 measurements with a cast, eight scans were grade 1 ; thirteen scans grade 2 ; ten scans grade 3 ; and one scan grade 4.

Table 2 Characteristics of the sixteen healthy individuals and the bone density, micro-architectural, and biomechanical parameters [mean (standard deviation)] measured at the distal end of the radius without a cast

\begin{tabular}{lc}
\hline Variable & Finding \\
\hline Sex $(\mathrm{M} / \mathrm{F})$ & $9 / 7$ \\
$\mathrm{Age}^{*}($ years $)$ & $25.5(7.0)$ \\
$\mathrm{BMI}\left(\mathrm{kg} / \mathrm{m}^{2}\right)$ & $23.5(2.5)$ \\
Dtot $\left(\mathrm{mgHA} / \mathrm{cm}^{3}\right)$ & $332.3(56.8)$ \\
Dcort $\left(\mathrm{mgHA} / \mathrm{cm}^{3}\right)$ & $865.0(37.4)$ \\
Dtrab $\left(\mathrm{mgHA} / \mathrm{cm}^{3}\right)$ & $171.7(51.1)$ \\
BV/TV $(-)$ & $0.143(0.043)$ \\
Tb.N $\left(\mathrm{mm}^{-1}\right)$ & $1.96(0.33)$ \\
Tb.Th $(\mathrm{mm})$ & $0.072(0.012)$ \\
Tb.Sp $(\mathrm{mm})$ & $0.451(0.094)$ \\
Ct.Th $(\mathrm{mm})$ & $0.86(0.16)$ \\
S.comp $(\mathrm{kN} / \mathrm{mm})$ & $47.1(11.0)$ \\
F.Ult $(\mathrm{kN})$ & $4.64(1.08)$ \\
\hline
\end{tabular}

* Reported as median and interquartile range. Abbreviations: $\mathrm{BMI}=$ body mass index; $\mathrm{Dtot}=$ total bone mineral density; Dcort = cortical bone mineral density; Dtrab = trabecular bone mineral density; BV/ $\mathrm{TV}=$ bone to total volume; Tb. $\mathrm{N}=$ trabecular number; $\mathrm{Tb} . \mathrm{Th}=$ trabecular thickness; $\mathrm{Tb} . \mathrm{Sp}=$ trabecular separation; Ct.Th = cortical thickness; S.comp = compression stiffness ; F.Ult = estimated ultimate failure load.

RMS-CVs for the short-term reproducibility without wearing a cast were found to be generally between $0.6 \%$ and $4.0 \%$ (Table 3 ). Whereas CVs of density parameters varied between $0.6 \%$ (total BMD) and $1.1 \%$ (cortical BMD), the CVs for structural parameters were higher: $1.7 \%$ for cortical thickness and between 3.5\% (trabecular 
thickness) and $4.0 \%$ (trabecular separation) for the trabecular structural indices. The biomechanical parameters compression stiffness and estimated ultimate failure load showed CVs of 3.3 and 3.7\%, respectively. Short-term reproducibility was not significantly different with a fiberglass cast for any of the bone parameters, except for cortical thickness. In general, the CVs for the bone density parameters were slightly lower when a fiberglass cast was worn (0.5 to $0.7 \%$ ) than when one was not worn (0.6 to $1.1 \%)$. Except for cortical thickness, the CVs for the bone micro-architectural and biomechanical parameters were slightly higher when a fiberglass cast was worn (3.7 to $5.3 \%$ ) than when one was not worn (3.3 to $4.0 \%$ ). However, these differences did not reach statistical significance (Table 3). Only the $\mathrm{CV}$ of cortical thickness was significantly lower when a fiberglass cast was worn ( $p$ $=0.028$ ).

Table 3 Short-term reproducibility (RMS-CV and 95\% confidence intervals) of HRpQCT derived bone parameters measured in vivo without and with the presence of a fiberglass cast.

\begin{tabular}{|c|c|c|c|}
\hline & $\begin{array}{l}\text { Without fiberglass cast } \\
\qquad(n=15) \\
\text { RMS-CV }(\%)\end{array}$ & $\begin{array}{l}\text { With fiberglass cast } \\
\qquad(n=16)\end{array}$ & n-value \\
\hline & & & \\
\hline Dtot & 1.10 (0.60 to 2.64$)$ & 0.66 (0.37 to 1.54$)$ & 0.061 \\
\hline Dcort & 0.60 (0.33 to 1.45$)$ & $0.47(0.26$ to 1.09$)$ & 0.191 \\
\hline Dtrab & 1.01 (0.55 to 2.43 ) & 0.64 (0.35 to 1.48$)$ & 0.100 \\
\hline $\mathrm{BV} / \mathrm{TV}$ & 1.12 (0.61 to 2.68$)$ & $0.68(0.38$ to 1.58$)$ & 0.062 \\
\hline Tb.N & 3.84 (2.09 to 9.18$)$ & $5.30(2.94$ to 12.3$)$ & 0.776 \\
\hline Tb.Th & 3.52 (1.92 to 8.41$)$ & 5.13 (2.84 to 11.9$)$ & 0.347 \\
\hline Tb.Sp & 3.95 (2.16 to 9.46$)$ & 5.31 (2.94 to 12.3$)$ & 0.955 \\
\hline Ct.Th & 1.66 (0.90 to 3.96$)$ & 0.90 (0.50 to 2.10$)$ & 0.028 \\
\hline S.comp & 3.33 (1.82 to 7.97$)$ & $3.72(2.06$ to 8.60$)$ & 0.756 \\
\hline F.Ult & 3.72 (2.03 to 8.90$)$ & 4.36 (2.42 to 10.1$)$ & 0.877 \\
\hline
\end{tabular}

Abbreviations: Dtot $=$ total bone mineral density; Dtrab $=$ trabecular bone mineral density; Dcort = cortical bone mineral density; BV/TV = bone to total volume; $\mathrm{Tb} . \mathrm{N}=$ trabecular number; $\mathrm{Tb} . \mathrm{Th}$ $=$ trabecular thickness; Tb.Sp $=$ trabecular separation; $\mathrm{Ct} . \mathrm{Th}=$ cortical thickness; $\mathrm{S} . \mathrm{Comp}=$ compression stiffness ; F.Ult $=$ estimated ultimate failure load. P-values were obtained from a Wilcoxon signed-rank test.

\section{Discussion}

The present study evaluated the effect of a cast on bone density, micro-architectural and biomechanical parameters obtained from HRpQCT measurements at the distal end of the radius, and the short-term reproducibility of those parameters.

\section{Errors in bone parameters}

The results suggest that both types of casts introduced systematic errors in the bone parameters. However, the differences when a fiberglass cast was worn and when 
no cast was worn were very small (approximately 1\%). Although significant, those differences may not be clinically relevant, in particular when the reproducibility error and the fact that changes during fracture healing are generally in the range of $10 \%$ to $20 \%$ are considered. ${ }^{6}$

The use of plaster of Paris, on the other hand, can considerably bias measurement results. Compared with scanning without a cast, many parameters deviated by $5 \%$ to almost $10 \%$. Admittedly, such a bias might be acceptable in follow-up studies that aim at quantifying changes in parameters over time. Additionally, depending on whether a plaster-of-Paris cast is in place or removed, it presumably would be possible to correct the calculated bone parameters according to the percentages presented in Table 1. However, these values were obtained in cadaveric radii, with low bone density (because of old age), that were not scanned at the standard region. Also, we did not vary the thickness of the plaster-of-Paris cast. Since the thickness of plaster-of-Paris casts might differ between patients, the errors in the bone parameters caused by the plaster-ofParis casts might vary as well. Hence the presently reported percentages are not immediately applicable to the general patient population in the clinical setting and should at least be adjusted proportionally to the thickness of the casts used on patients.

The difference between the effect caused by a fiberglass cast and a plasterof-Paris cast can be explained by the different material properties of the two casts (see Appendix). At the standard settings of the XtremeCT, plaster of Paris absorbs more lower-energy photons than fiberglass does $^{20}$, resulting in underestimation of the bone density and increased beam-hardening artifacts and noise (Figure 1). These effects were confirmed by phantom measurements with and without a plaster-of-Paris cast (results not shown). Due to the beam-hardening effect and the increased noise, segmentation of the trabecular structure apparently results in a 3D model with more, but thinner, trabeculae and a lower estimated bone stiffness and strength.

\section{Short-term reproducibility}

We found that when HRpQCT and the non-standard cylindrical holder (without a cast) were used, bone density parameters can be assessed with high precision (RMS-CVs of $0.6 \%$ to $1.1 \%$ ) and micro-architecture and biomechanical parameters with good precision (RMS-CVs of $1.7 \%$ to $4.0 \%$ ). Similar values have been reported in literature using the standard holder and no cast.8, 9 Surprisingly, short-term reproducibility improved slightly when the wrist was immobilized by a cast. Presumably, the cast leads to a better fixation of the forearm in the cylindrical holder, resulting in fewer motion artifacts. 
It should be noted that the reproducibility measured in this study was obtained from HRpQCT measurements that require twice as much scanning time due to increased scan length compared to standard HRpQCT measurements, i.e. 5.6 minutes and $18 \mathrm{~mm}$ versus 2.8 minutes and $9 \mathrm{~mm}$, respectively. As with longer scan times, the chance of motion-induced artifacts increases, and an increased scan time in principle could negatively affect the reproducibility. However, the increased scan time in our study did not decrease the short-term reproducibility of the measurements. A possible explanation for this is that the cylindrical holder with its inflatable bag better fixated the forearm than the standard holder used in other studies.

For the clinical application of HRpQCT to monitor fracture healing, it is important to know the precision of the HRpQCT measurements (with a cast), since this determines the change in bone parameters that can be considered significant (expressed as the least significant change (LSC).21 Given the measured short-term reproducibility in this study and a statistical confidence level of $95 \%$, the LSC for the bone density parameters is approximately $2 \%$. The LSC for the micro-architectural parameters the was $14 \%$ and $11 \%$ for the biomechanical parameters. When these LSC values were compared with the changes that we found in our previously reported study on fracture healing ${ }^{6}$, we notice that the observed changes in bone parameters are well above these LSCs. For example, cortical and trabecular bone density changed $-4.1 \%$ and $20 \%$, respectively, at six to eight weeks post-fracture. For the structural parameters, trabecular thickness changed by $30 \%$ at twelve weeks post-fracture, and at the same time, biomechanical parameters increased by $31 \% .{ }^{6}$ Hence, the precision of HRpQCT measurements with a cast is good enough to reliably detect changes in bone parameters typically occurring during the fracture healing process.

\section{Limitations}

Reproducibility of a measurement is ideally assessed in a group of subjects similar to the group in which the measurement will be performed, in this instance osteoporotic women over 50 years of age with a distal radius fracture. As mentioned before, we used healthy individuals between 22 and 39 years old. Nevertheless, recent data have indicated that differences in reproducibility between age groups are small, and that reproducibility does not necessarily decrease with age. ${ }^{22}$

Another limitation is the modest number of individuals in the reproducibility group, and hence, the large confidence intervals of the RMS-CV.

In addition, the reproducibility study was performed with a fiberglass cast only and not a plaster-of-Paris cast. However, it seems unlikely that motion artifacts, which are the major source of reproducibility errors, would differ between the two cast materials. 
Last, the cadaveric radii were scanned without soft tissue surrounding the bone and in the absence of the ulna. Although we did use a repeated-measures design, this extra tissue would have absorbed some of the radiation and the results might be different in intact forearms.

\section{Conclusion}

In conclusion, bone parameters obtained from HRpQCT measurements at the distal radius and the short-term reproducibility of these measurements are only marginally affected by a fiberglass cast. However, a considerable bias in the measurement results is to be expected when a plaster-of-Paris cast is used so corrections ideally should be made after scanning. On the basis of these results, we recommend immobilization with a fiberglass cast if possible when monitoring distal radius fracture healing with $\mathrm{HRpQCT}$. Furthermore we advocate wearing the cast during all follow-up measurements to maintain the same scanning conditions.

\section{Appendix}

\section{Explanation for the different magnitude of errors in bone parameters introduced by the fiberglass and plaster-of-Paris cast}

The difference between the effect caused by a fiberglass cast and a plaster-ofParis cast can be explained by the different material properties of the two casts. At the standard settings of the XtremeCT, the mean energy of the photons is 40 $\mathrm{keV}$. At this energy, calcium sulfate, the main component of plaster-of-Paris cast, has a linear Xray attenuation coefficient $\mu$ of $2.07 \mathrm{~cm}^{-1}$. The main component of a fiberglass cast is silica, which has a $\mu$ of $1.23 \mathrm{~cm}^{-1}$ at $40 \mathrm{keV} .{ }^{20}$ Due to its higher $\mu$, the plaster-of-Paris cast absorbs more lower-energy photons than fiberglass, resulting in an underestimation of the bone density. 


\section{References}

1. Johnell, O. \& Kanis, J. Epidemiology of osteoporotic fractures. Osteoporos Int 16 Suppl 2, S3-7 (2005).

2. Rüedi, T.P. \& Murphy, W.M. AO principles of fracture management (Thieme, Stuttgart, Germany, 2000).

3. Hao, Y.J. et al. Changes of microstructure and mineralized tissue in the middle and late phase of osteoporotic fracture healing in rats. Bone 41, 631-8 (2007).

4. Wolfl, C. et al. Characteristics of bone turnover in the long bone metaphysis fractured patients with normal or low Bone Mineral Density (BMD). PLoS One 9, e96058 (2014).

5. Goldhahn, J. et al. Implications for fracture healing of current and new osteoporosis treatments: an ESCEO consensus paper. Calcif Tissue Int 90, 343-53 (2012).

6. de Jong, J.J. et al. Assessment of the healing process in distal radius fractures by highresolution peripheral quantitative computed tomography. Bone 64C, 65-74 (2014).

7. Meyer, U. et al. Early Changes in Bone Density, Micro-Architecture, Bone Resorption and Inflammation Predict the Clinical Outcome 12 Weeks After Conservatively Treated Distal Radius Fractures: An Exploratory Study. J Bone Miner Res (2014).

8. MacNeil, J.A. \& Boyd, S.K. Improved reproducibility of high-resolution peripheral quantitative computed tomography for measurement of bone quality. Med Eng Phys 30, 792-9 (2008).

9. Boutroy, S., Bouxsein, M.L., Munoz, F. \& Delmas, P.D. In vivo assessment of trabecular bone micro-architecture by high-resolution peripheral quantitative computed tomography. J Clin Endocrinol Metab 90, 6508-15 (2005).

10. Liu, X.S. et al. High-resolution peripheral quantitative computed tomography can assess microstructural and mechanical properties of human distal tibial bone. J Bone Miner Res 25, 746-56 (2010).

11. Engelke, K. et al. Short-term in vivo precision of BMD and parameters of trabecular architecture at the distal forearm and tibia. Osteoporos Int 23, 2151-8 (2012).

12. Wytch, R., Ashcroft, G.P., McKenzie, G. Wardlaw, D., Ledingham, W.M. Radiographic assessment of splinting bandages. Injury 22, 41-4 (1991).
13. Pialat, J.B., Burghardt, A.J., Sode, M., Link T.M. \& Majumdar, S. Visual grading of motion induced image degradation in high-resolution peripheral computed tomography: impact of image quality on measures of bone density and micro-architecture. Bone 50, 111-8 (2012).

14. Laib, A., Hauselmann, H.J. \& Ruegsegger, P. In vivo high-resolution 3D-QCT of the human forearm. Technology and health care: official journal of the European Society for Engineering and Medicine 6, 329-37 (1998).

15. Laib, A. \& Rüegsegger, P. Comparison of structure extraction methods for in vivo trabecular bone measurements. Computerized Medical Imaging and Graphics 23, 69-74 (1999).

16. Hilderbrand, T., Laib, A., Müller, R., Dequeker, J. \& Rüegsegger, P. Direct Three-Dimensional Morhpometric Analysis of Human Cancellous Bone: Microstructural Data from Spine, Femur, Iliac Crest, and Calcaneus. Journal of Bone an Mineral Research 17, 1167 1174 (1999).

17. Pistoia, W. et al. Estimation of distal radius failure load with micro-finite element analysis models based on three-dimensional peripheral quantitative computed tomography images. Bone 30, 842-8 (2002).

18. Dalzell, N. et al. Bone micro-architecture and determinants of strength in the radius and tibia: age-related changes in a populationbased study of normal adults measured with high-resolution pQCT. Osteoporos Int $\mathbf{2 0}$ 1683-94 (2009).

19. Gluer, C.C. et al. Accurate assessment of precision errors: how to measure the reproducibility of bone densitometry techniques. Osteoporos Int 5, 262-70 (1995).

20. Hubbell, J.H. \& Seltzer, S.M. (National Institute of Standards and Technology, Gaithersburg, MD., 2004).

21. Bonnick, S.L. Bone densitometry in clinical practice : application and interpretation (Humana Press, Totowa, N.J., 2004).

22. Paggiosi, M.A., Eastell, R. \& Walsh, J.S. Precision of high-resolution peripheral quantitative computed tomography measurement variables: influence of gender, examination site, and age. Calcif Tissue Int 94, 191-201 (2014). 



$$
\frac{b}{x}
$$




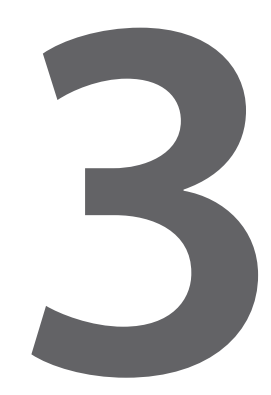

Assessment of the healing process in distal radius fractures by high-resolution peripheral quantitative computed tomography 


\section{Abstract}

\section{Background}

In clinical practice, fracture healing is evaluated by clinical judgment in combination with conventional radiography. Due to limited resolution, radiographs don't provide detailed information regarding the bone micro-architecture and bone strength. Recently, assessment of in vivo bone density, architectural and mechanical properties at the micro-scale became possible using high-resolution peripheral quantitative computed tomography (HRpQCT) in combination with micro finite element analysis ( $\mu \mathrm{FEA})$. So far, such techniques have been used mainly to study intact bone. The aim of this study was to explore whether these techniques can also be used to assess changes in bone density, micro-architecture and bone stiffness during fracture healing.

\section{Methods}

Therefore, the fracture region in eighteen women, aged 50 years or older with a stable distal radius fracture, was scanned using HRpQCT at one to two (baseline), three to four, six to eight and twelve weeks post-fracture. At one to two and twelve weeks postfracture the distal radius at the contra-lateral side was also scanned as control. Standard bone density, micro-architectural and geometric parameters were calculated and bone stiffness in compression, torsion and bending was assessed using $\mu$ FEA. A linear mixedeffect model with time post-fracture as fixed effect was used to detect significant ( $p$-value $\leq 0.05$ ) changes from baseline. Wrist pain and function was scored using the patient-rated wrist evaluation (PRWE) questionnaire. Correlations between the bone parameters and the PRWE score was calculated by Spearman's correlation coefficient.

\section{Results}

At the fracture site, total and trabecular bone density increased by $11 \%$ and $20 \%$, respectively, at six to eight weeks, whereas cortical density was decreased by $4 \%$. Trabecular thickness increased by $23 \%$ and $31 \%$ at six to eight and twelve weeks and the intertrabecular area became blurred, indicating intertrabecular bone formation. Compared to baseline, calculated bone stiffness in compression, torsion and bending were increased by $31 \%$ after twelve weeks. A moderate negative correlation was found between the stiffness and the PRWE score. No changes were observed at the contralateral side.

\section{Conclusion}

The results demonstrate that it is feasible to assess clinically relevant and significant longitudinal changes in bone density, micro-architecture and mechanical properties at the fracture region during the healing process of stable distal radius fractures using HRpQCT. 


\section{Introduction}

Fragility fractures of the distal radius are one of the most common fractures. The incidence is about $17 \%$ of all fractures. ${ }^{1}$ Although not always acknowledged, the outcome of distal radius fractures is not uniformly good. Poor outcomes have been attributed to poor restoration of anatomy and secondary loss of reduction after an initially adequate reduction ${ }^{2}$, leading to discomfort, loss of range of motion and/or soft tissue complaints. ${ }^{3-5}$

It is expected that the addition of medication or supplements, such as vitamin D or calcium can improve the clinical outcome of distal radius fractures. ${ }^{6}$ To monitor the process of fracture healing in patients, who either received supplemental medication or not, a method that is able to evaluate bone healing in detail is necessary. In clinical practice, fracture healing is evaluated by clinical judgment of the physician in combination with plain anteroposterior (AP) and lateral radiographs. However, these evaluations do not provide detailed information with regard to the healing process and consolidation of fractures on the level of cortical and trabecular bone micro-architecture.

Until recently, human bone micro-architecture could only be studied with bone biopsies, i.e. with 2D histomorphometry or 3D microCT, since imaging techniques that can provide sufficient resolution for bone structure in vivo were not available. Therefore, in vivo high-resolution imaging studies on fracture healing were restricted to animals. ${ }^{7,8}$ Classical QCT proved to be a successful estimator for the prediction of the mechanical stability of long bones ${ }^{9}$, but was not sufficient to study fracture healing in the distal radius in patients because of insufficient resolution to visualize individual trabeculae. ${ }^{10}$

With the development of a new low-dose radiation high-resolution peripheral quantitative computed tomography (HRpQCT) technique, i.e. XtremeCT, it is possible to assess in vivo bone density and architecture at the microscale. ${ }^{11-13}$ Moreover, biomechanical properties of the bone can be calculated by micro finite element analysis ( $\mu$ FEA) based on such HRpQCT images. ${ }^{11,14,15}$

So far, these high-resolution imaging techniques were mainly used to study micro-architectural changes due to ageing ${ }^{16,17}$, osteoporosis and other (bone) diseases $^{15,18,19}$ and the effect of different treatments ${ }^{20-23}$. These techniques, however, could be applied as well to analyze the process of bone healing after a distal radius fracture. Potentially, such analyses could provide a new tool to assess fracture consolidation based on bone morphology or $\mu$ FEA derived mechanical parameters. In a recent study, Mueller et al. ${ }^{24}$ investigated the feasibility of using these techniques to assess fracture healing in vivo and found an $18 \%$ increase in calculated stiffness over a period of two months. However, in that study biomaterials 
and plates were used to stabilize the fracture. The application of these techniques to fracture healing without any intervention, other than a standard cast, however, has not been demonstrated and it remains unclear to what extent the healing process can be captured by the standard morphological and mechanical parameters.

The objective of this study was, therefore, to explore the feasibility of HRpQCT in combination with $\mu F E A$ to assess longitudinal changes in bone density, microarchitecture and biomechanical parameters during the first twelve weeks of healing of conservatively treated distal radius fractures.

\section{Materials and Methods}

\section{Subjects}

Twenty women aged 50 years or older with a stable distal radius fracture, which was immobilized by a cast, were included in this study. All patients were included at the departments of Orthopedics and Traumatology of the Maastricht University Medical Center, the Netherlands. The exclusion criteria were: a history of previous fractures at the fractured side; known systemic or metabolic disorders leading to progressive bone deterioration; use of glucocorticoids; presence of an active inflammatory disease; presence of an active or suspected infection; or malignancy in the last twelve months pre-fracture.

To assess changes in bone density, micro-architecture, geometry and biomechanics, all patients underwent HRpQCT scanning of the fractured distal radius during four visits. The visits were scheduled one to two weeks post-fracture (baseline) and three to four weeks, six to eight weeks and twelve weeks postfracture. During the first and last visit, the radius at the contra-lateral side was scanned as well. The protocol (registration number NTR3821) was approved by an independent Medical Ethics Committee, and all patients gave written informed consent prior to participation.

\section{Scanning by HRPQCT}

During each visit, the fractured radius was imaged by HRpQCT (XtremeCT, Scanco Medical AG, Switzerland) using clinical in vivo settings by the manufacturer (effective energy of $60 \mathrm{kVp}$, tube current of $900 \mu \mathrm{A}$ and $100 \mathrm{~ms}$ integration time). The region of interest (Figure 1) was based on AP and lateral radiographs of the fractured radius, in which the proximal edge of the lunate was used as reference. To make sure the complete fracture was scanned, the scan length was set to $18 \mathrm{~mm}$. With an isotropic voxelsize of $82 \mathrm{~mm}$, each HRpQCT measurement thus resulted in 220 parallel CT slices. The effective dose of each HRpQCT measurement at the fracture side was 
less than $6 \mathrm{mSv}$. Because the patient's forearm was fixed in a cast, the forearm with the cast was placed in a custom cylindrical carbon holder with an inflatable cushion (Pearltec AG, Schlieren, Switzerland) to minimize patient motion. To scan the radius at the contra-lateral side, standard clinical in vivo settings by the manufacturer were used and resulted in one stack containing 110 parallel CT slices with an isotropic voxelsize of $82 \mathrm{~mm}$. The offset at the contra-lateral side was fixed for all patients and was chosen $9.5 \mathrm{~mm}$ from the proximal edge of the lunate. The volume between 9.5 and $18.5 \mathrm{~mm}$ from the lunate was thus scanned at the contra-lateral side. The same holder as for the fractured forearm was used to fixate the contra-lateral side.

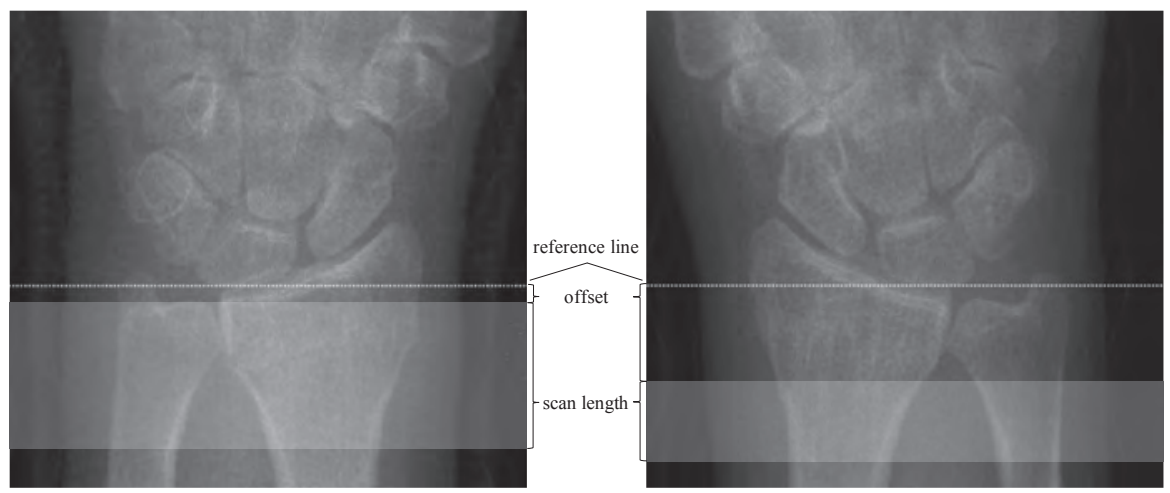

Figure 1 Region of interest at the fracture side (left) and the contra-lateral side (right). The scan length at the fracture side is set to $18 \mathrm{~mm}$ and contains 220 parallel HRpQCT slices, while the scan length at the contra-lateral side is set to $9 \mathrm{~mm}$ and contains 110 parallel HRpQCT slices. The offset at the fracture side varied between subjects, while the offset at the contra-lateral side was kept constant at a distance of 9.5 $\mathrm{mm}$ from the proximal edge of the lunate.

Each scan was checked for motion induced image artifacts and was quality graded by the operator according to the manufacturer's guidelines and as described by Pialat et al. ${ }^{25}$ In case such artifacts occurred and thus resulted in images of insufficient quality, i.e. grade 4 or 5 , the scan was repeated.

\section{Evaluation of bone density, micro-architecture and geometry}

The HRpQCT images were evaluated using the standard patient evaluation protocol provided by the manufacturer, which has been described earlier in detail. ${ }^{26}$ In short, the cortical and trabecular region were separated first using a semi-automated contouring scheme in which the periosteal boundary surface of the radius was derived. ${ }^{27}$ After contouring, a Laplace-Hamming filter (epsilon 0.5 and cut-off frequency 0.4 ) followed by normalization (range 0-1000) and global thresholding (threshold 400), was used in order to extract the voxels that represented mineralized bone and to create the segmented images. The used values were default values 
specified by the manufacturer and these are consistently used for all HRpQCT scanners.

The following bone density parameters were calculated from the images: volumetric bone mineral density $[\mathrm{mgHA} / \mathrm{cm} 3]$ was assessed for the total region (Dtot) and the trabecular (Dtrab) and cortical region (Dcort) separately.

The micro-architectural parameters that were used in this study were: the trabecular number (Tb.N) [1/mm], thickness (Tb.Th) $[\mathrm{mm}]$ and separation (Tb.Sp) [mm], which are all determined using a 3D ridge extraction method ${ }^{28}$ and standard morphological relations. Bone geometry was expressed by the cortical thickness (Ct.Th) [mm], which is calculated by dividing the cortical volume by the outer cortical surface, and the cortical perimeter (Ct.Pm) [mm], which is the average outer perimeter of the cortex.

\section{uFE analysis}

With the standard approach for $\mu \mathrm{FE}$ analyses as used in most earlier studies, segmented images are used to create a representative $\mu \mathrm{FE}$ model of the bone's micro-architecture by converting each voxel that represents bone tissue into a brick element of the same size. Material properties then are chosen constant for all bone tissue ${ }^{16}$, or varied only between cortex and trabecular bone tissue. ${ }^{15}$ Whereas this approach has been well validated and successfully applied in clinical studies, its application to fractured bone is not trivial. The use of segmented images clearly limits the models to only represent the mineralized phase. In particular in the early stages of fracture healing this approach is expected to be inaccurate because it does not account for newly formed low mineralized bone. In an earlier study, Shefelbine et al. therefore introduced a more sophisticated approach that can account for the stiffness of less mineralized tissues as well. ${ }^{8}$ With their approach, three different tissue types are distinguished (soft tissue, low mineralized tissue and high mineralized tissue) and the stiffness of the elements representing the mineralized tissue was made dependent on its material composition.

In the present study, we applied both the standard approach, which is validated for intact bone, and the approach proposed by Shefelbine et al., which is validated for healing bone. Since the method of Shefelbine et al. was originally developed for micro-CT data with a higher resolution, it was slightly adapted to apply it to the HRpQCT data used in this study. In short, the grayscale bone density images were thresholded to define three separate regions: soft tissue, newly mineralized bone that had less mineral content, and highly mineralized bone using thresholds of 0-4000, 4001-5750 and 5751-max. (2-byte signed short-integer values, equal to $<343,343-642$ and $>642 \mathrm{mgHA} / \mathrm{cm}^{3}$ ), respectively. Elastic moduli were assigned to each region in agreement with Shefelbine et al.: $50 \mathrm{MPa}$ for soft-tissue, 5000 
MPa for the newly mineralized bone, and 15000 MPa for highly mineralized bone. Following, the model was downscaled by a factor two and each downscaled voxel was converted into a brick element, such that the final element size was $164 \mathrm{~mm}$, which is similar to the element size used in the study by Shefelbine et al. (200 mm). In the resulting $\mu \mathrm{FE}$ model soft tissue was assigned a constant modulus of $50 \mathrm{MPa}$ whereas for mineralized bone a Young's modulus ranging from 5000 to $15000 \mathrm{MPa}$ results, depending on the material composition of the downscaled voxel (Figure 2). A Poisson's ratio of 0.3 was assigned to all elements and all materials were assumed to be linear elastic and isotropic.

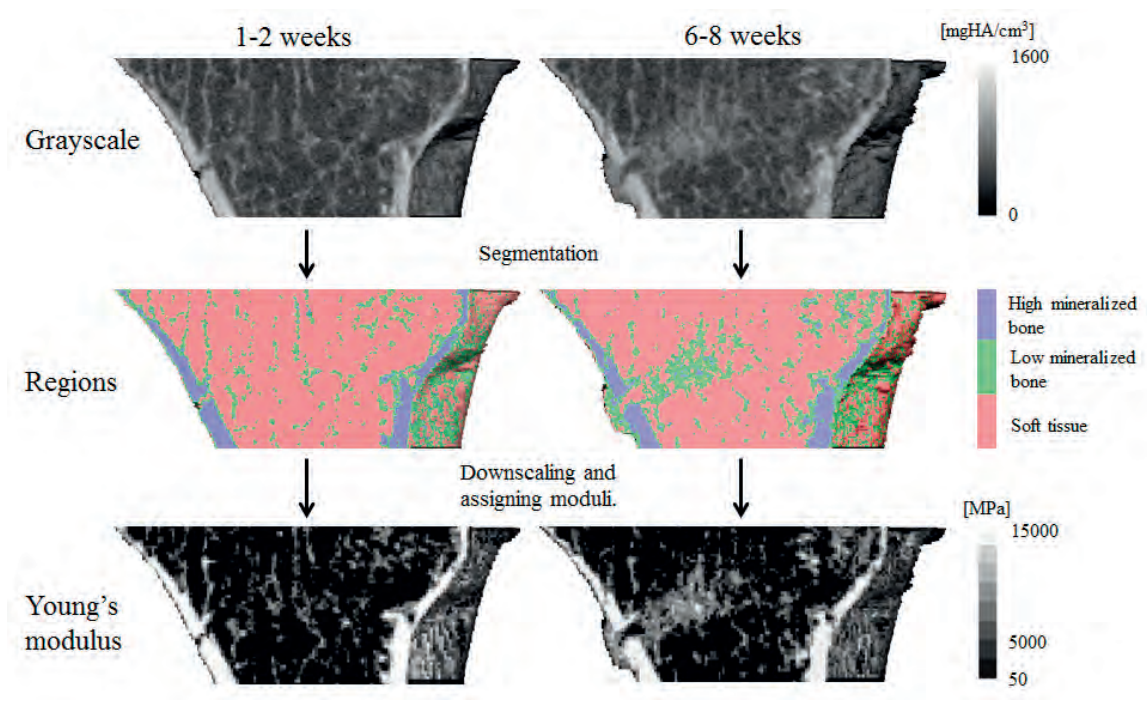

Figure 2 Creation of the voxel finite element models fromHRpQCT scans based on Shefelbine's method. From the grayscale images, three regions representing soft tissue and low and high mineralized bone were segmented. The elastic modulus was linearly scaled to the gray value for the bone regions resulting in moduli ranging from 5000 to $15000 \mathrm{MPa}$. A modulus of $50 \mathrm{MPa}$ was assigned to all soft tissue. (Left: One to two weeks post-fracture, right: six to eight weeks post-fracture).

With the standard $\mu \mathrm{FE}$ approach, a procedure similar to that described in earlier studies was used and all elements were assigned a Young's modulus of $10 \mathrm{GPa}$ and a Poissons ratio of $0.3 .16,29,30$

By subsequently applying different load cases, clinically relevant loading scenarios were simulated and the associated stiffness was chosen as outcome variable. The first load case represented a 'high friction' compression test with a prescribed displacement in the axial direction of $1 \%$ of the total length, from which the compression stiffness (Scomp) [kN/mm] was calculated. The second load case represented a prescribed rotation of $0.01 \mathrm{rad}$ around the longitudinal axis applied to the surface perpendicular to this axis from which the torsional stiffness (Stors) 
[kNmm/rad] was calculated. A third and fourth load case represented a prescribed rotation of $0.01 \mathrm{rad}$ applied around the sagittal and transversal axes respectively, onto the surfaces perpendicular to each axis, thus inducing a state of pure bending in two directions, from which the bending stiffness in each direction (S.sagittal and S.transversal) was calculated. Because the orientation of the patients' forearm was not exactly the same during all visits, a quadratic mean bending stiffness was calculated (Sbend) $\left[\mathrm{kNmm}^{2} / \mathrm{rad}\right]$ from the sagittal and transversal bending stiffness according to Equation 1.

$$
\text { Sbend }=\sqrt{\frac{\text { Ssagittal }^{2}+\text { Stransversal }^{2}}{2}}
$$

\section{Clinical and radiological outcome}

Pain and function were measured using a validated Dutch version of the PatientRated Wrist Evaluation (PRWE) questionnaire. The PRWE questionnaire consists of ten questions and its score ranges from 0 (no pain/no difficulty) to 100 (worst pain/disability). It has previously been shown that PRWE is a reliable and valid measurement tool after distal radius fractures. ${ }^{31,32}$

Based on AP and lateral radiographic images of the fractures, the state of consolidation was assessed at each visit by an experienced orthopedic surgeon. The state of consolidation was defined as none when there was no sign of cortical bridging or increased radio density along the fracture line; as incomplete when there was increased radio density along the fracture line and starting cortical bridging was observed; and as complete when fading of the initial fracture line was seen in combination with cortical bridging. ${ }^{33}$

\section{Statistics}

The Shapiro-Wilk test was used to test if the bone parameters were normally distributed at all visits. Non-normally distributed parameters were log-transformed. Then, a linear mixed effect model with the time post-fracture as fixed effect was used to test if the bone parameters at the follow-up visits were significantly different from baseline. The bone density, architectural and mechanical parameters were expressed as estimated marginal means with 95\% confidence intervals at each visit. Percent change from baseline in each parameter was calculated by dividing the difference from baseline by the mean value at baseline. Spearman's correlation coefficient was used to measure the correlation between the bone parameters and the clinical and radiological outcome. Statistical analyses were performed with SPSS Statistics for Windows version 20.0 (IBM Corp., Armonk, USA). 


\section{Results}

From the twenty patients who were initially included in the study, one patient declined participation at the first visit and another patient at the second visit. Their results were not included in this study. The characteristics of the remaining eighteen patients are presented in Table 1.

Table 1 Descriptive baseline characteristics of the eighteen women that completed all study visits

\begin{tabular}{lc}
\hline & $\begin{array}{c}\text { Mean } \pm \text { Std. Dev. } \\
(\mathrm{n}=18)\end{array}$ \\
\hline Age [years] & $64 \pm 8$ \\
Weight [kg] & $70 \pm 8$ \\
BMI [kg/m2] & $27 \pm 4$ \\
Time since menopause [years] & $13 \pm 9$ \\
T-score total hip [-] & $-1.4 \pm 1.1$ \\
T-score lumbar spine [-] & $-2.4 \pm 1.5$ \\
25(OH)D level [nmol/L] & $63 \pm 29$ \\
25(OH)D level <50 nmol/L [n] & $7(39 \%)$ \\
\hline
\end{tabular}

$\mathrm{BMI}=$ body mass index; $25(\mathrm{OH}) \mathrm{D}=25$-hydroxyvitamin $\mathrm{D}$

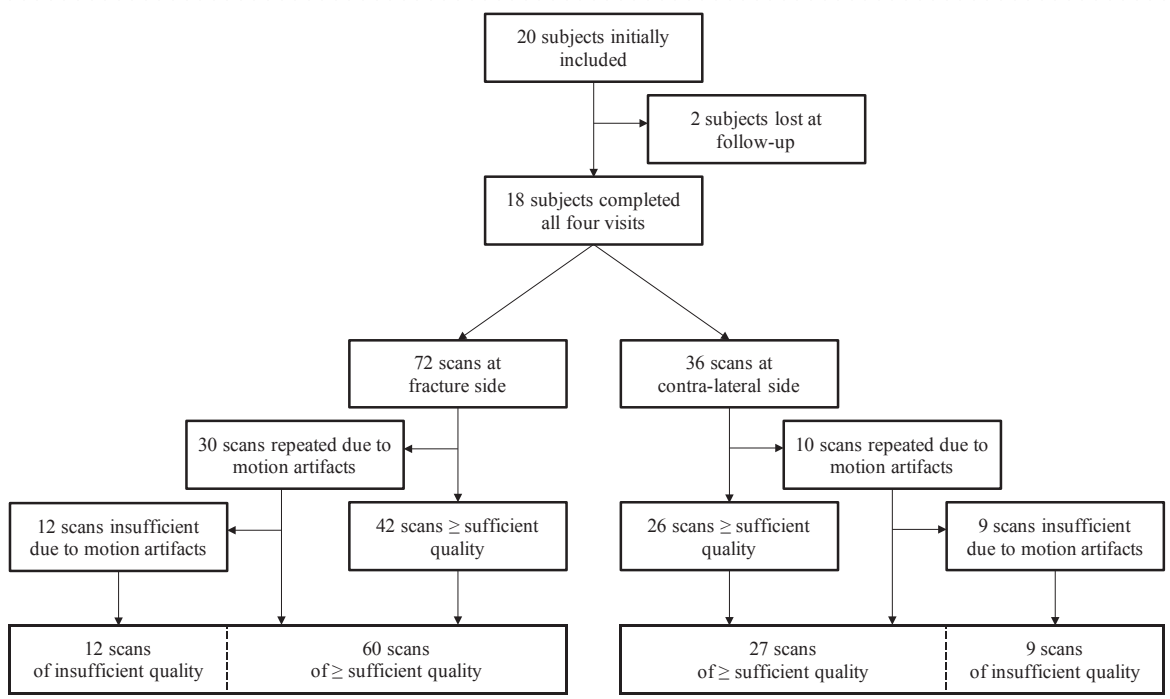

Figure 3 Overview of HRpQCT scans that were included in the evaluation of the bone density, microarchitectural, geometrical and biomechanical parameters. With a maximum of one repeated measurement in case motion artifacts occurred, $83 \%$ of the scans at the fracture side and $75 \%$ of the scans at the contra-lateral side were finally of sufficient quality.

From the scans in the eighteen patients, 40 out of a total of 108 scans (37\%) were initially of insufficient quality due to motion artifacts: 30 out of 72 scans at the 
fracture side and 10 out of 36 at the contra-lateral side. After repeating these scans, still $19 \%$ of the scans were of insufficient quality due to motion-induced image artifacts. These scans were not included in the analyses, thus in total 87 scans were used for the evaluation of the bone parameters (Figure 3).

\section{Visual assessment}

The sequential HRpQCT images showed a common pattern at the fracture region over time (Figure 4). In general, the fracture line was clearly visible at one to two weeks post-fracture: the cortex was disrupted on several locations and the trabeculae along the fracture line were disrupted. At three to four weeks post-fracture, the trabecular region became blurred, i.e. the individual trabeculae were hard to distinguish. This blurring in the trabecular region was more

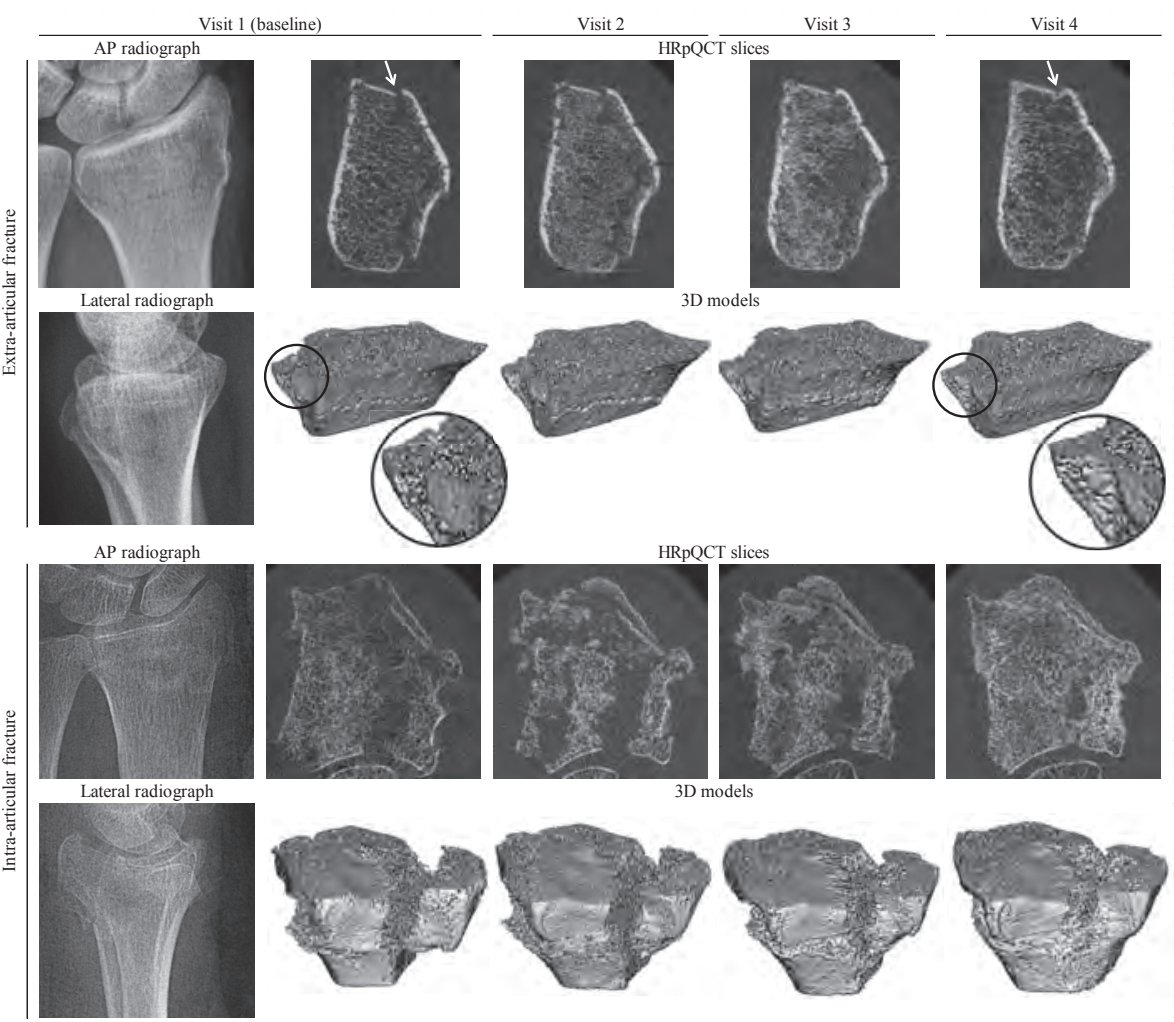

Figure 4 Anteroposterior (AP) and lateral radiographs at baseline and HRpQCT slices and 3D models at baseline and follow-up visits of an extra-articular (top) and an intra-articular (bottom) fracture that were included in this study. Compared to the radiographs, the initial fracture is much more detailed visible in the HRpQCT slices and the 3Dmodels. Furthermore, the increase in density in the trabecular region as well as the bridging of the cortex (arrows) is very well visualized in the HRpQCT slices and 3D models (zoom-in). 


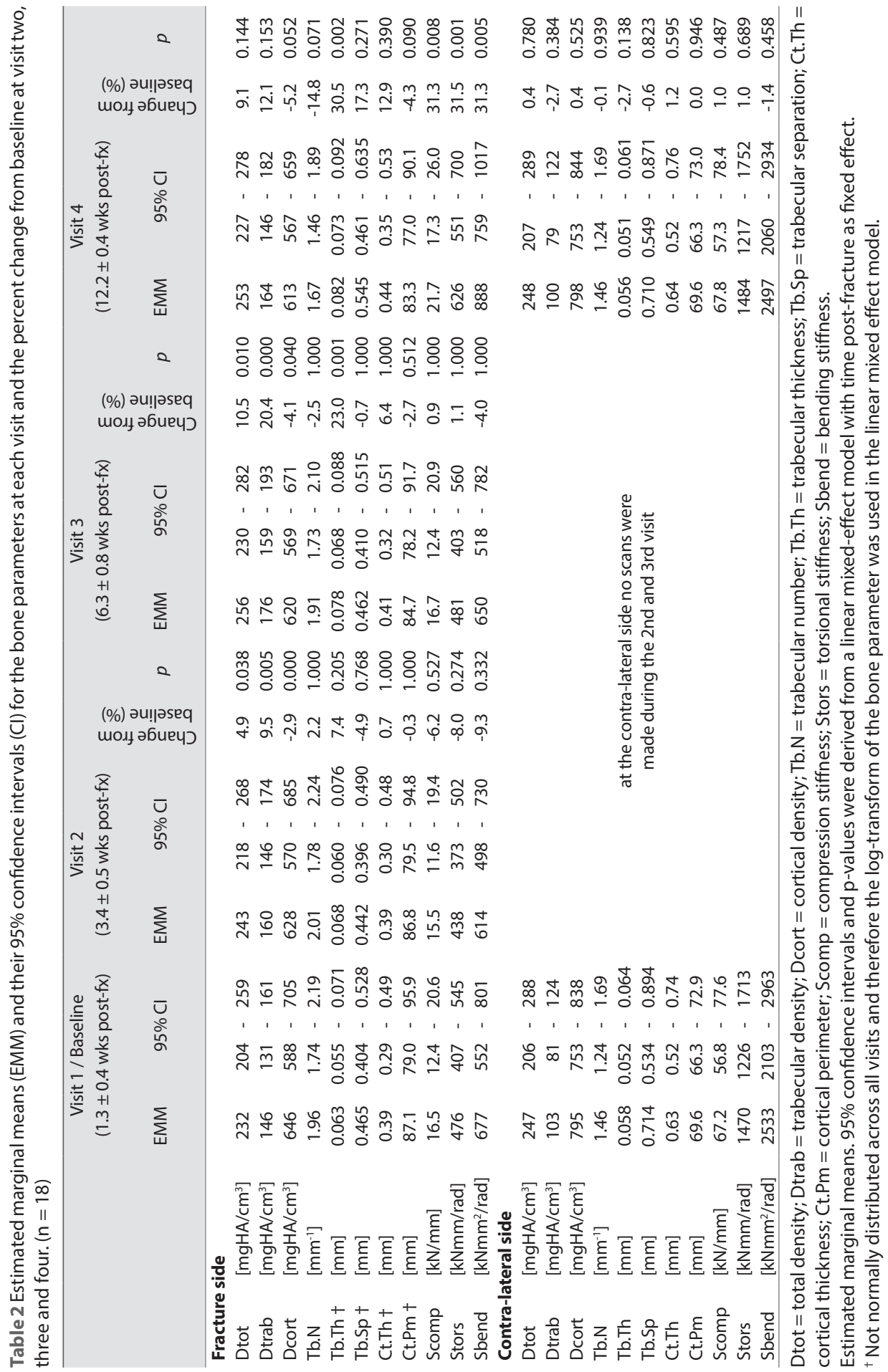


pronounced at six to eight weeks post-fracture and decreased at twelve weeks post-fracture. Bridging of the cortex started at six to eight weeks post-fracture and was completed at twelve weeks post-fracture. No changes were observed at the contra-lateral side.

\section{Quantitative assessment}

Estimated marginal means, 95\% confidence intervals and percent change from baseline for the bone parameters measured at the fractured side as well as the contra-lateral side are given in Table 2. Time series line-plots of the average percent change from baseline in the bone parameters are shown in Figure 5.

At the fracture side, total bone density increased by $10.5 \%(p>0.01)$ at six to eight weeks post-fracture and showed a final change of $+9.1 \%(p>0.05)$ at twelve weeks post-fracture (Figure 5A). This was the result of cumulative, opposite changes in trabecular and cortical bone density. Whereas trabecular bone density (Figure 5B) significantly increased to a peak of $20.4 \%(p<0.01)$ at six to eight weeks post-fracture and a final increase of $12.1 \%(p>0.05)$ at twelve weeks post-fracture, cortical density decreased progressively to $-5.2 \%(p>0.05)$ at twelve weeks postfracture (Figure 5C).

Trabecular thickness significantly increased by $23.0 \%(p<0.01)$ after six to eight weeks up to $30.5 \%$ ( $p<0.01$ ) at twelve weeks post-fracture (Figure $5 \mathrm{E}$ ). Trabecular number remained constant until three to four weeks post-fracture and subsequently tended to decrease by $14.8 \%$ ( $p>0.05$ ) at twelve weeks post-fracture (Figure 5D). No significant changes were observed in trabecular separation (Figure $5 F)$.

There were no significant changes in cortical thickness (Figure 5G) and cortical perimeter during the twelve weeks post-fracture (Figure $5 \mathrm{H}$ ).

Bone stiffness in compression, torsion and bending calculated by the approach of Shefelbine et al. (Figure $5 \mathrm{I}, 5 \mathrm{~J}$ and $5 \mathrm{H}$, respectively) showed no significant change until twelve weeks post-fracture. At that time-point they were increased by $31.3 \%$, $31.5 \%$ and $31.3 \%$ (all $p<0.01$ ), respectively. Bone stiffness in compression, torsion and bending calculated by the standard approach (data not shown in Table) showed a similar behavior, but were increased by $38.4 \%, 46.8 \%$ and $43.0 \%$ (all $p<$ $0.02)$, respectively.

At the contra-lateral side, no significant changes from baseline were observed in any of the bone parameters.

\section{Correlation with clinical and radiological outcome}

PRWE score correlated significantly with trabecular thickness ( $r$ o $=-0.43, p=$ 0.001 ) and stiffness in compression ( $r$ o $=-0.28, p=0.029$ ), torsion (rho $=-0.29$, 

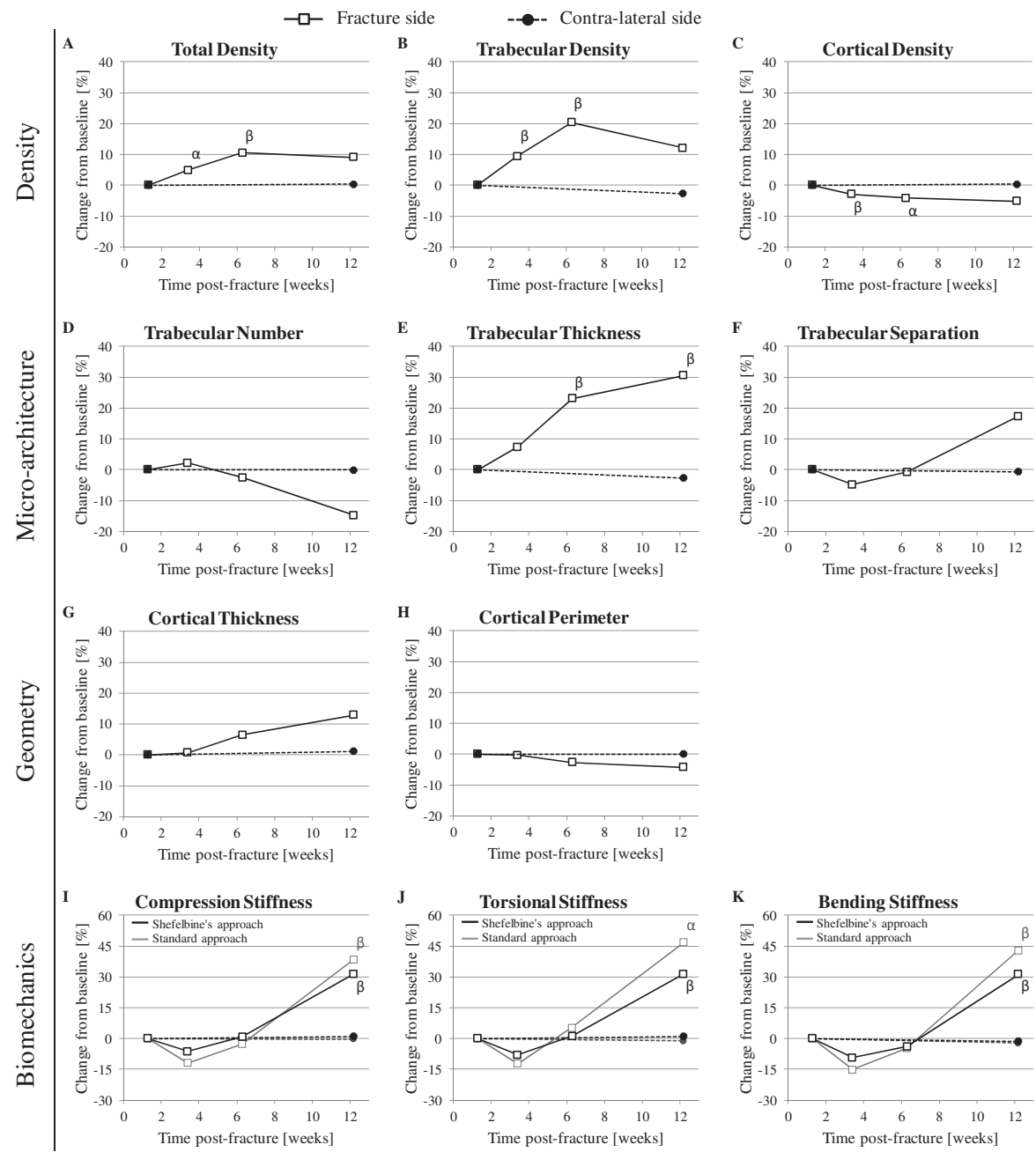

Figure 5 Time series line-plots of the percent change from baseline in HRpQCT derived bone density, micro-architectural, geometrical and biomechanical parameters at the fracture side (white squares) and the contra-lateral side (black circles). a significantly different from baseline, $p<0.05$. $\beta$ significantly different from baseline, $\mathrm{p}<0.01$.

$p=0.024)$ and bending ( $r h o=-0.30, p=0.019$ ) as calculated by Shefelbine's approach (Table 3). Similar correlations were found for the stiffnesses calculated with the standard $\mu \mathrm{FEA}$ approach: compression stiffness ( $r$ o $=-0.33, p=0.011$ ), torsional stiffness ( $r h o=-0.32, p=0.012$ ), and bending stiffness ( $r$ o $=-0.37, p=$ 0.004) (Table 3).

The radiological state of consolidation correlated significantly with trabecular thickness ( $r$ o $=0.35, p=0.006$ ), cortical perimeter (rho $=-0.41, p=0.001$ ) and stiffness in compression ( $r$ ho $=0.44, p<0.001$ ), torsion ( $r$ ho $=0.35, p=0.006$ ) 
and bending ( $r$ o $=0.37, \mathrm{p}=0.004$ ) as calculated by Shefelbine's approach (Table 3). Using the standard $\mu$ FEA approach, similar correlations were obtained: compression stiffness ( $r$ ho $=0.50, p<0.001$ ), torsional stiffness ( $r$ o $=0.44, p<$ 0.001), and bending stiffness ( $r$ o $=0.41, p=0.001$ ) (Table 3 ).

No correlations were found between the other bone parameters and the PRWE score or the radiological state of consolidation.

Because the stiffness at the first visit is probably overestimated, we also calculated the correlation not taking the first visit into account. Then, the correlations between the PRWE score and the stiffness in compression, torsion and bending as calculated by Shefelbine's approach increase to $-0.37(p=0.012),-0.40$ $(p=0.006)$ and $-0.40(p=0.007)$, respectively. And the correlations between the PRWE score and the stiffnessess calculated by standard $\mu$ FEA approach increase to $-0.44(p=0.002),-0.35(p=0.017)$ and $-0.38(p=0.009)$ for the stiffness in in compression, torsion and bending, respectively.

Table 3 Correlations between the bone stiffness parameters and the PRWE score as well as the radiological state of consolidation.

\begin{tabular}{lcccc}
\hline Bone stiffness & \multicolumn{2}{c}{ PRWE score } & \multicolumn{2}{c}{$\begin{array}{c}\text { Radiological state of } \\
\text { consolidation }\end{array}$} \\
\hline Scomp (Shefelbine FEA) & rho & p-value & rho & p-value \\
Stors (Shefelbine FEA) & -0.282 & 0.029 & 0.437 & $<0.001$ \\
Sbend (Shefelbine FEA) & -0.291 & 0.024 & 0.353 & 0.006 \\
Scomp (standard FEA) & -0.302 & 0.019 & 0.365 & 0.004 \\
Stors (standard FEA) & -0.327 & 0.011 & 0.500 & $<0.001$ \\
Sbend (standard FEA) & -0.322 & 0.012 & 0.441 & $<0.001$ \\
\hline
\end{tabular}

Scomp = compression stiffness; Stors = torsional stiffness; Sbend = bending stiffness; FEA = finite element analysis. Correlations were calculated using Spearman's correlation coefficient (rho).

\section{Discussion}

The main objective of this study was to explore the feasibility of HRpQCT based techniques to study the fracture healing process of conservatively treated distal radius fractures in vivo. The results show that it is possible to detect significant changes in HRpQCT derived bone densitometric, micro-architectural and mechanical parameters during the first twelve weeks of fracture healing using HRpQCT in combination with $\mu$ FEA. These results were obtained by performing the standard patient evaluation protocols that have been provided by the manufacturer and are readily available on every XtremeCT device. Although the contouring of the periosteal boundary often required manual correction due to the disruptions in the cortical surface, the calculation of the bone density and micro-architectural 
parameters could proceed in the standard way. Also the segmented 3D models could be used in the standard $\mu$ FEA without problems.

The time-related increase in trabecular bone density with a peak at six to eight weeks post-fracture was the most striking finding. At the time point of this peak increase, trabecular density was significantly increased and the trabecular structure, when visually assessed, became blurred (Figure 4). This finding might reflect the formation of new woven bone between the trabeculae during early stages of distal radius fracture healing, which is supported by the histological findings reported by Aspenberg et al. ${ }^{34}$ At twelve weeks post-fracture, when the change from baseline in trabecular density was not significantly increased anymore, trabecular thickness further increased and the largest increase in calculated stiffness was observed. We hypothesize that the formation of new woven bone in the trabecular region in the first weeks post-fracture is followed by extensive remodeling between week six and twelve, when poorly mineralized unorganized woven bone is removed and replaced by normal well-organized bone tissue, with improved bone strength but a decrease of trabecular density at twelve weeks compared to six weeks postfracture. ${ }^{35,36} \mathrm{~A}$ similar process, where the increase in bone density precedes the micro-architectural adaptation, has been described in developing bone. ${ }^{37}$

Although we do detect significant changes in structural parameters, the interpretation of these changes in the healing bone might not be the same as in intact bone and warrants additional discussion. The presence of woven bone at three to four and six to eight weeks post-fracture, i.e. when the images show a more blurred region, influences the calculation of several parameters that are used to quantify the trabecular structure. With the standard evaluation as used here, the trabecular thickness and separation are derived from trabecular bone density, via bone volume fraction, and trabecular number according to standard histomorphometric relations. ${ }^{26}$ Because of its finer structure and lower density not all woven bone is segmented after thresholding and, consequently, not included in the calculation of the trabecular number. It does, however, increase trabecular bone density. These combined effects lead to a significant increase in the calculated trabecular thickness parameter that, however, does not reflect an actual increase of the thickness of trabeculae. This shows that one must take care with the interpretation of the structural parameters as they could represent a different morphological aspect in fractured bone than in intact bone. A second point that should be mentioned here is that the morphological parameters represent the average values for the whole analyzed region. The measured changes in bone morphology parameters thus are dependent on the size of the analyzed region 
and that of the fracture: if the fracture comprises a small part of the analyzed volume, changes in morphology parameters will appear to be smaller than in the case where the fracture occupies larger regions. The $\mu$ FEA parameters, however, are less sensitive to the size of the analyzed region and that of the fracture: even a small fracture can dramatically reduce the stiffness of the bone.

Despite the fact that the images also showed large local changes in the cortical region during the healing process, i.e. bridging of the cortex, the changes measured in cortical thickness were not significant. The calculation of cortical thickness, however, is the average of the whole cortical region and appears to be insensitive to these local changes. This finding suggests that a more localized approach is needed to describe the healing process in the cortical compartment.

\section{Restoration of bone stiffness}

Restoration of bone strength and stiffness is of particular interest during the healing process of fractures. Although the approach by Shefelbine et al. that was used in the finite element analysis was only validated for femoral fractures in rats ${ }^{8}$, it is currently the only validated method reported in literature to assess the fracture stiffness in healing calluses through high-resolution CT, taking the properties of the callus into account. Regardless of this difference in fracture site and species and the fact that callus properties might vary when derived from CT data, the FEA results show that major changes from baseline are measured at twelve weeks postfracture. Since a similar development in stiffness was calculated with the standard $\mu$ FEA approach, it might not make a big difference which approach is used. However, particular during the early stages of fracture healing we propose to incorporate the callus into the model and therefore use the method of Shefelbine et al. At later stages, when the callus becomes less prominent, the standard approach might suffice since the results acquired by both approaches converge to each other: at twelve weeks post-fracture the correlation between the compression stiffnesses calculated by the two methods is strong with an $\mathrm{R}^{2}$ of 0.81 .

The finding that both approaches indicate that the stiffness first tends to decrease and later increases could be due to the fact that we compared changes to the baseline case. It is possible that the stiffness at baseline is overestimated by the FEA because the imaging procedure can identify touching trabeculae in the fractured region as connected. This might affect the standard $\mu$ FEA procedure more than Shefelbine's approach, hence this could explain why the final increase in compression stiffness calculated with the standard $\mu$ FEA approach (38.4\%) is more pronounced than with the Shefelbine approach (31.1\%).

Interestingly, the significant correlation between the PRWE score and the bone stiffness parameters suggests an association of this clinical parameter and 
HRpQCT derived stiffness. When the first visit is not taken into account because of the suspected overestimation at this time-point, the Spearman's correlation coefficients are even higher for all stiffnesses.

\section{Limitations}

This study has several limitations. First, as was mentioned before, the interpretation of structural parameters is not the same as that for intact bone, in particular when considering parameters such as trabecular thickness. Although we found significant changes in the parameters, one should be careful with the interpretation of such changes. It has not been proven in this study that these changes are a meaningful representation of the bone consolidation during healing. The same holds for the results obtained from the $\mu \mathrm{FE}$ analyses. Although the higher resolution makes it possible to better detect the fracture, we presently cannot prove that the predicted changes in stiffness are realistic, nor can we prove that the results of $\mu F E A$ are more accurate than those of continuum FEA based on clinical CT scans. A comparative study involving both continuum FE modeling and $\mu \mathrm{FE}$ modeling will be needed to further address these issues, but this was beyond the scope of the present paper.

Second, the region that was scanned at the contra-lateral side differed from the one at the fracture side. Thus we can only claim that nothing has changed at the contra-lateral side, but we cannot compare both sides to each other, which would have been very interesting. For example, it is unknown to what extent the stiffness at the fracture side has been restored to the pre-fracture stiffness. Since good correlations between the left and right radius has been reported ${ }^{38}$, the stiffness at the contra-lateral side might be regarded as the pre-fracture stiffness. To be able to compare both sides, we recommend matching the location of the scanned volume at the contra-lateral side to the one at the fracture side in future studies.

Despite the correlation between the PRWE score and the bone stiffness, a third limitation of this study is that the two $\mu$ FEA methods that were applied are not validated in human distal radius fractures. It will be hard or even impossible to address this limitation adequately, i.e. experimentally measure bone strength in healing fractures in humans and compare it to the bone strength calculated from the $\mu F E A$, since the validation would raise some serious ethical concerns. Alternatively, validation using an animal metaphyseal fracture healing model could be performed in the future.

Last, as can be seen in the 2D images and 3D models, we observed that the fracture healing process has not been fully completed at twelve weeks in most women. In future research, therefore, it is recommended to extend the follow-up period, e.g. to 24 weeks, to get a better end-point of the fracture healing process. 
In conclusion, this study shows that it is possible to detect significant changes in HRpQCT derived bone density, micro-architectural and mechanical parameters during the first twelve weeks of healing of stable distal radius fractures. These results open a window to further explore the possibilities of HRpQCT to monitor fracture healing in detail. It is recommended that future work should focus on the validation of the FEA approach and the development of new relevant parameters that benefit from the resolution of HRpQCT.

\section{Acknowledgements}

The authors would like to thank Liesbeth Jutten and Margareth Winants from the Trialbureau of the department of Orthopedics for their efforts during the study. 


\section{References}

1. Tarantino, U. et al. The incidence of hip, forearm, humeral, ankle, and vertebral fragility fractures in Italy: results from a 3-year multicenter study. Arthritis Res Ther 12, R226 (2010).

2. Jupiter, J., Ring, D. \& PP, W. Surgical treatment of redisplaced fractures of the distal radius in patients older than 60 years. J Hand Surg Am 27, 714-723 (2002).

3. Rogachefsky, R., Lipson, S., Applegate B, O., EA, Savenor, A. \& McAuliffe, J. Treatment of severly comminute intra-articular fractures of the distal end of the raius by open reduction and combined internal and external fixation. J Bone Joint Surg Am 83, 509-519 (2001).

4. Fernandez, D. \& Geissler, W. Treatment of displaced articular fractures of the radius. $J$ Hand Surg Am 16, 375-384 (1991).

5. Heim, D., Stricker, U. \& Rohrer, G. Carpal tunnel syndrome after trauma. Swiss Surg 8, 15-20 (2002).

6. Doetsch, A.M. et al. The effect of calcium and vitamin D3 supplementation on the healing of the proximal humerus fracture: a randomized placebo-controlled study. Calcif Tissue Int 75, 183-8 (2004).

7. Morgan, E.F. et al. Micro-computed tomography assessment of fracture healing: relationships among callus structure, composition, and mechanical function. Bone 44, 335-44 (2009).

8. Shefelbine, S.J. et al. Prediction of fracture callus mechanical properties using microCT images and voxel-based finite element analysis. Bone 36, 480-8 (2005).

9. Augat, P., Merk, J., Genant, H. \& Claes, L. Quantitative assessment of experimental fracture repair by peripheral computed tomography. Calcif Tissue Int. 60, 194-199 (1997).

10. Grigoryan, M. et al. Quantitative and qualitative assessment of closed fracture healing using computed tomography and conventional radiography. Acad Radiol. 10, 1267-1273 (2003).

11. Liu, X.S. et al. High-resolution peripheral quantitative computed tomography can assess microstructural and mechanical properties of human distal tibial bone. $J$ Bone Miner Res 25, 746-56 (2010).
12. Boutroy, S., Bouxsein, M.L., Munoz, F. \& Delmas, P.D. In vivo assessment of trabecular bone micro-architecture by high-resolution peripheral quantitative computed tomography. The Journal of clinical endocrinology and metabolism 90, 6508-15 (2005).

13. MacNeil, J.A. \& Boyd, S.K. Accuracy of high-resolution peripheral quantitative computed tomography for measurement of bone quality. Med Eng Phys 29, 1096-105 (2007).

14. Macneil, J.A. \& Boyd, S.K. Bone strength at the distal radius can be estimated from highresolution peripheral quantitative computed tomography and the finite element method. Bone 42, 1203-13 (2008).

15. Vilayphiou, N. et al. Finite element analysis performed on radius and tibia HRpQCT images and fragility fractures at all sites in postmenopausal women. Bone 46, 1030-7 (2010).

16. Dalzell, N. et al. Bone micro-architecture and determinants of strength in the radius and tibia: age-related changes in a populationbased study of normal adults measured with high-resolution pQCT. Osteoporos Int 20, 1683-94 (2009).

17. Khosla, S. et al. Effects of sex and age on bone microstructure at the ultradistal radius: a population-based noninvasive in vivo assessment. J Bone Miner Res 21, 124-31 (2006).

18. Bacchetta, J. et al. Early impairment of trabecular micro-architecture assessed with HRpQCT in patients with stage II-IV chronic kidney disease. J Bone Miner Res 25, 849-57 (2010).

19. Romme, E.A. et al. Bone stiffness and failure load are related with clinical parameters in men with chronic obstructive pulmonary disease. J Bone Miner Res 28, 2186-93 (2013).

20. Burghardt, A.J. et al. A longitudinal HRpQCT study of alendronate treatment in postmenopausal women with low bone density: Relations among density, cortical and trabecular micro-architecture, biomechanics, and bone turnover. J Bone Miner Res 25, 2558-71 (2010).

21. Chapurlat, R.D. et al. Effect of oral monthly ibandronate on bone micro-architecture in women with osteopenia-a randomized placebo-controlled trial. Osteoporos Int 24 311-20 (2013). 
22. Rizzoli, R. et al. Effects of strontium ranelate and alendronate on bone microstructure in women with osteoporosis. Results of a 2-year study. Osteoporos Int 23, 305-15 (2012).

23. Seeman, E. et al. Risedronate reduces microstructural deterioration of cortical bone accompanying menopause. Osteoporos Int 21 (2010).

24. Mueller, T.L. et al. Non-invasive bone competence analysis by high-resolution pQCT: an in vitro reproducibility study on structural and mechanical properties at the human radius. Bone 44, 364-71 (2009).

25. Pialat, J.B., Burghardt, A.J., Sode, M., Link, T.M. \& Majumdar, S. Visual grading of motion induced image degradation in high-resolution peripheral computed tomography: impact of image quality on measures of bone density and microarchitecture. Bone 50, 111-8 (2012).

26. Laib, A., Hauselmann, H.J. \& Ruegsegger, P. In vivo high-resolution $3 \mathrm{D}-\mathrm{QCT}$ of the human forearm. Technology and health care 6, 32937 (1998).

27. Laib, A. \& Rüegsegger, P. Comparison of structure extraction methods for in vivo trabecular bone measurements. Computerized Medical Imaging and Graphics 23, 69-74 (1999).

28. Hilderbrand, T., Laib, A., Müller, R., Dequeker, J. \& Rüegsegger, P. Direct Three-Dimensional Morhpometric Analysis of Human Cancellous Bone: Microstructural Data from Spine, Femur, Iliac Crest, and Calcaneus. Journal of Bone an Mineral Research 17, 1167 1174 (1999).

29. Pistoia, W. et al. Estimation of distal radius failure load with micro-finite element analysis models based on three-dimensional peripheral quantitative computed tomography images. Bone 30, 842-8 (2002).
30. Chevalley, T., Bonjour, J.P., van Rietbergen, B., Ferrari, S. \& Rizzoli, R. Fractures during childhood and adolescence in healthy boys: relation with bone mass, microstructure, and strength. J Clin Endocrinol Metab 96, 3134-42 (2011).

31. MacDermid, J.C. Development of a scale for patient rating of wrist pain and disability. $J$ Hand Ther 9, 178-83 (1996).

32. Brink, S.M., Voskamp, E.G., Houpt, P. \& Emmelot, C.H. Psychometric properties of the Patient Rated Wrist/Hand Evaluation Dutch Language Version (PRWH/E-DLV). J Hand Surg Eur Vol 34, 556-7 (2009).

33. Kristiansen, T.K., Ryaby, J.P., McCabe, J., Frey, J.J. \& Roe, L.R. Accelerated healing of distal radial fractures with the use of specific low-intensity ultrasound. A multicenter, prospective, randomized, double-blind, placebo-controlled study. J Bone Joint Surg Am 79, 961-73 (1997).

34. Aspenberg, P. \& Sandberg, O. Distal radial fractures heal by direct woven bone formation. Acta Orthop 84, 297-300 (2013).

35. Uhthoff, H.K. \& Rahn, B.A. Healing patterns of metaphyseal fractures. Clin Orthop Relat Res, 295-303 (1981).

36. Sevitt, $\mathrm{S}$. The healing of fractures of the lower end of the radius. A histological and angiographic study. J Bone Joint Surg Br 53, 519-31 (1971).

37. Tanck, E., Homminga, J., van Lenthe, G.H. \& Huiskes, R. Increase in bone volume fraction precedes architectural adaptation in growing bone. Bone 28, 650-4 (2001).

38. Groll, O., Lochmuller, E.M., Bachmeier, M. Willnecker, J. \& Eckstein, F. Precision and intersite correlation of bone densitometry at the radius, tibia and femur with peripheral quantitative CT. Skeletal radiology 28, 696702 (1999). 



$$
\frac{b}{x}
$$




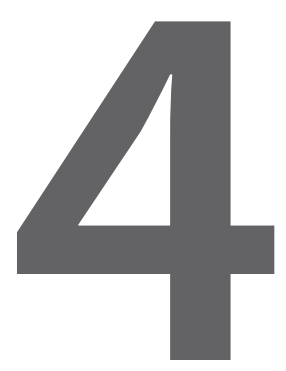

Early changes in bone density, micro-architecture, bone resorption and inflammation predict the clinical outcome twelve weeks after conservatively treated distal radius fractures

U Meyer, JJ de Jong, SG Bours, AP Keszei, JJ Arts, PRBrink, PP Menheere, TA van Geel, B van Rietbergen, JP van den Bergh, PP Geusens, PC Willems 


\section{Abstract}

\section{Background}

Fracture healing is an active process with early changes in bone and inflammation. We performed an exploratory study evaluating the association between early changes in densitometric, structural, biomechanical, and biochemical bone parameters during the first weeks of fracture healing and wrist-specific pain and disability at twelve weeks in postmenopausal women with a conservatively treated distal radius fracture.

\section{Methods}

Eighteen patients (aged $64 \pm 8$ years) were evaluated at one to two and three to four weeks post-fracture, using high-resolution peripheral quantitative computed tomography (HR-pQCT), micro finite element analysis, serum procollagen type-I Nterminal propeptide (PINP), carboxy-terminal telopeptide of type I collagen (ICTP), and high-sensitive C-reactive protein (hsCRP). After twelve weeks, patients rated their pain and disability using Patient Rated Wrist Evaluation (PRWE) questionnaire. Additionally, Quick Disability of the Arm Shoulder and Hand (QuickDASH) questionnaire and active wrist range of motion was evaluated. Linear regression models were used to study the relationship between changes in bone parameters and in hsCRP from visit one to two and PRWE score after twelve weeks.

\section{Results}

A lower PRWE outcome, indicating better outcome, was significantly related to an early increase in trabecular bone mineral density (BMD) $(\beta=-0.96[95 \% \mathrm{Cl}-1.75$ to -0.16$]$, $\left.\mathrm{R}^{2}=0.37\right)$, in torsional stiffness $\left(\beta=-0.14[95 \% \mathrm{Cl}-0.28\right.$ to -0.004$\left.], \mathrm{R}^{2}=0.31\right)$, and to an early decrease in trabecular separation $\left(\beta=209[95 \% \mathrm{Cl} 15\right.$ to 402$\left.], \mathrm{R}^{2}=0.33\right)$ and in ICTP $(\beta=12.1$ [95\% Cl 0.0 to 24.1$\left.], R^{2}=0.34\right)$. Similar results were found for QuickDASH. Higher total dorsal and palmar flexion range of motion was significantly related to early increase in hsCRP ( $\beta=9.62$ [95\% Cl 3.90 to 15.34$\left.], R^{2}=0.52\right)$.

\section{Conclusion}

This exploratory study indicates that the assessment of early changes in trabecular $B M D$, trabecular separation, calculated torsional stiffness, bone resorption marker ICTP, and hsCRP after a distal radius fracture provides valuable information regarding the twelve-week clinical outcome in terms of pain, disability, and range of motion and validates its use in studies on the process of early fracture healing. 


\section{Introduction}

Distal radius fractures are among the most common fractures in elderly.1 Although these fractures have a limited impact on general health-related quality of life, ${ }^{2,3}$ long-term complications are more commonly reported when using questionnaires especially developed to evaluate pain and function of the wrist: Persisting pain, stiffness, weakness of the wrist and signs of the complex regional pain syndrome are the most mentioned complications that limit the patient's ability to manage daily life activities, such as household work or personal care. ${ }^{4-8}$

Considering the high variability in patient-rated pain and disability, ${ }^{9}$ it would be of great clinical value to know to what degree specific bone parameters can predict long-term clinical outcome after a fracture. In studies examining baseline predictors for clinical outcome, patient factors, injury patterns, and fracture-related characteristics played only a limited role in predicting the clinical outcome after a fracture. ${ }^{10-13}$ To our knowledge, there is no study available that evaluated longterm outcome in terms of pain and disability in relation to early changes in bone mineral density (BMD), local bone architecture, and serum bone markers and inflammation during fracture healing. Fracture healing is a complex process in which inflammation and bone remodeling are intensively involved. We, therefore, hypothesized that early changes in these mechanisms would predict clinical outcome at longer term. If so, influencing these changes early in the process of fracture healing could influence the clinical outcome.

The recent development of high-resolution peripheral quantitative computed tomography (HRpQCT) allows in vivo assessment of 3D bone density and bone micro-architecture of the distal radius and calculation of biomechanical properties of the bone using micro finite element analysis $(\mu \mathrm{FEA}) .^{14,15}$ Therefore, the aim of this exploratory study was to assess the association between early fracture healing related changes in bone mineral density, bone micro-architecture, calculated biomechanical properties, and serum markers of bone turnover and inflammation with wrist-specific pain and disability at twelve weeks post-fracture in postmenopausal women with a stable, conservatively treated distal radius fracture. 


\section{Materials and Methods}

\section{Design and study population}

Twenty women aged 50 years or older that presented with a stable distal radius fracture at the departments of Orthopedic surgery and Traumatology of the Maastricht University Medical Center were included in this study. All fractures were conservatively treated by cast immobilization, if necessary after manual reduction. Exclusion criteria were a history of previous fracture at the fractured side; known systemic or metabolic disorders leading to progressive bone deterioration; use of glucocorticoids; presence of an active inflammatory disease; presence of an active or suspected infection; malignancy in the last twelve months pre-fracture. Patients were followed over a period of twelve weeks at four outpatient visits: At one to two weeks (visit one), three to four weeks (visit two), six to eight weeks (visit three) and twelve weeks (visit four) post-fracture. The protocol (registration number NTR3821) was approved by the Medical Ethics Committee of the Maastricht University Medical Center, and all patients provided written informed consent prior to participation.

\section{Clinical outcome measures}

At visit four, twelve weeks after the fracture, patients rated their pain and disability using validated Dutch versions of the Patient Rated Wrist Evaluation (PRWE) questionnaire. PRWE was chosen as the primary outcome measure because it is a simple and brief tool that was especially developed for evaluating the outcome in patients with distal radius fractures. It has previously been shown to be a reliable and valid measurement tool after distal radius fractures. ${ }^{16,17}$ The questionnaire consists of a pain subscale (five items, contributing to $50 \%$ to the total score) and a disability subscale (ten items, $50 \%$ of total score). The total PRWE score ranged from 0 (no pain/no difficulty) to 100 (worst pain/disability).

In addition, we applied the Quick Disabilities of the Arm Should and Hand (QuickDASH) questionnaire, which is the most widely used questionnaire for patients with general upper-extremity problems. It has been validated in patients with any or multiple disorders of the upper limb. ${ }^{18-21}$ QuickDASH uses eleven items to measure physical function and symptoms on a 0 to 100 scale with higher scores representing greater disability. Further, patients were asked to rate their pain on a visual analogue scale (VAS) ranging from 0 (no pain) to 100 (most severe pain). Active range of motion was measured at the fractured side for total wrist dorsal and palmar flexion and total ulnar and radial deviation. 


\section{HRpQCT measurements and micro finite element analysis ( $\mu$ FEA)}

At visit one (one to two weeks post-fracture) and visit two (three to four weeks postfracture), the fractured radius was scanned by HRpQCT (XtremeCT, Scanco Medical AG, Bruttisellen, Switzerland) using clinical in vivo settings by the manufacturer. The region of interest was based on anteroposterior (AP) and lateral radiographs of the fractured radius, in which the proximal edge of the lunate was used as reference. The scan length was set to $18 \mathrm{~mm}$ (Figure 1), which means that this is twice the scan length of the standard protocol. This doubling was needed to make sure that the complete fracture was scanned. With an isotropic voxel size of $82 \mu \mathrm{m}$, each HRpQCT measurement therefore resulted in 220 parallel CT slices with a scan time of 5.6 minutes and a radiation of $<6 \mathrm{mSv}$. Because the patient's forearm was fixed in a cast, the forearm with the cast was placed in a custom cylindrical carbon holder with an inflatable cushion (Pearltec AG, Schlieren, Switzerland) to minimize patient motion. Scan quality was graded according to Pialat et al ${ }^{22}$, a five-grade scheme ranging from grade 1 (no motion artifacts) to grade 5 (severe motion artifacts). Scans with insufficient quality (i.e. grade 4 or 5 ) were repeated.

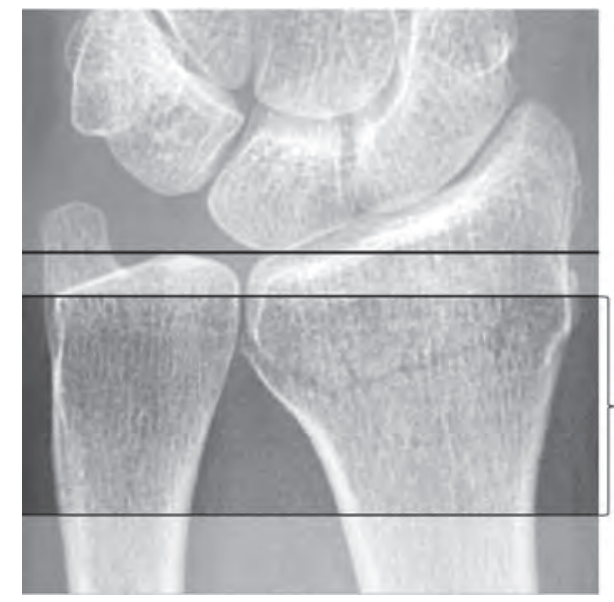

Reference line: os lunate

Region of interest: $18 \mathrm{~mm} / 220$ slices

Figure 1 Representative region of interest on the HRpQCT image of the distal radius. The proximal edge of the lunate was used as reference line. A scan length of $18 \mathrm{~mm}$ (220 sclices) was selected to make sure that the complete fracture was scanned.

The HRpQCT images were evaluated using the standard patient evaluation protocol provided by the manufacturer. ${ }^{23}$ In short, the cortical and trabecular region were separated first using a semi-automated contouring scheme in which the periosteal boundary surface of the radius was derived. ${ }^{24}$ After contouring, a Laplace-Hamming filter (epsilon 0.5 and cut-off frequency 0.4 ) followed by normalization (range 0 to 1000) and global thresholding (threshold 400), was used 
in order to extract the voxels that represented mineralized bone and to create the segmented images. The following bone density parameters were calculated from the images: volumetric bone mineral density $(\mathrm{mgHA} / \mathrm{cm} 3)$ was assessed for the total region (Dtot), and the trabecular (Dtrab) and cortical region (Dcort) separately. The micro-architectural parameters that were used in this study were: the trabecular number (Tb.N; 1/mm), thickness (Tb.Th; $\mathrm{mm}$ ) and separation (Tb.Sp; $\mathrm{mm}$ ), which are all determined using a 3D ridge extraction method ${ }^{25}$ and standard morphological relations. Bone geometry was expressed by the cortical thickness (Ct.Th; mm), which was calculated by dividing the cortical volume by the outer cortical surface.

$\mu \mathrm{FE}$ models were created from the segmented HRpQCT images. Each voxel that represents bone tissue was converted into a brick element of the same size. In the standard $\mu \mathrm{FE}$ approach usually used in $\mathrm{HRpQCT},{ }^{14}$ equal properties are assigned to every bone tissue element. Whereas this approach has been well validated and successfully applied in clinical studies, its application to fractured bone is not trivial. The use of segmented images limits the models to only represent the mineralized phase. In particular in the early stages of fracture healing this approach might to be inaccurate because it does not account for newly formed low mineralized bone. Therefore, we additionally applied a more sophisticated approach, earlier described by Shefelbine and colleagues, ${ }^{26}$ that distinguishes between three different tissue types (soft tissue, low mineralized tissue and high mineralized tissue) with stiffness properties of the elements depending on its material composition. This approach has been validated for healing bone. ${ }^{26}$ In the resulting $\mu \mathrm{FE}$ model soft tissue was assigned a constant modulus of $50 \mathrm{MPa}$ whereas for mineralized bone a Young's modulus ranging from 5000 to $15000 \mathrm{MPa}$ results, depending on the material composition of the voxel. A Poisson's ratio of 0.3 was assigned to all elements and all materials were assumed to be linear elastic and isotropic. For the standard $\mu \mathrm{FE}$ approach, a procedure similar to that described in earlier studies was used and all elements were assigned a Young's modulus of $10 \mathrm{GPa}$ and a Poissons ratio of 0.3. ${ }^{14,27}$ By subsequently applying different load cases, clinical relevant loading scenarios were simulated and the associated stiffness was chosen as outcome variable. The first load case represented a 'high friction' compression test with a prescribed displacement in the axial direction of $1 \%$ of the total length, from which the compression stiffness (Scomp; kN/mm) was calculated. The second load case represented a prescribed rotation of $0.01 \mathrm{rad}$ around the longitudinal axis applied to the surface perpendicular to this axis from which the torsional stiffness (Stors; $\mathrm{kNmm} / \mathrm{rad}$ ) was calculated. A third and fourth load case represented a prescribed rotation of $0.01 \mathrm{rad}$ applied around the sagittal and transversal axes respectively, onto the surfaces perpendicular to each axis, thus inducing a state of pure bending 
in two directions, from which the bending stiffness in each direction (sagittal and transversal) was calculated. Because the orientation of the patient's forearm was not the same during all visits, a quadratic mean bending stiffness was calculated (Sbend; $\mathrm{kNmm}^{2} / \mathrm{rad}$ ) from the sagittal and transversal bending stiffness.

In addition to HRpQCT, and according to the Dutch standard care when a patient over the age of 50 years comes to clinic with a fracture, BMD T-score at the femoral neck was assessed by dual-energy X-ray absorptiometry (DXA; Hologic Discovery A; Hologic Inc., Waltham, MA, USA). BMD T-score was assessed once between four and eight weeks post-fracture, or was taken from a previous DXA scan if the patients already had had a DXA scan in the last year prior to the fracture.

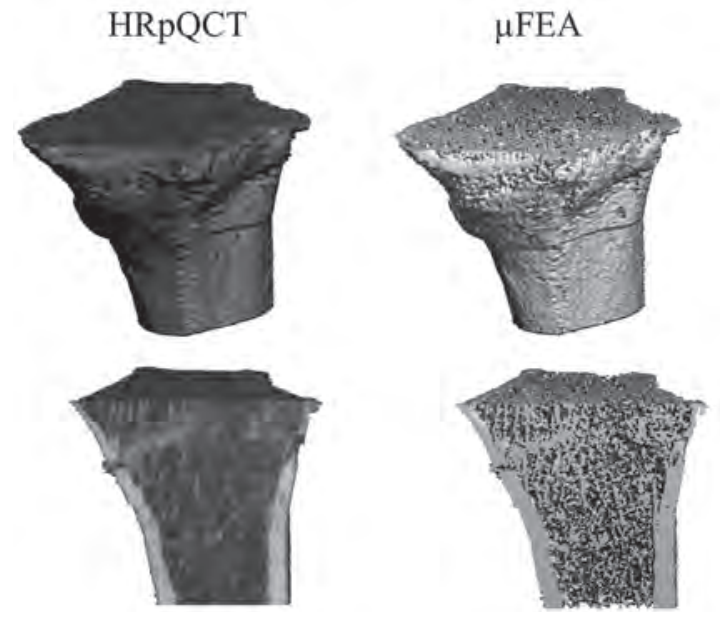

Figure 2 Representative image of an HRpQCT and a FEA model.

\section{Serum markers of bone turnover and inflammation, and vitamin D}

At visit one (one to two weeks post-fracture) and visit two (three to four weeks post-fracture), venous blood samples were collected by puncture of the antecubital vein. Serum was separated from the bloodclot within 20 minutes and freezed to $-20^{\circ} \mathrm{C}$ until analysis. Serum bone markers representing bone resorption (carboxyterminal telopeptide of type I collagen [ICTP]) and bone formation (procollagen type-I N-terminal propeptide [PINP]) were assessed by radioimmunoassay (UniQ PINP/ICTP; Orion Diagnostics, Espoo, Finland) in a certified laboratory. Additionally, fracture-induced inflammation was assessed by high-sensitive C-reactive protein (hsCRP) (BN ProSpec system; Siemens/Dade Behring, Liederbach, Germany). An additional blood sample was taken at visit two to analyze 25-hydroxyvitamin D $(25(\mathrm{OH}) \mathrm{D})$ by chemiluminescent immunoassay (IDS-iSYS Immunodiagnostic Systems GmbH, Frankfurt, Germany). 


\section{Statistical analysis}

Patient's demographic characteristics, bone parameters and clinical outcomes at twelve weeks post-fracture were reported in means and their standard deviations. Changes in bone parameters between visit one and visit two were tested using a paired samples Student's t-test. A linear regression was used to determine if the clinical outcome at twelve weeks was related to patients' or fractures'characteristics, such as fracture type (intra- versus extra-articular, comminuted versus noncomminuted, reduced versus non-reduced), fracture side (dominant versus nondominant), DXA T-score at femoral neck, or serum vitamin D level $(\geq 75 \mathrm{nmol} / \mathrm{L}$ versus $<75 \mathrm{nmol} / \mathrm{L})$. The relationship between early changes in bone parameters and twelve-week clinical outcomes was calculated using a linear regression with the clinical outcome at twelve weeks post-fracture as outcome, and the change in bone parameters between visit one and visit two as explanatory variable. The model was adjusted for the number of days between visit one and visit two. Because adjusting the model for baseline bone values (i.e. bone parameters at visit one) did not improve the model according to the Akaike information criterion, we did not include baseline values in the model. In a second step, we additionally adjusted the model for fracture type (intra- versus extra-articular, comminuted versus non-comminuted, reduced versus non-reduced), fracture side (dominant versus non-dominant), DXA T-score at femoral neck, or serum 25(OH)D level ( $\geq 75$ $\mathrm{nmol} / \mathrm{L}$ versus $<75 \mathrm{nmol} / \mathrm{L}$ ) to test whether these factors influenced the model. All analyses were performed using Stata 11.0 (StataCorp, College Station, TX, USA) and the significance level was set to $\alpha=0.05$.

\section{Results}

\section{Patient's characteristics}

From the twenty patients who were initially included in the study, one patient declined participation at the first visit and another patient at the second visit. Thus, eighteen women $(64.3 \pm 8.4$ years $)$ completed the study. The characteristics of these eighteen patients, including their fracture characteristics are listed in Table 1. Mean DXA T-score at femoral neck was -2.2 (0.9) with seven (44\%) osteoporotic, and eight (50\%) osteopenic women. Two patients had no DXA data. Serum 25-hydroxyvitamin D levels were 62.8 (29.3) $\mathrm{nmol} / \mathrm{L}$, with ten (46\%) patients having values below $75 \mathrm{nmol} / \mathrm{L}$. The clinical outcome in terms of pain and disability, as assessed at visit four (85.4 (range 81 to 91) days post-fracture), are also presented in Table 1. According to the PRWE classification of MacDermid et al., ${ }^{9}$ five (28\%) patients reported to experience no or minimal pain and disability, whereas three 
(17\%) patients had mild, five (28\%) patients moderate, five (28\%) patients severe, and none of the patient very severe pain or disability, respectively, twelve weeks after the fracture. In one patient, VAS at twelve weeks post-fracture is missing, and two patients the range of motion test could not have been performed. HRpQCT measurements were performed 9.1 (4 to 16) days (visit one) and 23.8 (18 to 29) days after the fracture (visit two) with the corresponding values shown in Table 2. 36\% of the scans had to be repeated due to motion artifacts. Even if after repetition, five scans were still of insufficient quality, they were included into analysis. A representative image of a HRPQCT and a FEA model is given in Figure 2. One patient has missing ICTP, PINP and hsCRP data.

\section{Relation between baseline characteristics and clinical outcome twelve weeks post-fracture}

PRWE total score or QuickDASH score twelve weeks post-fracture were not related to baseline age, or fracture side (dominant versus non-dominant side) and type (intra-articular, comminuted, or reduced fracture), DXA T-score or 25(OH)D. VAS was higher in patients with a fracture at the dominant side (33.0 [95\% confidence interval: 3.4 to 62.6] VAS counts; $p=0.031$; adjusted $R^{2}=0.23$ ) compared to the non-dominant side. Lower palmar flexion range of motion was found after an intra-articular fracture ( -23.3 [ -46 to -0.7$]$ angular degrees; $p=0.044 ; R^{2}=0.21$ ), and lower total ulnar and radial deviation range of motion after a reduced fracture $\left(-17.8[-233.7\right.$ to -1.8$]$ angular degrees; $\left.p=0.032 ; R^{2}=0.24\right)$. 


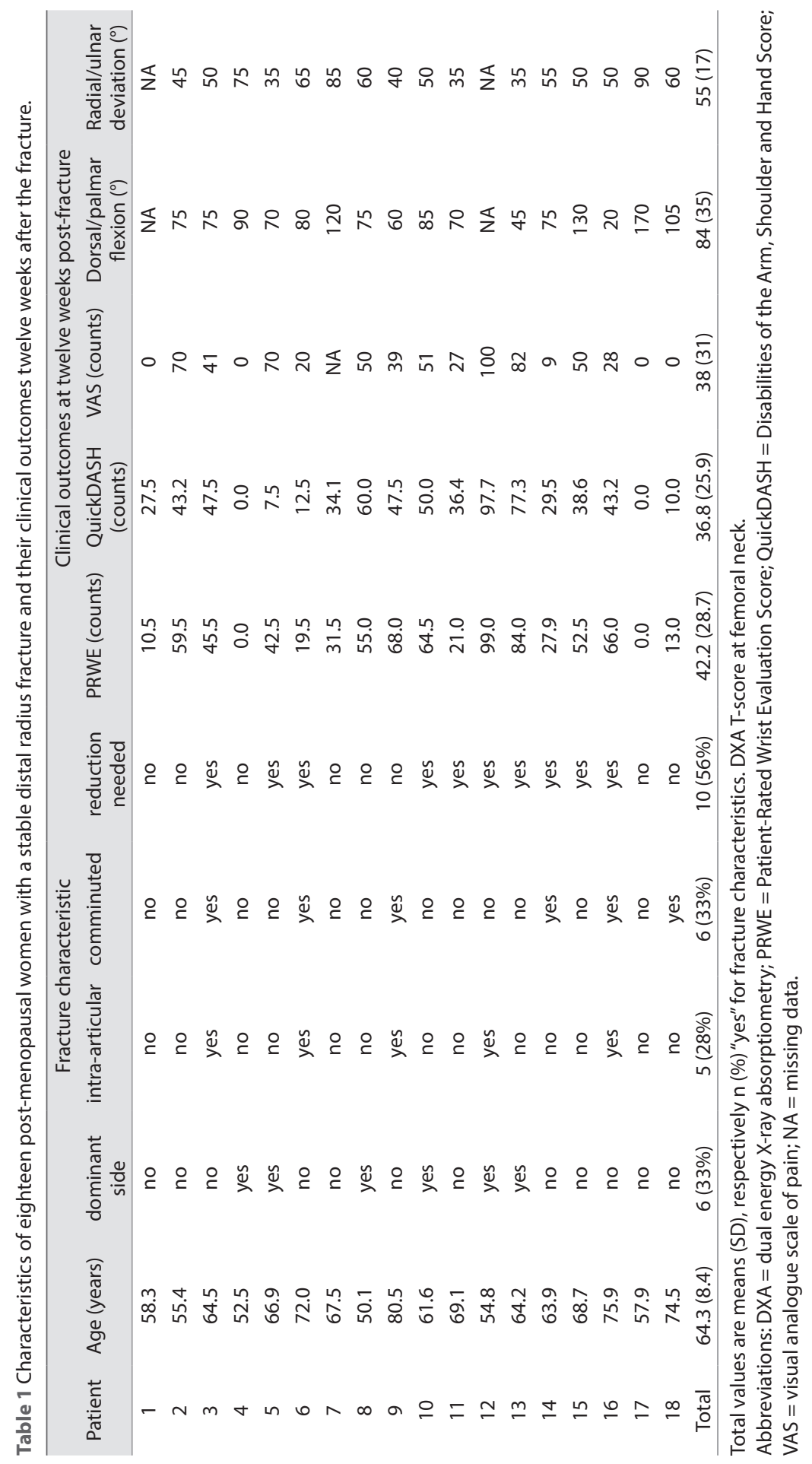


A

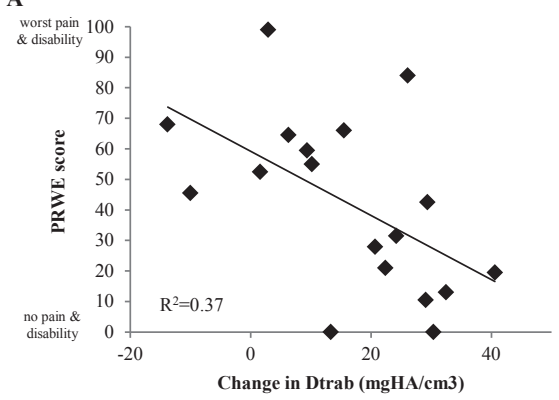

C

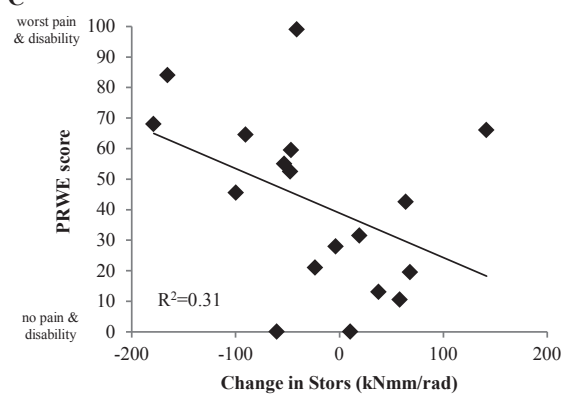

B

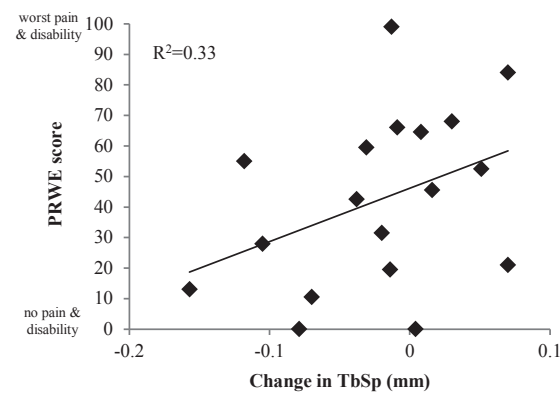

D

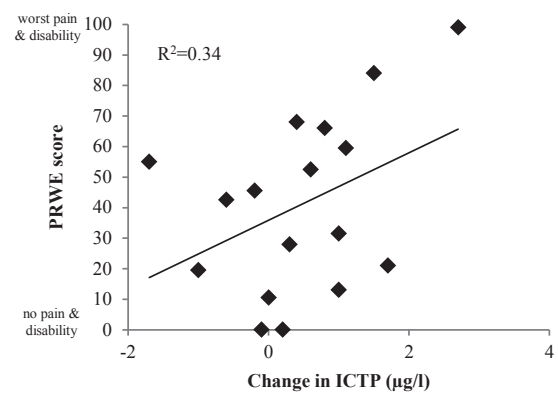

Figure 3 Relation between the change in (A) trabecular density (Dtrab), (B) trabecular separation (TbSp), (C) torsional stiffness (Stors), (D) bone resorption (carboxy-terminal telopeptide of type I collagen [ICTP]) between visit one (one to two weeks post-fracture) and visit two (three to four weeks post-fracture) and patient-rated pain and disability (PRWE score) twelve weeks after a distal radius fracture.

\section{PRWE score}

The relationship of PRWE at twelve weeks and the early changes in bone parameters is shown in Table 3 and Figure 3. Higher early increases in Dtrab were significantly related to lower, thus better, PRWE scores at twelve weeks post-fracture ( $p=$ 0.022; adjusted $R^{2}=0.37$ ). For the other density parameters (Dcort and Dtot), the relationship was not significant.

On micro-architectural level, lower PRWE was related to higher decreases in Tb.Sp ( $p=0.037 ; R^{2}=0.33$ ), but not to changes in Tb.N or Tb.Th.

On the biomechanical level, lower PRWE score was related to higher increases in Stors $\left(p=0.045 ; R^{2}=0.31\right)$, but not to changes in Scomp nor for Sbend. When applying the Shefelbine method, the relation between Stors and PRWE was -0.134 $\left(-0.290\right.$ to $\left.0.022 ; p=0.086 ; R^{2}=0.26\right)$. 
Table 2 Bone parameters at the fracture location assessed by HRpQCT, calculated biomechanical parameters and serum bone markers at one to two weeks (visit 1), three to four weeks (visit 2) and the change between visit 1 and 2 , after a stable distal radius fracture.

\begin{tabular}{lccc}
\hline & $\begin{array}{c}\text { Visit } 1 \\
\text { (one to two wks } \\
\text { post-fx) }\end{array}$ & $\begin{array}{c}\text { Visit 2 } \\
\text { (three to four wks } \\
\text { post-fx) }\end{array}$ & $\begin{array}{c}\text { Change between } \\
\text { visit } 1 \text { and } 2\end{array}$ \\
\hline $\begin{array}{l}\text { Density parameters } \\
\text { Dtot }(\mathrm{mgHA} / \mathrm{cm} 3)\end{array}$ & $232.2(54.8)$ & $243.4(50.4)$ & $11.2(1.8 \text { to } 20.6)^{\mathrm{a}}$ \\
Dcort $(\mathrm{mgHA} / \mathrm{cm} 3)$ & $648.1(123.4)$ & $629.3(113.9)$ & $-18.8(-32.7 \text { to }-4.8)^{\mathrm{a}}$ \\
Dtrab $(\mathrm{mgHA} / \mathrm{cm} 3)$ & $144.8(26)$ & $160.9(29)$ & $16.2(8.7 \text { to } 23.6)^{\mathrm{a}}$ \\
Micro-structural parameters & & & \\
Ct.Th $(\mathrm{mm})$ & $0.408(0.216)$ & $0.4(0.193)$ & $-0.008(-0.039$ to 0.022$)$ \\
Tb.N $(\mathrm{mm}-1)$ & $1.995(0.455)$ & $2.029(0.423)$ & $0.034(-0.064$ to 0.131$)$ \\
Tb.Th $(\mathrm{mm})$ & $0.063(0.015)$ & $0.068(0.013)$ & $0.005(0.001 \text { to } 0.009)^{\mathrm{a}}$ \\
Tb.Sp $(\mathrm{mm})$ & $0.468(0.131)$ & $0.445(0.099)$ & $-0.023(-0.054$ to 0.009$)$ \\
Biomechanical parameters (standard protocol) & & \\
Scomp $(\mathrm{kN} / \mathrm{mm})$ & $14.8(7.2)$ & $13.0(7.8)$ & $-1.8(-3.6$ to 0.03$)$ \\
Stors $(\mathrm{kNmm} / \mathrm{rad})$ & $298(135)$ & $272(120)$ & $-26(-69$ to 18$)$ \\
Sbend $(\mathrm{kNmm} / \mathrm{rad})$ & $430(227)$ & $379(213)$ & $-51(-127$ to 26$)$ \\
Serum bone markers & & & \\
ICTP $(\mu \mathrm{m} / \mathrm{l})$ & $3.6(1.1)$ & $4.1(1.1)$ & $0.5(-0.1$ to 1.0$)$ \\
PINP $(\mu \mathrm{gg} / \mathrm{l})$ & $48.9(25.7)$ & $65.9(39.1)$ & $12.9(-3.7$ to 29.5$)$ \\
hsCRP $(\mathrm{mg} / \mathrm{l})$ & $3.8(6.3)$ & $2.6(4.5)$ & $-1.2(-2.8$ to 0.4$)$ \\
\hline
\end{tabular}

Values are expressed in means and their standard deviations for visit one and 2, and as mean $(95 \%$ confidence interval) for the change between the two visits.

Abbreviations: Dtot $=$ total bone mineral density; Dcort $=$ cortical bone mineral density; Dtrab $=$ trabecular bone mineral density; $\mathrm{Ct} . \mathrm{Th}=$ cortical thickness; $\mathrm{Tb} . \mathrm{N}=$ trabecular number; $\mathrm{Tb} . \mathrm{Th}=$ trabecular thickness; Tb.Sp = trabecular separation; Scomp $=$ compressional stiffness; Stors $=$ torsional stiffness; Sbend $=$ bending stiffness; ICTP = carboxy-terminal telopeptide of type I collagen; PINP = procollagen type-I N-terminal propeptide; hsCRP = high-sensitive C-reactive protein.

a refers to significant changes between visit 1 and 2 .

On serum bone marker level, higher PRWE was related to higher increases in ICTP ( $\left.p=0.050 ; R^{2}=0.34\right)$ but not to changes in PINP or hsCRP.

Adjusting the analysis for fracture type (intra-articular fracture, comminuted fracture, or reduced fracture) did not influence the results, however including fracture side (dominant versus non-dominant hand) into the model increased adjusted $R^{2}$ to 0.45 for the relation with early changes in Dtrab $(-1.03[-1.78$ to -0.27]; $p=0.011$ ) with higher PRWE values for patients with a fracture at the dominant side $\left(\mathrm{p}_{\mathrm{f} x \text {-side }}<0.1\right)$. The $25(\mathrm{OH}) \mathrm{D}$ levels did not influence the model, nor did the DXA T-scores.

Excluding the five patients that had one scan with insufficient quality (subsample of thirteen patients), beta-coefficients for early changes in Dtrab, Tb.Sp, Stors, and ICTP remained at the same level or became stronger. However, the associations were not significant anymore. 
Table 3 Relationship between the PRWE score at twelve weeks post-fracture as a measure for pain and disability, and the changes in bone parameters at the fracture location assessed by HRPQCT, calculated biomechanical parameters and serum bone markers between one to two weeks (visit 1) and three to four weeks (visit 2), after a stable distal radius fracture.

\begin{tabular}{|c|c|c|c|}
\hline & $\beta$-coefficient (95\% confidence interval) & $p$-value & Adj R2 \\
\hline \multicolumn{4}{|c|}{ Density parameters } \\
\hline Dtot & $-0.61(-1.28$ to 0.06$)$ & 0.073 & 0.27 \\
\hline Dcort & $-0.19(-0.68$ to 0.31$)$ & 0.438 & 0.13 \\
\hline Dtrab & $-0.96(-1.75$ to -0.16$)$ & $0.022^{\mathrm{a}}$ & 0.37 \\
\hline \multicolumn{4}{|c|}{ Micro-structural parameters } \\
\hline Ct.Th & $-91(-323$ to 140$)$ & 0.412 & 0.13 \\
\hline Tb.N & $-48.8(-116$ to 18.4$)$ & 0.142 & 0.22 \\
\hline Tb.Th & $-352(-2089$ to 1386$)$ & 0.672 & 0.10 \\
\hline Tb.Sp & 209 (15 to 402) & $0.037^{a}$ & 0.33 \\
\hline \multicolumn{4}{|c|}{ Biomechanical parameters (standard protocol) } \\
\hline Scomp & $-2.44(-6.18$ to 1.31$)$ & 0.186 & 0.19 \\
\hline Stors & $-0.145(-0.285$ to -0.004$)$ & $0.045^{\mathrm{a}}$ & 0.31 \\
\hline Sbend & $-0.064(-0.15$ to 0.022$)$ & 0.132 & 0.22 \\
\hline \multicolumn{4}{|c|}{ Serum bone markers } \\
\hline ICTP & 12.1 (0.0 to 24.1$)$ & $0.050^{\mathrm{a}}$ & 0.34 \\
\hline PINP & $0.256(-0.175$ to 0.687$)$ & 0.223 & 0.21 \\
\hline hsCRP & $-0.263(-5.02$ to 4.495$)$ & 0.907 & 0.12 \\
\hline
\end{tabular}

Abbreviations: Dtot $=$ total bone mineral density; Dcort $=$ cortical bone mineral density; Dtrab = trabecular bone mineral density; $\mathrm{Ct}$.Th = cortical thickness;Tb.N = trabecular number; Tb.Th = trabecular thickness; Tb.Sp = trabecular separation; Scomp = compressional stiffness; Stors = torsional stiffness; Sbend = bending stiffness; ICTP = carboxy-terminal telopeptide of type I collagen; PINP = procollagen type-I N-terminal propeptide; hsCRP = high-sensitive C-reactive protein; PRWE = Patient-Rated Wrist Evaluation Score.

${ }^{a}$ refers to a statistically significant relation.

\section{QuickDASH score}

QuickDASH and PRWE score twelve weeks post-fracture were highly correlated $(r=0.89)$. QuickDASH was related to changes in Dtrab $(-0.789[-1.49$ to -0.08$] ; p=$ $0.031 ; R^{2}=0.40$ ), Tb.Sp (174 [5.7 to 343]; $p=0.044 ; R^{2}=0.37$ ), ICTP (13.0 [3.4 to 22.6]; $\left.p=0.012 ; R^{2}=0.49\right)$, and Stors applying the Shefelbine method $(-0.138[-0.266$ to $-0.010] ; p=0.036 ; R^{2}=0.39$ ) (Table 3).

\section{Visual analogue scale}

VAS was highly correlated to PRWE score and QuickDASH score twelve weeks postfracture ( $r=0.88$ and $r=0.76$, respectively). VAS score was not significantly related to early changes in bone, biomechanical, or serum bone marker parameters. After correction for fracture side, VAS was significantly related to early changes in Tb.Sp (226 [26.5 to 425]; $p=0.029 ; R^{2}=0.41$ ) (Table 3 ). 


\section{Range of motion}

Range of motion at twelve weeks was significantly correlated with PRWE score, QuickDASH and VAS (all $r<-0.57$ ). The total palmar and dorsal flexion was related to early changes in hsCRP (9.62 [3.90 to 15.34]; $p=0.003 ; R^{2}=0.52$ ) (Figure 4), but range of motion did not appear to be related to changes in bone density, architecture, and biomechanical parameters. The total radial-ulnar deviation range of motion was not related to early changes in bone parameters including hsCRP. After correction for intra-articular or reduced fractures, the effects remained the same.

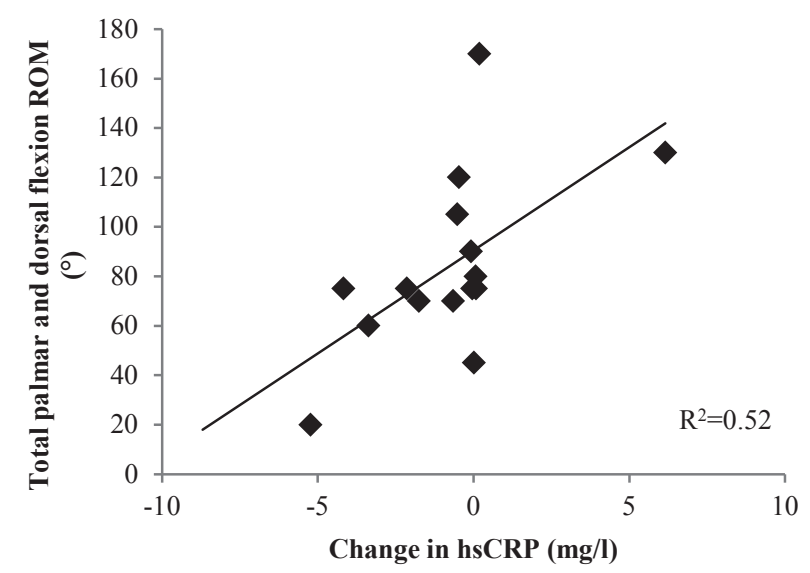

Figure 4 Relation between the change in high-sensitive C-reactive protein (hsCRP) between visit one (one to two weeks post-fracture) and visit two (three to four weeks post-fracture) and total active dorsal and palmar flexion range of motion twelve weeks after a distal radius fracture. Two patients have missing range of motion data, one patient does not have hsCRP data.

\section{Discussion}

The most striking finding in this study is that the changes in bone micro-architecture, calculated bone stiffness, bone resorption and inflammation during the first weeks of fracture healing predicted functional outcome parameters assessed at twelve weeks after a distal radius fracture. PRWE score twelve weeks after the fracture were explained for more than $30 \%$ by changes in Dtrab, Stors, Tb.Sp, and ICTP between day 9 and 23 after fracture. This indicates that early changes in bone structure and remodeling are important for clinical recovery; hence, a greater increase in Dtrab and Stors and greater decrease in bone resorption are related to a better long-term outcome for pain and function.

Each $10 \mathrm{mgHA} / \mathrm{cm}^{3}$ higher early increase in Dtrab between visit one and two was related to a lower PRWE by 9.6 counts and a lower QuickDASH score by 
7.9 counts, respectively. Since longitudinal changes of $\geq 14$ points for PRWE and QuickDASH scores are considered minimal clinically significant changes ${ }^{28}$, the wide spectrum of early changes in Dtrab ranging from -13.8 to $+40.6 \mathrm{mgHA} / \mathrm{cm}^{3}$ may be considered as clinically meaningful. Reasons for smaller initial increases or even decreases in Dtrab after a fracture, however, remain unclear. One possibility is that either these patients' healing process has already started with increased remodeling, or that the process is delayed. The latter could explain persistent or enhanced pain and disability twelve weeks post-fracture in these patients. In this respect, it would be interesting to see whether pain and disability will improve in these patients at later follow-up or whether this patient group will have substantially more persisting symptoms.

Each $1 \mathrm{mg} / \mathrm{L}$ higher early increase in ICTP was related to a higher, thus worse PRWE by 12.1 counts at twelve weeks. During fracture healing, both markers of bone formation and resorption increase and can be used to reflect the stages of fracture healing and mineralization process. ${ }^{29-34}$ It has been shown that serum bone resorption markers, such as ICTP, rise at an earlier stage than bone formation markers, indicating that during the very first weeks after a fracture, osteoclast activation-driven resorption occurs as the main process. ${ }^{31,32}$ This could be an explanation why in our study, the early increase in ICTP and not PINP was related to pain and disability at follow-up. It has been reported that patients with a delayed fracture healing tended to have higher increases in bone resorption markers in the first weeks post-fracture. ${ }^{33}$ Fracture extend and location may also increase ICTP response after a fracture with higher increases in more complex fractures, or larger fracture areas. ${ }^{32,34}$ However, more complex fractures, in our study represented by intra-articular, comminuted and/or fractures where a manual reduction was necessary, had no influence on the results.

Interestingly, early changes in hSCRP did not contribute to PRWE and QuickDASH score, suggesting that early changes in inflammation do not contribute to long-term pain and disability. In contrast, active range of motion was explained by $40 \%$ to $60 \%$ by early changes in hsCRP, indicating that early increase in inflammation resulted in better active motion at twelve weeks post-fracture. Inflammation is considered as an important primary stimulus for fracture repair. ${ }^{35,}$ ${ }^{36}$ The beneficial effect of inflammation during fracture healing is also supported by studies showing that anti-inflammatory drugs may delay fracture healing. ${ }^{36}$

On the other hand, no significant predictors of VAS were identified. Whereas PRWE and QuickDASH questionnaires both consist of questions about pain and functionality, VAS only includes information about patient-perceived pain. However, using the pain subscale of the PRWE questionnaire as outcome (data 
not shown), the results remained the same as for the total PRWE score. A possible explanation for the different results when using VAS could be that the VAS evaluates pain intensity, but not frequency, whereas PRWE also included frequency-related questions. Additionally, perceived pain is determined by many more factors than the healing process of the mineralized phase of bone after a fracture as assessed by HRpQCT only. Further, differences in perceived pain could have influenced the clinical outcome at twelve weeks, and also the early changes in bone parameters, as patients with a higher pain tolerance might use their fractured arm more extensively. VAS score at the contra-lateral side could be an indicator of general pain tolerance. However, it did not influence our results (data not shown).

These results suggest that the integrated process of inflammation, regeneration, and remodeling that occurs during the early stages of fracture healing can be studied by the assessment of hsCRP, bone resorption markers and bone structure by HRpQCT in order to predict clinical outcome three months after a stable distal radius fracture.

More than half of all patients (56\%) reported they still experienced moderate to very severe pain and disability twelve weeks after the fracture. A painless, full restoration of hand functionality is seen as the major goal after a distal radius fracture. ${ }^{37}$ Knowing more about fracture related predictors for clinical outcomes is important to identify patients at risk for having long-term complications. Once they are identified, it could become possible to treat them. Interestingly, patient and injury related characteristics (as our data also confirmed) play only a small role in predicting the clinical outcome after a fracture. ${ }^{10,11}$. While injury compensation, ${ }^{10,}$ ${ }^{11}$ patients' education level, ${ }^{10,11}$ the presence of other medical problems, ${ }^{11}$ and prereductional radial shortening ${ }^{10}$ together explained $17 \%$ of the one-year ${ }^{11}$ and $25 \%$ of the six-month ${ }^{10}$ PRWE score, respectively, it is less consistent whether more injury related parameters (i.e. initial displacement of the fracture) predict clinical outcomes after a distal radius fracture ${ }^{13,38}$. To our knowledge, no previous study evaluated the predictive values of the fracture healing process itself on pain and disability after a distal radius fracture. However, the reason why some patients show a greater increase in Dtrab or decrease in ICTP, respectively, and whether this will lead to a more rapid consolidation and thus improved clinical recovery, remains unclear. The amount of structural changes and bone marker response could depend on the complexity of the fracture. However, in our study including conservatively treated fractures only, the associations between early changes in bone parameters and functional outcomes at twelve weeks were independent of articular involvement (intra- versus extra-articular fracture), comminution (comminuted versus noncomminuted fracture), and fracture reduction. Furthermore, the correlations 
between early bone changes and pain and disability were independent of patient's vitamin D level or DXA T-score.

Our study has several strengths. Compared to other studies evaluating the clinical outcomes after distal radius fractures, the patients and fracture type in the current study are more homogeneous. We only included post-menopausal women with a stable, conservatively treated distal radius fracture. Of course, this reduces generalization of the results to other, more complex fractures, but it gives a clearer picture of what happens in conservatively treated fractures in mainly osteopenic or osteoporotic women. Further, this is the first study to our knowledge using HRpQCT measures for the evaluation of the fracture healing process and its relationship to the clinical outcomes. The HRpQCT measurements as well as the $\mu$ FEA were obtained using the standard evaluation protocols provided by the manufacturer. Further, we used well-validated and widely used questionnaires that are recommended when evaluating clinical outcomes of distal radius fractures ${ }^{37}$.

Apart from these strengths, some limitations have to be mentioned. With eighteen patients, statistical power is limited, and therefore, our preliminary findings should be confirmed in larger studies. Because of this limited sample size, it was not feasible to analyze all bone parameters simultaneously to quantify the relative importance of predictors. Further, one should be careful with the interpretation of the derived structural parameters, such as the trabecular thickness, because the presence of woven bone between the trabeculae might affect the calculated of these parameters in an unexpected way. Additionally, our 'baseline' value was assessed 9.1 days after the fracture during the first follow-up visit, which does not allow us to set these early changes in relation to bone density and structure before nor immediately after the fracture. Also, clinical outcomes were assessed at three months post-fracture, whereas it would be interesting to also know the clinical outcomes at a longer period post-fracture. Once our results are confirmed in a larger population, future research should focus on whether certain interventions during fracture healing, for example an immediate vitamin D or calcium supplementation, could improve fracture healing on a structural level and thereby maybe reduce patients' long-term pain and disability. In order to identify patients at risk, further research should also test whether HRpQCT is needed to evaluate these early changes in Dtrab after a fracture, or if a clinical QCT with poorer resolution, and/or other serum markers have the same power to predict clinical outcomes.

In conclusion, our explorative study showed that early changes in trabecular BMD, calculated torsional stiffness, bone resorption marker ICTP and hsCRP after a 
stable distal radius fracture are related with pain and disability after twelve weeks. If larger studies confirm these results, this suggests that assessment of these early changes during fracture healing could provide valuable information regarding the long-term clinical outcome of these fractures.

\section{Acknowledgements}

The authors thank Liesbeth Jutten, Margareth Winants, and Kevin Görritz for their help in patient recruitment. The study (Dutch trial register NTR3821) was supported by The Weijerhorst Foundation (WH-2). UM received a fellowship for prospective researchers by the Swiss National Science Foundation for this project.

Author's role: Study design: CA, PB, PM, BvR, JvdB, PG, PW. Study conduct: JdJ, SB, CA, TvG, JvdB, PG, PW. Data collection: JdJ, SB, CA, PM, PW. Data analysis: UM, JdJ, BvR. Data interpretation: UM, JdJ, CA, JvdB, PG, PW. Drafting manuscript: UM, JdJ. Revising manuscript content: SB, CA, PB, PM, TvG, BvR, JvdB, PG, PW. Approving final version of manuscript: UM, JdJ, SB, CA, PB, PM, TvG, BvR, JvdB, PG, PW. UM takes responsibility for the integrity of the data analysis. 


\section{References}

1. Johnell, O. \& Kanis, J.A. An estimate of the worldwide prevalence and disability associated with osteoporotic fractures. Osteoporosis International 17, 1726-33 (2006).

2. Brenneman, S.K., Barrett-Connor, E., Sajjan, S., Markson, L.E. \& Siris, E.S. Impact of recent fracture on health-related quality of life in postmenopausal women. J Bone Miner Res 21, 809-16 (2006).

3. Adachi, J.D. et al. The association between osteoporotic fractures and health-related quality of life as measured by the Health Utilities Index in the Canadian Multicentre Osteoporosis Study (CaMos). Osteoporos Int 14, 895-904 (2003).

4. Bialocerkowski, A.E. Difficulties associated with wrist disorders - a qualitative study. Clinical Rehabilitation 16, 429-440 (2002).

5. Atkins, R.M., Duckworth, T. \& Kanis, J.A. Features of algodystrophy after Colles' fracture. J Bone Joint Surg Br 72, 105-10 (1990).

6. Geusens, P. \& Santen, M. Algodystrophy. Baillieres Best Pract Res Clin Rheumatol 14, 499-513 (2000).

7. Field, J. \& Atkins, R.M. Algodystrophy is an early complication of Colles' fracture. What are the implications? Journal of Hand Surgery. British Volume 22, 178-82 (1997).

8. Bickerstaff, D.R. \& Kanis, J.A. Algodystrophy: an under-recognized complication of minor trauma. Br J Rheumatol 33, 240-8 (1994).

9. MacDermid, J.C., Roth, J.H. \& Richards, R.S. Pain and disability reported in the year following a distal radius fracture: a cohort study. BMC Musculoskelet Disord 4, 24 (2003).

10. MacDermid, J.C., Donner, A., Richards, R.S. \& Roth, J.H. Patient versus injury factors as predictors of pain and disability six months after a distal radius fracture. $J$ Clin Epidemiol 55, 849-54 (2002).

11. Grewal, R., MacDermid, J.C., Pope, J. \& Chesworth, B.M. Baseline predictors of pain and disability one year following extraarticular distal radius fractures. $\operatorname{Hand}(N Y) \mathbf{2}$, 104-11 (2007)

12. Chung, K.C., Kotsis, S.V. \& Kim, H.M Predictors of functional outcomes after surgical treatment of distal radius fractures. J Hand Surg Am 32, 76-83 (2007).
13. Karnezis, I.A. \& Fragkiadakis, E.G. Association between objective clinical variables and patient-rated disability of the wrist. J Bone Joint Surg Br 84, 967-70 (2002).

14. Pistoia, W. et al. Estimation of distal radius failure load with micro-finite element analysis models based on three-dimensional peripheral quantitative computed tomography images. Bone 30, 842-8 (2002).

15. Vilayphiou, N. et al. Finite element analysis performed on radius and tibia HRpQCT images and fragility fractures at all sites in postmenopausal women. Bone 46, 1030-7 (2010).

16. MacDermid, J.C. Development of a scale for patient rating of wrist pain and disability. $J$ Hand Ther 9, 178-83 (1996).

17. Brink, S.M., Voskamp, E.G., Houpt, P. \& Emmelot, C.H. Psychometric properties of the Patient Rated Wrist/Hand Evaluation Dutch Language Version (PRWH/E-DLV). J Hand Surg Eur Vol 34, 556-7 (2009).

18. Beaton, D.E., Wright, J.G. \& Katz, J.N Development of the QuickDASH: comparison of three item-reduction approaches. J Bone Joint Surg Am 87, 1038-46 (2005).

19. Beaton, D.E. et al. Measuring the whole or the parts? Validity, reliability, and responsiveness of the Disabilities of the Arm, Shoulder and Hand outcome measure in different regions of the upper extremity. J Hand Ther 14, 12846 (2001).

20. Gummesson, C., Atroshi, I. \& Ekdahl, C. The disabilities of the arm, shoulder and hand (DASH) outcome questionnaire: longitudinal construct validity and measuring selfrated health change after surgery. BMC Musculoskelet Disord 4, 11 (2003).

21. Veehof, M.M., Sleegers, E.J., van Veldhoven, N.H., Schuurman, A.H. \& van Meeteren, N.L. Psychometric qualities of the Dutch language version of the Disabilities of the Arm, Shoulder, and Hand questionnaire (DASH-DLV). J Hand Ther 15, 347-54 (2002).

22. Pialat, J., Burghardt, A.J., Sode, M., Link, T.M. \& Majumdar, S. Visual grading of motion induced image degradation in high-resolution peripheral computed tomography: Impact of image quality on measures of bone density and microarchitecture. Bone 50, 111-8 (2012).

23. Laib, A., Hauselmann, H.J. \& Ruegsegger, P. In vivo high-resolution $3 \mathrm{D}-\mathrm{QCT}$ of the human forearm. Technol Health Care 6, 329-37 (1998). 
24. Laib, A. \& Ruegsegger, P. Comparison of structure extraction methods for in vivo trabecular bone measurements. Comput Med Imaging Graph 23, 69-74 (1999).

25. Hildebrand, T., Laib, A., Muller, R., Dequeker, J. \& Ruegsegger, P. Direct three-dimensional morphometric analysis of human cancellous bone: microstructural data from spine, femur, iliac crest, and calcaneus. J Bone Miner Res 14, 1167-74 (1999).

26. Shefelbine, S.J. et al. Prediction of fracture callus mechanical properties using micro-CT images and voxel-based finite element analysis. Bone 36, 480-8 (2005).

27. Dalzell, N. et al. Bone micro-architecture and determinants of strength in the radius and tibia: age-related changes in a populationbased study of normal adults measured with high-resolution pQCT. Osteoporosis International 20, 1683-94 (2009).

28. Sorensen, A.A., Howard, D., Tan, W.H., Ketchersid, J. \& Calfee, R.P. Minimal clinically important differences of 3 patient-rated outcomes instruments. J Hand Surg Am 38, 641-9 (2013).

29. Geusens, P.P.M.M. \& Boonen, S. Osteoporosis and the Growth Hormone-Insulin-Like Growth Factor Axis. Hormone Research 58, 49-55 (2002).

30. Boonen, S. et al. Musculoskeletal effects of the recombinant human IGF-I/IGF binding protein-3 complex in osteoporotic patients with proximal femoral fracture: a doubleblind, placebo-controlled pilot study. J Clin Endocrinol Metab 87, 1593-9 (2002).
31. Veitch, S.W. et al. Changes in bone mass and bone turnover following tibial shaft fracture. Osteoporos Int 17, 364-72 (2006).

32. Stoffel, K., Engler, H., Kuster, M. \& Riesen, W. Changes in biochemical markers after lower limb fractures. Clin Chem 53, 131-4 (2007).

33. Joerring, S., Krogsgaard, M., Wilbek, H. \& Jensen, L.T. Collagen turnover after tibial fractures. Arch Orthop Trauma Surg 113, 3346 (1994).

34. Cox, G., Einhorn, T.A., Tzioupis, C. \& Giannoudis, P.V. Bone-turnover markers in fracture healing. J Bone Joint Surg Br 92, 32934 (2010).

35. Marsell, R. \& Einhorn, T.A. The biology of fracture healing. Injury 42, 551-5 (2011).

36. Geusens, P., Emans, P.J., de Jong, J.J. \& van den Bergh, J. NSAIDs and fracture healing. Curr Opin Rheumatol 25, 524-31 (2013).

37. Goldhahn, J., Beaton, D., Ladd, A., Macdermid, J. \& Hoang-Kim, A. Recommendation for measuring clinical outcome in distal radius fractures: a core set of domains for standardized reporting in clinical practice and research. Arch Orthop Trauma Surg (2013).

38. Ng, C.Y. \& McQueen, M.M. What are the radiological predictors of functional outcome following fractures of the distal radius? J Bone Joint Surg Br 93, 145-50 (2011). 



$$
x
$$




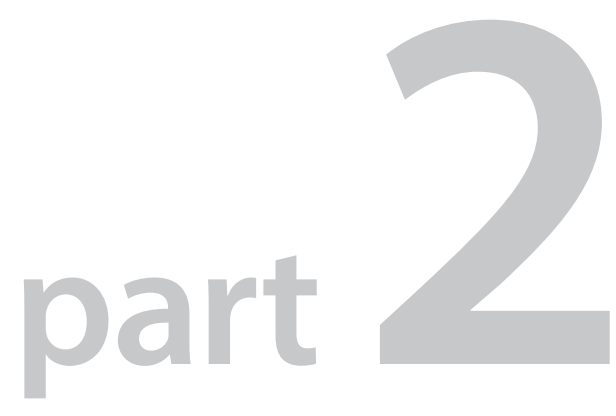

Follow-up two years after a stable distal radius fracture using $\mathrm{HRpQCT}$ 


$$
\frac{b}{x}
$$




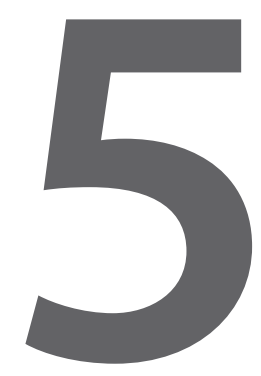

Fracture repair in the distal radius in post-menopausal women: a follow-up two years post-fracture using $\mathrm{HRpQCT}$ 


\section{Abstract}

\section{Background}

Fracture healing is characterized by an intense increase in modeling and remodeling of bone, which allows removal of the cast after a stable distal radius fracture within three to five weeks. However, at that time, bone strength has not recovered yet. We studied the changes in bone mineral density (BMD), micro-architecture and bone stiffness after a distal radius fracture during a two-year follow-up in comparison to the contra-lateral side, and the association between the two-year stiffness and baseline BMD, microarchitecture, and early changes in these parameters.

\section{Methods}

The fractured side of fourteen post-menopausal women (mean age $64 \pm 8$ years) with a conservatively treated distal radius fracture was scanned by high-resolution peripheral quantitative computed tomography (HRpQCT) at one to two, three to four, six to eight, and twelve weeks and two years post-fracture. The same region contra-laterally was scanned as well at the two-year visit. BMD, micro-architecture and stiffness parameters were determined and the fracture side was compared to the contra-lateral side using a linear mixed-effect model. Spearman's correlation was used to correlate the two-year bone stiffness with baseline BMD, micro-architecture, and early three-month changes in these parameters.

\section{Results}

Two years post-fracture, cortical and trabecular thickness and torsional and bending stiffness were significantly higher at the fractured side compared with the nonfractured side $(21 \%, 55 \%, 31 \%$ and $29 \%$, respectively, $p<0.05)$, whereas BMD was similar. Two-year torsional and bending stiffness correlated significantly with baseline BMD and cortical perimeter $(|\mathrm{rho}| \geq 0.63, \mathrm{p}<0.016)$, but not with early changes in bone parameters.

\section{Conclusion}

Using HRpQCT, this study illustrates that fracture healing is not completed by the time the cast is removed. We showed that from six weeks to two years post-fracture large changes occur in BMD, micro-architecture and biomechanical parameters at the fractured side, which were fully recovered after two years in comparison to the nonfractured contra-lateral side. Interestingly, higher two-year torsional and bending stiffness were associated with lower BMD and higher cortical perimeter at baseline. 


\section{Introduction}

Fracture healing is a complex postnatal regenerative process that recapitulates many ontological events of embryonic skeletal development. ${ }^{1}$ The results of intense catabolic and anabolic processes in fracture repair are commonly described by a four-phase model: initiated by inflammation, soft and subsequent hard callus formation consolidates the bone fragments, which is then followed by remodeling of the callus. ${ }^{2} 3$ During the remodeling phase, the structural integrity of the trabecular region and the cortex are remodeled in order to fully restore the bone's biomechanical properties ${ }^{3}$. Whereas the first three phases generally are completed within four to five weeks after fracture ${ }^{1}$, the remodeling phase continues for months to years. ${ }^{3}$

In clinical practice, the recommended follow-up period for conservatively treated distal radius fractures is three weeks for non-displaced fractures and four to five weeks for displaced fractures. ${ }^{4}$ At that time, immobilization of the wrist is no longer continued by removal of the cast and, although the remodeling process has only just started by then, the wrist is not routinely subjected to follow-up for further clinical evaluation. The rationale behind this approach is that the fracture is not likely to displace anymore, since the fragments are stabilized by the presence of hard callus. However, the long-term outcome of the healing fracture, in terms of restored micro-architecture and bone strength, is thus not taken into full consideration.

We previously studied the first three months of fracture healing in stable distal radius fractures with high-resolution peripheral quantitative computed tomography (HRpQCT) and found significant changes in bone density, micro-architecture and bone mechanical competence. ${ }^{5}$ Furthermore, it was shown that early changes in trabecular bone density and separation, and torsional stiffness predicted the clinical outcome twelve weeks post-fracture. ${ }^{6}$ Since the follow-up period in this study was limited to twelve weeks post-fracture, it is unknown to what extent the fractured radius will be restored in terms of bone density, micro-architecture and mechanical properties in the long term. We therefore conducted a follow-up at two years post-fracture to study the long-term restoration of these parameters.

The purpose of this study was threefold: Because we expected large changes at the fracture side in the period from cast removal to two years post-fracture, the first goal was to quantify these changes by comparing the two-year bone parameters at the fracture side to the six- and twelve-week bone parameters at the same side. 
Our second goal was to investigate if the bone was fully recovered at two years post-fracture by comparing the bone density, micro-architectural and mechanical parameters at two years post-fracture between the fractured and the nonfractured contra-lateral side. Last, because the main purpose of fracture healing is the restoration of the bone's mechanical properties, which is accomplished by cortical and trabecular remodeling, our third goal was to investigate if the two-year bone mechanical parameters could be predicted from 1) the initial bone density and micro-architectural parameters, and 2) the early changes in these parameters.

\section{Materials and Methods}

HRpQCT scans were scheduled according to protocol at one to two (baseline), three to four weeks, six to eight weeks and twelve weeks post-fracture, and additionally at two years post-fracture. The short-term study protocol (registration no. NTR3821) and its extension were approved by an independent Medical Ethics Committee.

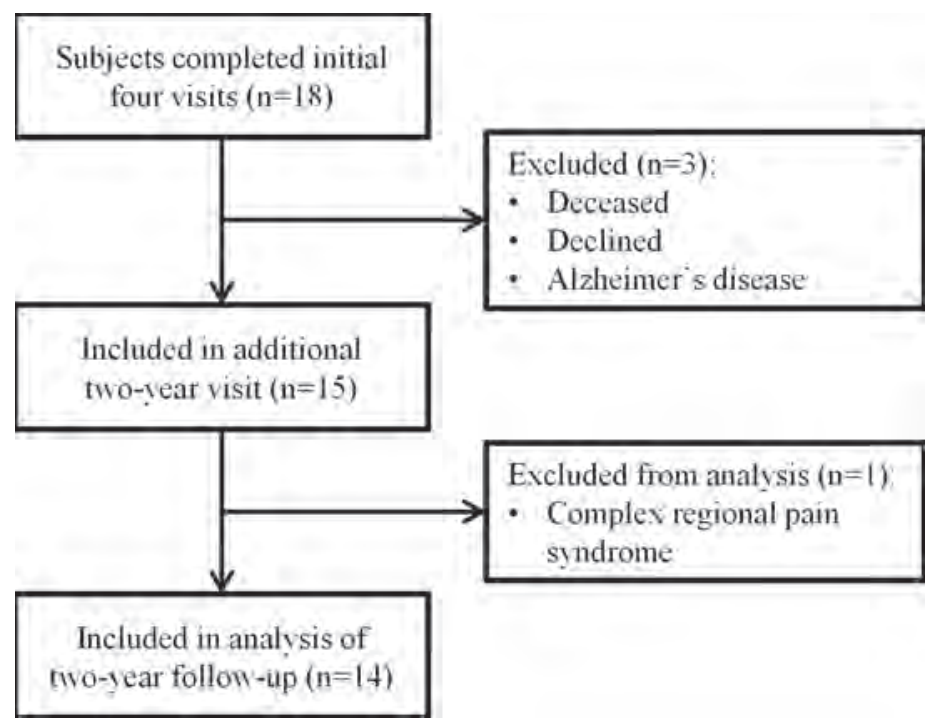

Figure 1 Flow diagram of the number of patients who were included in the two-year follow-up visit.

\section{Subjects}

All eighteen patients who had participated in our previously published study ${ }^{5}$ and were still alive were invited to participate in the additional visit two years postfracture. No additional inclusion and exclusion criteria were applied (Table 1). Of these patients, fourteen were found eligible for inclusion in the analysis of the 
bone parameters at the two-year visit (Figure 1). Patients that agreed to participate in the two-year visit gave written informed consent prior to participation.

\section{Scanning by HRpQCT}

The distal radius at the fractured and the contra-lateral side were scanned by HRpQCT (XtremeCT, Scanco Medical AG, Bruttisellen, Switzerland) using standard clinical settings (peak voltage of $60 \mathrm{kVp}$, tube current of $900 \mu \mathrm{A}$ and $100 \mathrm{~ms}$ integration time). A scout view was made prior to scanning to determine the start of the scan. The region of interest (ROI) at the fractured side was set similarly to the initial four visits ${ }^{5}$, which was based on the initial fracture location and was $18 \mathrm{~mm}$ long. To be able to compare the fracture side to the contra-lateral side, the ROI at the contra-lateral side was chosen the same as the ROI at the fractured side (Figure 2). All images were reconstructed using an isotropic voxelsize of $82 \mathrm{~mm}$, thus each measurement resulted in 220 parallel slices.

To ensure compatibility with the initial visits, the scans at the fracture side were made while patients were wearing a fiberglass cast. The scans at the contralateral side were made without a cast. To prevent motion, the forearms were fixed in a cylindrical carbon holder with an inflatable cushion (Pearltec AG, Schlieren, Switzerland).

After scanning, all HRpQCT images were checked for motion-induced image artifacts and were quality graded according to the manufacturer's guidelines and as described by Pialat et al.? In case of insufficient quality, i.e. grades four and five, the scan was repeated.

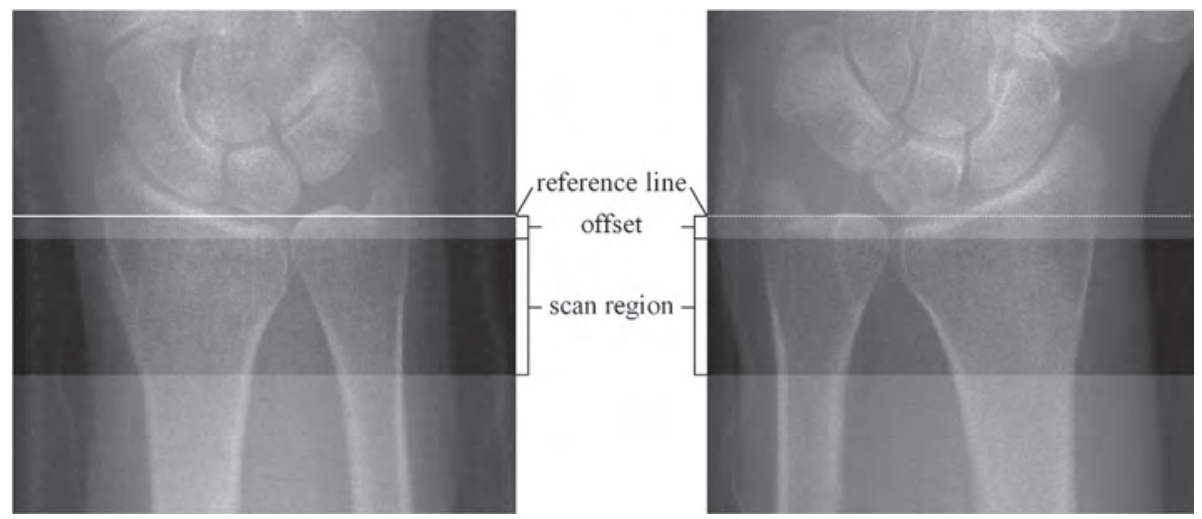

Figure 2 Scout views of the fracture side (left) and contra-lateral side (right) showing the region that was scanned by HRpQCT during the fifth visit at two years post-fracture. At both sides, the proximal edge of the lunate bone was chosen as the landmark for the placement of the reference line. The scan region was $18.04 \mathrm{~mm}$ long, but the offset between the reference line and the start of the scan was different for each patient because it depended on the location of the fracture. 


\section{Evaluation of bone density and micro-architecture}

During all visits, the HRpQCT images of both sides were evaluated using the standard patient evaluation protocol as described earlier by Boutroy et al. ${ }^{8} \mathrm{In}$ short, a semi-automated contouring algorithm was used to derive the periosteal boundary surface of the radius. After contouring, the total region was divided in a trabecular and cortical compartment and the following bone parameters were determined: volumetric bone mineral density $[\mathrm{mgHA} / \mathrm{cm} 3]$ was assessed for the total (Dtot), trabecular (Dtrab) and cortical region (Dcort). ${ }^{9}$ The micro-architectural parameters that were calculated in the trabecular region were the trabecular number (Tb.N) $\left[\mathrm{mm}^{-1}\right]$, thickness (Tb.Th) $[\mathrm{mm}]$ and separation (Tb.Sp) $[\mathrm{mm}]$, which were all determined using a 3D ridge extraction method ${ }^{10}$ and standard morphological relations. For the cortical region, the cortical thickness (Ct.Th) [mm] and the cortical perimeter (Ct.Pm) $[\mathrm{mm}]$ were obtained. ${ }^{9}$

\section{Micro finite element analysis}

Micro finite element ( $\mu \mathrm{FE})$ models were created directly from the segmented HRpQCT images similar to earlier studies. ${ }^{11,12}$ In short, to obtain a representative $\mu \mathrm{FE}$ model of the bone's micro-architecture each voxel in the segmented images that represents bone tissue was converted into a brick element of the same size. These $\mu \mathrm{FE}$ models typically consisted of three to four million elements. A Young's modulus of $10 \mathrm{GPa}$ and a Poisson's ratio of 0.3 were assigned to every element.

Different load cases were applied to simulate clinically relevant loading scenarios, where the associated stiffness was chosen as outcome variable. The first load case represented a 'high friction' compression test with a prescribed displacement in the axial direction of $1 \%$ of the total length, from which the compression stiffness (Scomp) $[\mathrm{kN} / \mathrm{mm}]$ was calculated. The second load case represented a prescribed rotation of $0.01 \mathrm{rad}$ around the longitudinal axis applied to the surface perpendicular to this axis from which the torsional stiffness (Stors) [ $\mathrm{kNmm} / \mathrm{rad}]$ was calculated. A third and fourth load case represented a prescribed rotation of $0.01 \mathrm{rad}$ applied around the sagittal and transversal axes respectively, onto the surfaces perpendicular to each axis, thus inducing a state of pure bending in two directions, from which the bending stiffness in each direction (Ssagittal and Stransversal) was calculated. Because the orientation of the patients' forearm was not exactly the same during all visits, a quadratic mean bending stiffness was calculated (Sbend) $\left[\mathrm{kNmm}^{2}\right]$ from the sagittal and transversal bending stiffness according to Equation 1.

$$
\text { Sbend }=\sqrt{\frac{\text { Ssagittal }^{2}+\text { Stransversal }^{2}}{2}}
$$

(Equation 1) 


\section{Statistical Analysis}

To be able to deal with missing values caused by excluding scans of insufficient quality or missed visits, a linear mixed effectmodel was used. Using this model, the bone density, architectural and mechanical parameters at each visit were expressed as estimated marginal means (EMM) with 95\% confidence intervals. To test if the bone parameters at the fracture side were restored at two years post-fracture, the bone parameters at the fracture side were compared to the bone parameters at the contra-lateral side by pairwise comparison.

To test if the bone parameters at six and twelve weeks post-fracture, i.e. visit three and four, were different at two years-fracture, the bone parameters at visit three and four were compared to the bone parameters at the two-year visit using pairwise comparison. To correct for multiple comparisons, Bonferroni correction was applied.

To correlate the two-year bone biomechanical parameters with the early changes, i.e. from baseline to twelve weeks post-fracture, in bone density and micro-architecture, a summary measure was defined for each parameter as described by Matthews et al. ${ }^{13}$. The following summary measures were tested: slope of the regression line and normalized area under the curve (AUC). Next, these summary measures as well as the baseline value of each bone parameter were correlated to the two-year bone mechanical parameters Scomp, Stors and Sbend using Spearman's correlation coefficient.

A significance level of $\alpha=0.05$ was used for all analyses and all statistical analyses were performed with SPSS Statistics for Windows version 20.0 (IBM Corp., Armonk, NY, USA).

\section{Results}

The mean time between the date of fracture and the two-year visit was 26.5 months (range 22.3-29.3). Of the scans in the seventeen patients in the first four visits and fourteen patients in the two-year follow-up visit, in total nineteen scans (19.8\%) were of insufficient quality due to motion artifacts, even after repetition. These scans were excluded from the statistical analysis of the bone parameters.

\section{Restoration of the fracture side}

Visual inspection of the follow-up HRpQCT images of the healing distal radius fractures showed that all cortical gaps were completely restored at two years post-fracture in all participants (Figure 3). In general, the images suggest that the cortex is denser and thicker at two years post-fracture in comparison to the cortex 
at the earlier visits. Also, the images suggest that remodeling of the trabecular structure has occurred during the two year healing period because fewer trabeculae seem to be present at the two-year image compared to the twelve-week image. Furthermore, the blurring in the trabecular region, which was visible at three up to twelve weeks post-fracture, has disappeared at two years post-fracture.

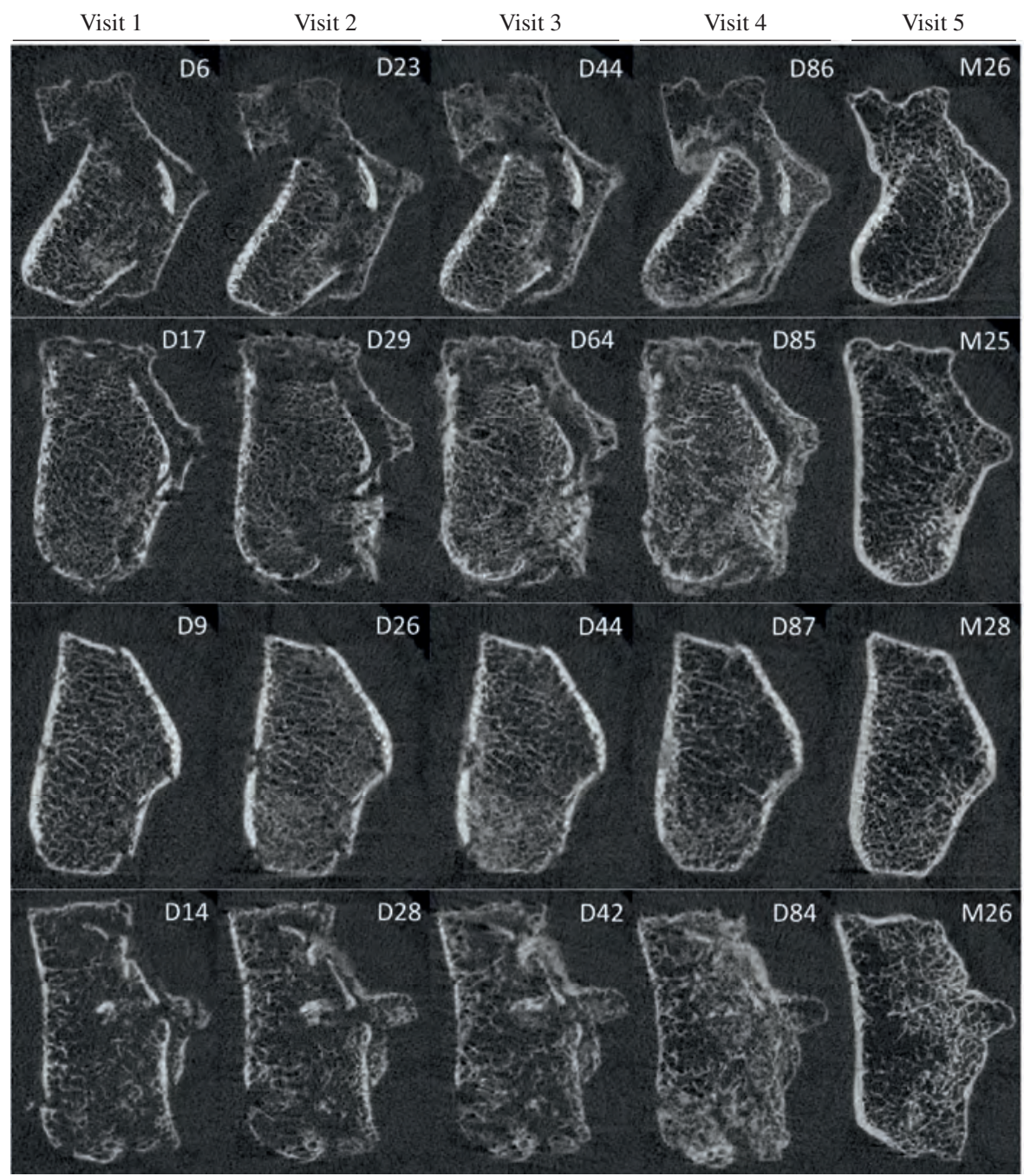

Figure 3 Longitudinal HRpQCT slices of four fractures showing the formation of woven bone (visits two and three) and bridging of the cortex (visit four). At two years post-fracture (visit five), the cortices suggested to be denser and thicker and the trabecular compartment appeared to show signs of deterioration compared with baseline. 
Table 1 Inclusion and exclusion criteria for the initial study

\begin{tabular}{ll}
\hline Inclusion criteria & Exclusion criteria \\
\hline Postmenopausal women & Previous fracture at current location \\
Older than 50 years & Active or suspected infection \\
Stable distal radius fracture & Malignant disease in the past 12 months \\
Treated by cast immobilization & Neuromuscular or neurosensory deficit which would limit the \\
Willing and able to participate & ability to assess performance \\
& Known systemic or metabolic disorders leading to progressive \\
& bone deterioration \\
& Active inflammatory disease \\
& Use of oral glucocorticoids \\
& Mental incompetence \\
& Severe concurrent joint involvements \\
& Selection for other trial on distal radius fractures \\
&
\end{tabular}

The bone density, micro-architectural and biomechanical parameters at the fracture side at each visit, as well as the bone parameters at the contra-lateral side at the fifth visit are presented in Table 2. Time series line plots of the bone parameters at fracture side together with the side-by-side comparison at two years post-fracture are plotted in Figure 4.

From twelve weeks post-fracture to two years post-fracture, only Dtot, Tb.Th and Ct.Pm did not change significantly. The other parameters either increased or decreased significantly in this period. While Dtot did not change significantly, Dtrab decreased further with $21.1 \%(p=0.002)$, and Dcort increased with $13.5 \%$ ( $p$ $<0.001)$. For the micro-architectural parameters, Tb.N decreased with $18.6 \%$ ( $p=$ 0.002 ) and Tb.Sp increased by 33.9\%. Ct.Th increased with $31.4 \%$ ( $p<0.001)$. Also the bone mechanical parameters Scomp, Stors and Sbend increased, with $47.2 \%$, $67.5 \%$ and $96.8 \%$ (all $p<0.001$ ), respectively.

Similar results were found for the change in bone parameters from six weeks post-fracture to two years-post fracture (see Table 1). In general, the changes in this period were larger, except for Dcort, where the increase was $12.7 \%(p<0.001)$.

\section{Comparison to contra-lateral side}

At two years post-fracture trabecular and cortical thickness were significantly higher at the fracture side ( $+55.5 \%, p<0.001$ and $+19.8 \%, p=0.047$, respectively) than at the contra-lateral side. Also, Stors and Sbend were significantly higher at the fracture side than at the contra-lateral side $(+30.7 \%, p=0.016$ and $+29.5 \%, p$ $=0.030$, respectively). Scomp, however, did not differ significantly between both sides. The other parameters, i.e. bone density parameters Dtot, Dtrab and Dcort and micro-architectural parameters Tb.N and Tb.Sp, and Ct.Pm, were also not significantly different between the fracture and the contra-lateral side at two years post-fracture. 


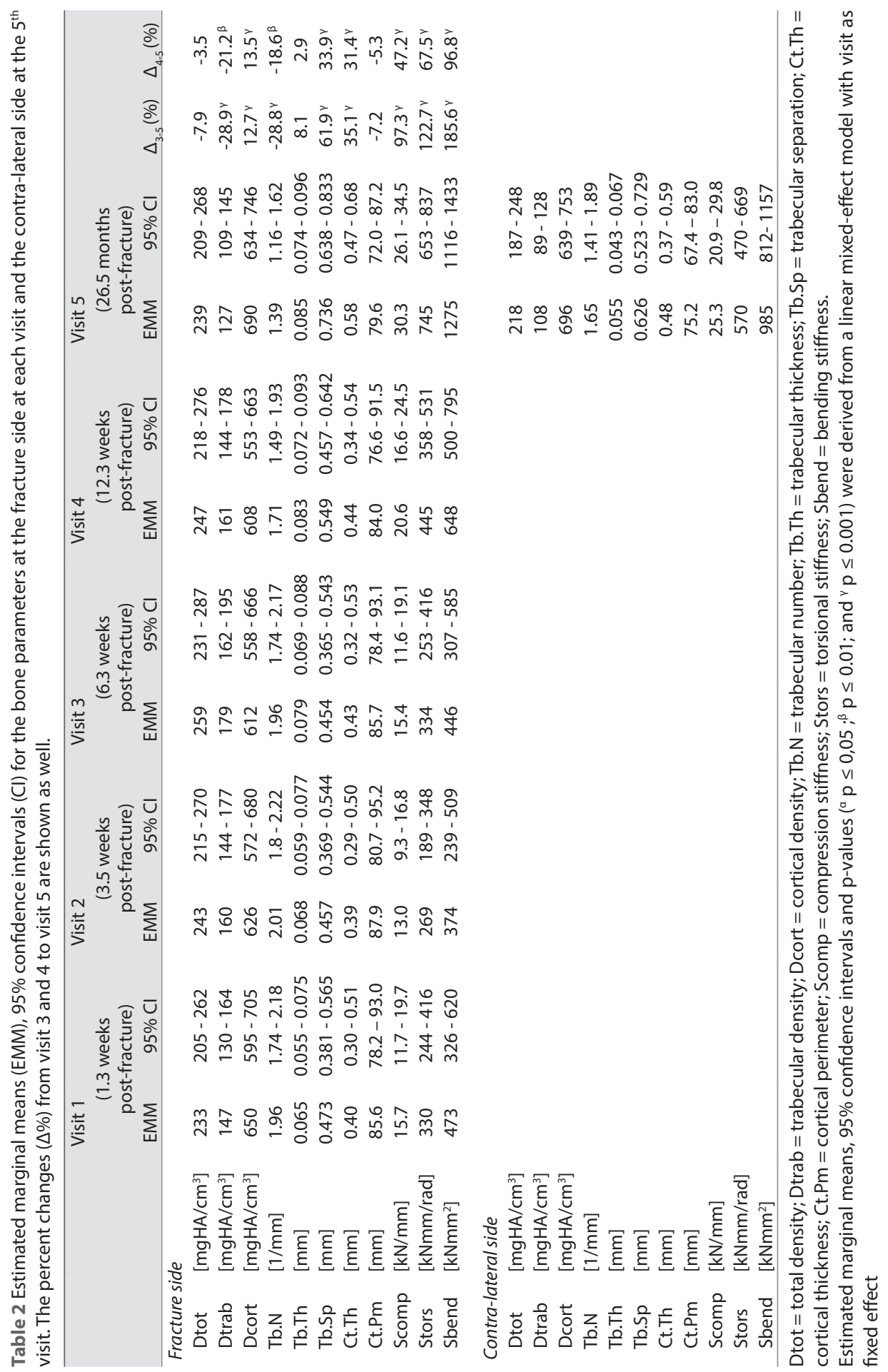




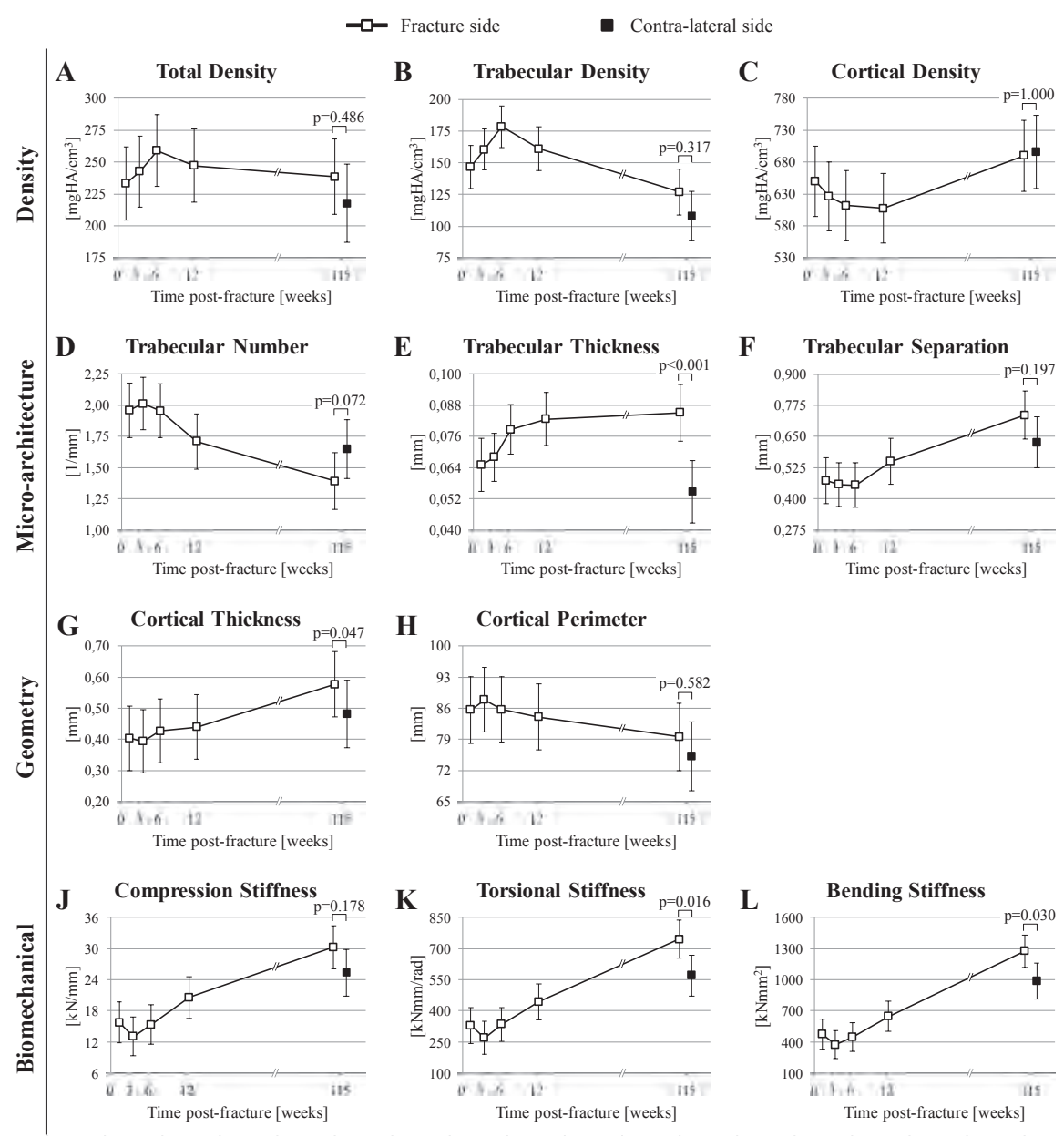

Figure 4 Time series line plots of the HRpQCT-derived bone density $(A-C)$, micro-architectural (D-F), geometrical $(\mathrm{G}, \mathrm{H})$, and biomechanical $(\mathrm{J}-\mathrm{L})$ parameters at the fracture side (white squares) and the twoyear post-fracture parameters at the contra-lateral side (black squares). Estimated marginal means and $95 \%$ confidence intervals are calculated using a linear mixed-effect model. The $p$-values for the pairwise comparison between the fracture and contra-lateral side at the two-year post-fracture visit are obtained from the same linear mixed-effect model.

\section{Relation between two-year bone stiffness and baseline/early changes in BMD} and micro-architecture

The two-year compression stiffness Scomp correlated significantly with the early change in Dcort, expressed by the slope (rho $=-0.670, p=0.009$ ) and the normalized AUC ( $r h o=-0.749, p=0.002$ ). The two-year Scomp did not correlate to any of the bone parameters at baseline.

Two-year torsional stiffness correlated significantly with Dtot (rho $=-0.666, p$ $=0.009$ ) and Ct.Pm ( $r h o=0.666, p=0.009$ ) at baseline (Figure 5), but not with the 
early changes in any of the bone parameters. Similarly, two-year bending stiffness correlated significantly with Dtot $(r h o=-0.631, p=0.016)$ and Ct.Pm (rho $=0.653, p$ $=0.011$ ) at baseline, but not with the early changes in any of the bone parameters.

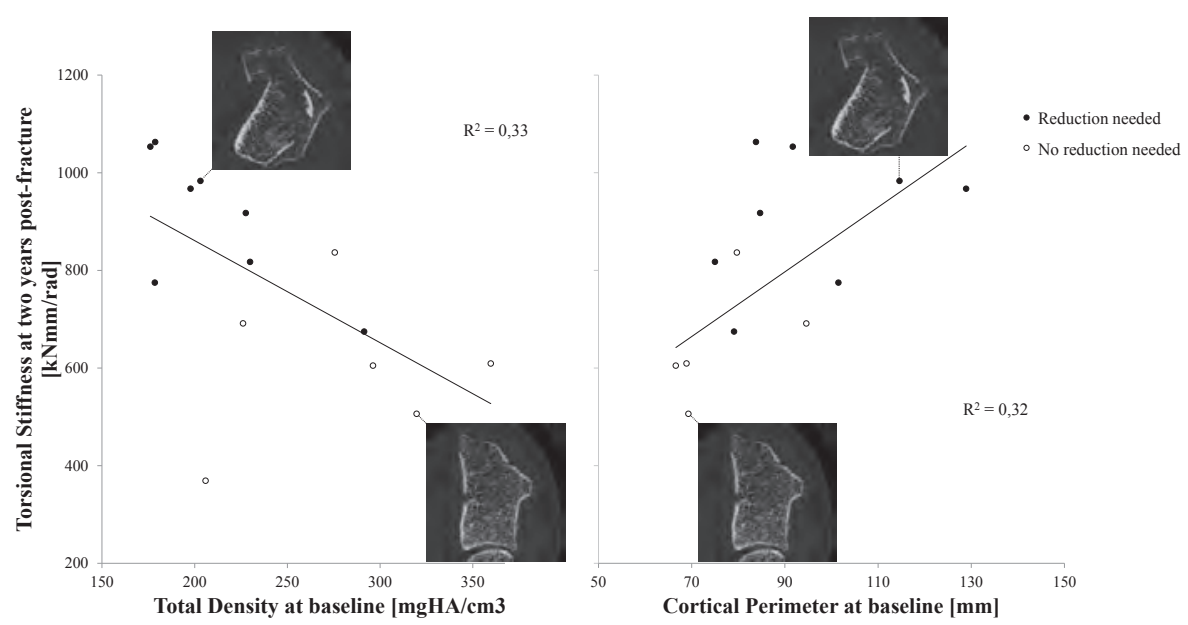

Figure 5 Correlations of the total density Dtot (right) and cortical perimeter Ct.Pm (left) at baseline and the two-year bone torsional stiffness. Representative transversal slices of two fractures ( $\bullet=$ reduced; o = non-reduced) are overlaid and indicate that the reduced fractures with a generally lower Dtot and higher Ct.Pm at baseline result in a higher two-year torsional stiffness.

\section{Discussion}

To our knowledge, this study represents the first use of non-invasive high-resolution in vivo imaging to monitor the healing process of metaphyseal fractures with a follow-up period of two years. Compared to the contra-lateral side at two years post-fracture, the fractured side had thicker cortices and trabeculae while BMD was similar. Ultimately, this resulted in a restoration of bone stiffness that ended up being significantly higher at the fracture side than at the contra-lateral side.

\section{Changes at the fracture side}

Changes in the bone parameters during the first three months of fracture healing were published earlier ${ }^{5}$, but there a comparison with baseline was made. The additional visit at two years post-fracture in the current study allowed us to examine to which extent the bone parameters change after the cast has been removed. Interesting were the remarkable changes that we found in almost all bone density, micro-architectural and biomechanical parameters. Both the HRpQCT images as 
well as the evaluation of the bone parameters revealed that in the period from six weeks to two years post-fracture, the cortex became thicker while concurrently there was a decrease in trabecular number and increase in trabecular separation. These changes in the trabecular region might be caused by the thickening of the cortex: as with a thicker cortex the load-bearing shifts towards the cortical region, and less load is carried by the trabecular region which ultimately could lead to resorption of the trabecular bone.

Simultaneous to the cortical and trabecular changes, bone stiffness showed a major increase of more than $100 \%$. This result thus suggests that by the time the cast is removed, the bone mechanical properties as measured by HRpQCT and $\mu$ FEA are far from recovered. From Figure 3, however, it is apparent that more complex fractures do not remodel back to the situation before fracture, whereas the less displaced fractures appear to do so. Additional statistical analysis with stratification by group (reduction versus no reduction required) revealed that for the fractures that did not need reduction the stiffness parameters at the fracture side at any visit did not significantly differ from the contra-lateral side at two years post-fracture. For the reduced fractures, on the other hand, the stiffness parameters at the first three visits were all significantly lower and at the last visit significantly higher at fracture side in comparison to the contra-lateral side at the last visit (data not shown). Despite the low number of subjects in each group, this might indicate that less complex fractures are faster recovered biomechanically speaking than more complex fractures.

Clinically speaking, on the other hand, all patients in this study were considered sufficiently recovered at the time the cast was removed, regardless of whether reduction was required during treatment. The difference between being recovered clinically and biomechanically indicates that a $100 \%$ recovery of bone strength is not required for normal daily activities. Indeed, clinicians primarily advise their patients to practice on regaining normal flexion and extension, and to load their arm gradually as pain allows. Loading the fractured wrist up to the same amount as before fracture is thus not recommended at that time, advice that is now supported by the results we presented here.

\section{Comparison to contra-lateral side}

As can be seen in Figures $4 \mathrm{~J}-\mathrm{L}$, the bone compression, torsional and bending stiffness are all at least restored at two years post-fracture. However, the torsional and bending stiffness exceeded the contra-lateral values significantly, whereas this was not the case for stiffness in compression. Although this difference might be caused by a lack of power owing to the low number of subjects, it is also possible that there is a true difference between the recovery of the different stiffnesses. 
From a clinical perspective, bone compressive and bending strength are most important as they determine whether the bone is strong enough to withstand the bending and compressive forces another fall on the outstretched arm, while torsional stiffness seem to be of less importance in this case.

\section{Correlation early changes in bone parameters and bone stiffness at two years post-fracture}

An interesting finding of this study is that a higher bone compression stiffness at two years post-fracture is associated with a higher early decrease in cortical density. A likely explanation is that newly formed cortical bone is incorporated into the cortical mask as soon as it reaches a density that is above the used threshold. Because this new bone is still not as dense as the old cortical bone, including it into the cortical mask leads to a decrease in the overall cortical density. Our results suggest that the sooner this happens and the cortical gaps are filled with new bone, the better the compression stiffness will be at two years post-fracture.

Opposite to two-year compression stiffness, we found no association between the two-year torsional and bending stiffness and the early changes in bone density or micro-architecture. Instead, two-year torsional and bending stiffness were associated with baseline values of BMD and cortical perimeter. Interestingly, this relation seems to be linked to whether a fracture required reduction (Figure 5). An explanation for this might be that a nonreduced fracture most likely was not displaced and thus would not have a distal fragment overlapping its proximal part and therefore will have a normal perimeter. Displaced fractures that were reduced, however, will have an increased perimeter due to the overlap of a distal segment over its proximal part. Because the outer perimeter is used as the boundary of the ROI for the evaluation of the bone parameters, the total density is affected as well. Although the displaced fractures were reduced to within normal limits during treatment, some overlap might be unavoidable. During healing, these overlapping fragments grow back together and thus lead to a large amount of bone mass on the outer side of the bone. This finding is consistent with the standard rules of mechanics that tell us that adding material to the outer side of a volume has a positive influence on the volume's torsional and bending stiffness and less to no effect on the compression stiffness.

\section{Limitations}

An important limitation of our study is that at six and twelve weeks post-fracture, cartilaginous or low-mineralized tissue is present at the fracture side to give support and stability. Since these tissues are not taken into account in the $\mu$ FEA, the true stiffness at the fractured side at these time points might be underestimated 
by $\mu$ FEA. For a more accurate estimation of the bone stiffness at these time points, these tissues should be included into the $\mu \mathrm{FE}$ model as well, for example by combining the HRpQCT data with information from quantitative magnetic resonance imaging. Another approach that allows in vivo assessment of bone strength in healing fractures is radiostereometric analysis (RSA). ${ }^{14,15}$ Although a prescribed load has to be applied onto the fracture and markers need to be placed onto the bone fragments, which can be problematic in particularly the first weeks post-fracture, RSA could be a potential next step in future studies on bone strength in healing wrist fractures.

Another limitation is that the number of subjects is small and some measurements were of insufficient quality, leading to a low number of usable measurements. However, despite the exploratory nature of the study and thanks to the use of an advanced statistical model that is able to deal with these missing values, we can still make some strong conclusions.

Also, in the side-to-side comparison we assumed that there is no bilateral asymmetry in bone parameters at the distal radius. This assumption is partially legitimate, as a recent HRpQCT study of Hildebrandt et al. found no left-right differences in the BMD and micro-architectural parameters at the distal radius, whereas the bone failure load was found to be slightly higher at the dominant side (+3.0\%). ${ }^{16}$ However, because most fractures in our study occurred at the nondominant side ( $n=9,74 \%)$, correcting for fracture side would probably strengthen the conclusions.

Another limitation is that, unfortunately, we did not scan the fracture at other time points, e.g. six and twelve months. We do not know whether full recovery already was obtained earlier than two years post-fracture.

Last, we performed a large set of correlations without correcting for multiple testing. The results regarding the correlation between the early changes in bone density and micro-architecture and the two-year bone stiffness should therefore be interpreted with caution, and should mainly be used as an indication for further studies on HRpQCT and fracture healing.

In conclusion, based on HRpQCT measurements in women with a stable distal radius fracture, this study shows that the fractured radius at two years post-fracture has a thicker cortex and thicker trabeculae in comparison with the contra-lateral side. Furthermore, bone torsional and bending stiffness at two years post-fracture are significantly higher than at the non-fractured contra-lateral side. Interestingly, higher torsional and bending stiffness at two-year post-fracture are associated with lower BMD and higher cortical perimeter at baseline. 


\section{Disclosures}

BvR is a consultant for Scanco Medical AG. JJCA is a board member of workgroup Biotechnology of the Dutch Orthopedic Association (NOV). PCW is a board member of the Dutch Spine Society (association of spine surgeons). All other authors state that they have no conflicts of interest.

\section{Acknowledgements}

The study (Dutch trial register NTR3821) was supported by The Weijerhorst Foundation (grant no. WH2).

Authors' roles: Study design: JdJ, JJCA, PCW, BvR, PPG and JvdB. Data acquisition and analysis: JdJ. Data interpretation: All authors. Drafting or critically revising the manuscript: All authors. Approving final version of the manuscript: All authors. JdJ takes responsibility for the integrity of the data analysis. 


\section{References}

1. Einhorn, T.A. \& Gerstenfeld, L.C. Fracture healing: mechanisms and interventions. Nat Rev Rheumatol 11, 45-54 (2015).

2. Pivonka, P. \& Dunstan, C.R. Role of mathematical modeling in bone fracture healing. Bonekey Rep 1, 221 (2012).

3. Marsell, R. \& Einhorn, T.A. The biology of fracture healing. Injury 42, 551-5 (2011).

4. The Association of Surgeons of the Netherlands. Guideline Distal Radius Fractures: Diagnosis and Treatment (Dutch) (Utrecht, 2010).

5. de Jong, J.J. et al. Assessment of the healing process in distal radius fractures by highresolution peripheral quantitative computed tomography. Bone 64C, 65-74 (2014).

6. Meyer, U. et al. Early changes in bone density, micro-architecture, bone resorption, and inflammation predict the clinical outcome 12 weeks after conservatively treated distal radius fractures: an exploratory study. J Bone Miner Res 29, 2065-73 (2014).

7. Pialat, J.B., Burghardt, A.J., Sode, M., Link, T.M. \& Majumdar, S. Visual grading of motion induced image degradation in high-resolution peripheral computed tomography: impact of image quality on measures of bone density and microarchitecture. Bone 50, 111-8 (2012).

8. Boutroy, S., Bouxsein, M.L., Munoz, F. \& Delmas, P.D. In vivo assessment of trabecular bone micro-architecture by high-resolution peripheral quantitative computed tomography. J Clin Endocrinol Metab 90, 6508-15 (2005).

9. Laib, A., Hauselmann, H.J. \& Ruegsegger, P. In vivo high-resolution 3D-QCT of the human forearm. Technology and health care : official journal of the European Society for Engineering and Medicine 6, 329-37 (1998).
10. Hilderbrand, T., Laib, A., Müller, R., Dequeker, J. \& Rüegsegger, P. Direct Three-Dimensional Morhpometric Analysis of Human Cancellous Bone: Microstructural Data from Spine, Femur, Iliac Crest, and Calcaneus. Journal of Bone an Mineral Research 17, 1167 1174 (1999).

11. Pistoia, W. et al. Estimation of distal radius failure load with micro-finite element analysis models based on three-dimensional peripheral quantitative computed tomography images. Bone 30, 842-8 (2002).

12. Dalzell, N. et al. Bone micro-architecture and determinants of strength in the radius and tibia: age-related changes in a populationbased study of normal adults measured with high-resolution pQCT. Osteoporos Int 20, 1683-94 (2009).

13. Matthews, J.N., Altman, D.G., Campbell, M.J. \& Royston, P. Analysis of serial measurements in medical research. BMJ 300, 230-5 (1990).

14. Solomon, L.B. et al. The accuracy and precision of radiostereometric analysis in monitoring tibial plateau fractures. Acta Orthop 81, 487-94 (2010).

15. Madanat, R., Makinen, T.J., Moritz, N., Mattila, K.T. \& Aro, H.T. Accuracy and precision of radiostereometric analysis in the measurement of three-dimensional micromotion in a fracture model of the distal radius. J Orthop Res 23, 481-8 (2005).

16. Hildebrandt, E.M., Manske, S.L., Hanley, D.A. \& Boyd, S.K. Bilateral Asymmetry of Radius and Tibia Bone Macroarchitecture and Microarchitecture: A High-Resolution Peripheral Quantitative Computed Tomography Study. J Clin Densitom (2015). 


$$
\frac{b}{x}
$$




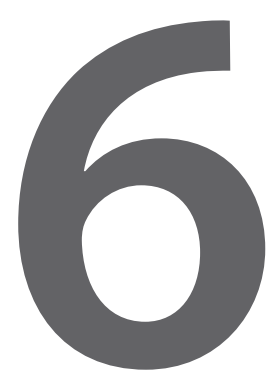

Contra-lateral bone loss at the distal radius in postmenopausal women after a distal radius fracture: a two-year follow-up HRpQCT Study 


\section{Abstract}

\section{Introduction}

Opposite to the fracture side, bone mineral density (BMD) measured by DXA at the contra-lateral side does not change after a distal radius fracture. However, it is unknown if also bone micro-architecture and strength at the contra-lateral side are unaffected. Therefore, the aim of this study was to assess BMD, micro-architecture and bone mechanical properties at the contra-lateral side during two years follow-up after a distal radius fracture using high-resolution peripheral quantitative computed tomography (HRpQCT).

\section{Methods}

The contra-lateral distal radius of fourteen postmenopausal women (mean age 64 \pm 8 years) with a distal radius fracture treated by cast immobilization was scanned by HRpQCT at baseline, three months and two years post-fracture. BMD and cortical and trabecular micro-architecture were measured and biomechanical parameters were estimated using micro finite element analysis. Additionally, markers of bone resorption and formation were measured at each visit. Bone parameters and turnover markers across the three visits were analyzed using a linear mixed-effect model with Bonferroni correction.

\section{Results}

Two years post-fracture, a significant decrease from baseline was found in cortical BMD $(-3.9 \%, p<0.001)$, failure load $(-5.6 \%, p=0.007)$, stiffness in compression $(-5.1 \%, p$ $=0.014)$ and bending $(-6.0 \%, p=0.029)$, and bone formation $(-50.9 \%, p=0.010)$. No significant changes from baseline were observed in total and trabecular BMD, nor in cortical or trabecular micro-architecture and neither in bone resorption. Results were similar between patients with or without adequate anti-osteoporosis drug treatment.

\section{Conclusion}

We found a significant decline in BMD in the cortical but not the trabecular region, and a reduction in bone strength and stiffness at the contra-lateral side two years after a distal radius fracture. These changes exceeded the changes that may be expected due to aging, even in the presence of adequate anti-osteoporosis treatment. 


\section{Introduction}

After fracture of a lower extremity, bone loss often occurs at the fracture side, even at locations more distant from the fracture, due to a period of consequent absence of weight-bearing and immobilization. ${ }^{1,2}$ Bone loss has also been observed at the contra-lateral side after a hip fracture up to two years post-fracture ${ }^{3-6}$ (but not after a tibial shaft fracture ${ }^{2}$.

Following Wolff's law, which states that a bone's internal structure and shape adapt itself to the mechanical loading conditions imposed on it ${ }^{7}$, it is not surprising that the bone at the contra-lateral hip is resorbed after a period of decreased loading due to bed-rest or immobilization. This effect is therefore not expected at non-weight-bearing limbs, such as the forearm, for which the contra-lateral side is not immobilized. Indeed, several studies reported no changes in BMD at the contra-lateral radius one year after a Colles' fracture. ${ }^{8,9}$

Whereas the aforementioned studies only took areal bone mineral density (aBMD) assessed by dual-energy $\mathrm{x}$-ray absorptiometry (DXA) into account, we recently used high-resolution peripheral quantitative computed tomography in combination with micro finite element analysis ( $\mu \mathrm{FEA}$ ) to assess changes in volumetric bone mineral density (vBMD), bone micro-architecture and bone strength during the healing process of distal radius fractures. ${ }^{10,11}$ In this study, large changes in $\mathrm{VBMD}$, micro-architecture and biomechanical parameters were found at the fracture side during a follow-up of two years post-fracture. Also, we found no changes in these bone parameters at the contra-lateral side during the first three months post-fracture ${ }^{10}$, but it is unknown if the bone parameters remain unchanged during a longer time post-fracture.

Since earlier studies found no changes in aBMD at the contra-lateral side after a forearm fracture ${ }^{8,9}$, we hypothesized that after the initial three-month period of no change, no changes in VBMD and therefore also not in micro-architecture and bone strength occurs at the contra-lateral side during the two years post-fracture. To address these hypotheses, we monitored VBMD, micro-architecture and bone biomechanical properties at the contra-lateral radius using HRpQCT and $\mu \mathrm{FEA}$ during a period of two years after a distal radius fracture. 


\section{Methods}

\section{Subjects}

The patients in this study all participated in a medical ethical approved clinical trial on fracture healing (registration no. NTR3821). ${ }^{11}$ Besides the initial inclusion and exclusion criteria (Table 1), an additional exclusion criterion for the current study was a previous fracture at the contra-lateral side. All patients were informed and gave written informed consent prior to participation.

\section{HRpQCT scanning}

HRpQCT (XtremeCT, Scanco, Brüttisielen Switzerland) scans of the contra-lateral forearm were performed at baseline (one to two weeks post-fracture) and three months and two years post-fracture. The scans were made at standard clinical highresolution settings, i.e. with a tube voltage of $60 \mathrm{kVp}$, tube current of $900 \mu \mathrm{A}$ and a $100 \mathrm{~ms}$ integration time. All scans were reconstructed using an isotropic voxelsize of $82 \mathrm{~mm}$. At baseline and the first follow-up visit, the region of interest (ROI) incorporated a $9 \mathrm{~mm}$ long section starting $9.5 \mathrm{~mm}$ proximal of the os lunate bone in all patients. Due to changes in the study protocol, the ROI at the two-year followup visit was extended to a $18 \mathrm{~mm}$ long section. The start of this section differed between patients such that it matched the scanned region at fracture side."

To prevent motion artifacts, the forearm of each patient was fixed in a cylindrical carbon holder (XCT Carpal, Pearltec AG, Switzerland) with an inflatable cushion as described previously. ${ }^{12}$ After scanning, all HRpQCT images were checked for motion-induced image artifacts and were quality graded according to the manufacturer's guidelines and as described by Pialat et al. ${ }^{13}$ In case of insufficient quality, i.e. grade four and five, the scan was repeated once.

Quality control during the study period was assured by regular HRpQCT measurements of a calibration phantom containing rods of different densities $(0$, $100,200,400$, and $800 \mathrm{mgHA} / \mathrm{cm}^{3}$ ).

\section{Evaluation of bone density and micro-architecture}

Since the scanned region at the last visit differed from the first two visits, the common ROI was determined first by matching the first and last slices of the follow-up scans to the baseline scan. The bone density and micro-architectural parameters within this common $\mathrm{ROI}$ were then evaluated using the standard patient evaluation protocol provided by the manufacturer (Scanco Medical AG) as described earlier. ${ }^{14}$ In short, after semi-automatically deriving the periosteal contour the total region was divided in a cortical and trabecular region and each region was segmented and combined to into a 3D model to represent the micro- 
architecture. vBMD $\left[\mathrm{mgHA} / \mathrm{cm}^{3}\right]$ was calculated in the total (Dtot), trabecular (Dtrab) and cortical (Dcort) region. Furthermore, trabecular number (Tb.N) [ $\left.\mathrm{mm}^{-1}\right]$, thickness (Tb.Th) [mm] and separation (Tb.Sp) [mm] were determined to assess the trabecular micro-architecture, and cortical thickness (Ct.Th) [mm] and perimeter (Ct.Pm) [mm] were assessed for the cortical compartment. In addition to the standard patient evaluation protocol, extended cortical analysis as described by Burghardt et al. ${ }^{15}$ was performed to assess cortical porosity (Ct.Po) [\%], cortical pore volume (Ct.Po.V) $\left[\mathrm{mm}^{3}\right]$ and diameter (Ct.Po.V) [mm].

\section{Finite element analysis}

To assess the bone's mechanical properties, micro finite element ( $\mu \mathrm{FE}$ ) models were created directly from the segmented HRpQCT images similar to earlier studies. ${ }^{16,17}$ In short, to obtain a representative $\mu \mathrm{FE}$ model of the bone's micro-architecture each voxel in the segmented images that represents bone tissue was converted into a brick element of the same size. These $\mu \mathrm{FE}$ models typically consisted of around 1 million elements. Constant material properties were assigned to each element, i.e. a Young's modulus of $10 \mathrm{GPa}$ and a Poisson's ratio of 0.3

Next, a compression load was simulated by applying a 'high friction' compression test with a prescribed displacement in the axial direction of $1 \%$ of the total length, from which the compression stiffness (Scomp) $[\mathrm{kN} / \mathrm{mm}$ ] and ultimate failure load (F.Ult) [kN] were estimated.

Additionally, by applying representative loading scenarios also torsional (Stors) $[\mathrm{kNmm} / \mathrm{rad}]$ and bending stiffness (Sbend) $\left[\mathrm{kNmm}^{2}\right]$ were estimated in a similar way as in our previous study and which has been described earlier. ${ }^{10}$

\section{DXA and biochemical analyses}

In accordance with Dutch standard care when a patient over the age of 50 years comes to clinic with a fracture, all patients in this study visited the fracture liaison service (FLS) between four and eight weeks post-fracture for assessment of osteoporosis and vertebral fractures by DXA(Hologic Discovery A; Hologic Inc. Waltham, MA). If the patients already had had a DXA scan in the last year prior to the fracture, this previous DXA measurement was used.

Additionally, blood serum samples were taken for biochemical analyses that included calcium (Photometric assay; Roche Diagnostics, Basel, Switzerland), 25-hydroxyvitamin D (chemiluminescence immunoassay; IDSiSYS Immunodiagnostic Systems GmbH, Frankfurt, Germany), parathyroid hormone (chemiluminescence immunoassay; Siemens, Erlangen, Germany), thyroid-stimulating hormone (electro-chemiluminescence immunoassay; Roche Diagnostics, Basel, Switzerland) and free thyroxine (fluorescence immunoassay; 
Perkin Elmer, Turku, Finland) for diagnosis of hyperthyroidism, hypercalcemia and hyperparathyroidism, which are known contributors to bone loss. ${ }^{18}$ Furthermore, patients were asked about their current medication. If patients were diagnosed with osteoporosis, adequate anti-osteoporosis treatment was started by bisphosphonates, denosumab, calcium or vitamin D, or a combination of these medications. At the end of the study, a telephone interview was conducted with each patient to check whether the patients were compliant to their treatment.

\section{Serum markers of bone turnover}

In addition to the standard biochemical analyses, venous blood samples were collected at each visit by puncture of the antecubital vein for assessment of bone turnover markers. Serum was separated from the bloodclot within 20 minutes and freezed to $-20{ }^{\circ} \mathrm{C}$ until analysis. Serum bone markers representing bone resorption (carboxy-terminal telopeptide of type I collagen (ICTP)) and bone formation (procollagen type-I N-terminal propeptide (PINP)) were assessed by radioimmunoassay (UniQ ${ }^{\circledR}$ PINP/ICTP; Orion Diagnostics, Espoo, Finland) in a certified laboratory. The calculated precision profile indicates a coefficient of variation below $10 \%$ over the range of 2.5 to $25 \mathrm{mg} / \mathrm{L}$ for ICTP and over the range of 25 to $140 \mathrm{mg} / \mathrm{L}$ for PINP. The observed lower limit of blank was $0.6 \mathrm{mg} / \mathrm{L}$ for ICTP and $2 \mathrm{mg} / \mathrm{L}$ for PINP.

\section{Statistics}

Patient's demographic and clinical baseline characteristics were reported in means and their standard deviations. HRpQCT derived bone parameters and the bone turnover markers at each visit were reported as estimated marginal means and their 95\% confidence intervals which were obtained from a linear mixed effect model with visit as fixed effect and a compound symmetry covariance structure. The same linear mixed-effect model with Bonferroni correction was used to compare the bone parameters and bone turnover markers between the three visits.

A level of significance of 0.05 was used in all statistical analyses, and all analyses were performed with SPSS Statistics for Windows version 20.0 (IBM Corp., Armonk, USA).

\section{Results}

From the fifteen patients who completed all visits, one patient developed a complex regional pain syndrome. Fourteen patients were therefore found to be eligible and were included in the analyses. The demographic and clinical baseline 
characteristics of these patients are presented in Table 2. At baseline, seven patients were already receiving calcium and/or vitamin D supplementation, and three of them were also being treated with alendronate. At three months post-fracture, anti-osteoporosis treatment was started (or continued) at the FLS: calcium and/or vitamin D supplementation started (or continued) in all patients. Additionally, seven patients started and three patients continued with alendronate $70 \mathrm{mg}$ per week, and one patient started with subcutaneous injections of $60 \mathrm{mg}$ denosumab every six months. The end-of-study telephone interview with each patient confirmed that the denosumab injections were administered correctly, and that compliance with alendronate treatment was $67 \%$. One patient could not be contacted.

From the in total $42 \mathrm{HRpQCT}$ measurements, nine measurements (21.4\%) were of insufficient quality, due to motion artifacts. The average common ROI between the HRpQCT scans at the three visits consisted of 100.7 slices (91.5\%).

Table 1 Inclusion and exclusion criteria

\begin{tabular}{ll}
\hline Inclusion criteria & Exclusion criteria \\
\hline Postmenopausal women & Previous fracture at the same or contra-lateral location \\
Older than 50 years & Active or suspected infection \\
Stable distal radius fracture & Malignant disease in the past 12 months \\
Treated by cast immobilization & Neuromuscular or neurosensory deficit which would limit the \\
Willing and able to participate & ability to assess performance \\
& Known systemic or metabolic disorders leading to progressive \\
& bone deterioration \\
& Active inflammatory disease \\
& Use of oral glucocorticoids \\
& Mental incompetence \\
& Severe concurrent joint involvements \\
& Selection for other trial on distal radius fractures \\
&
\end{tabular}

\section{Changes in bone density}

In Table 3, the HRpQCT derived bone parameters as well as the percentage change from baseline at each visit within the common ROI are presented. During two years post-fracture, no significant changes occurred in total or trabecular vBMD. For cortical vBMD an initial twelve-week period without change was followed by a decline that led to a change from baseline of $-3.9 \%(p<0.001)$ at two years post-fracture. Separate analysis of the two-year changes in cortical vBMD in the patients who were compliant with anti-resorptive treatment, i.e. alendronate or denosumab, that started three months post-fracture $(n=5)$ and the patients who did not receive or were not compliant with anti-resorptive treatment $(n=6)$, showed similar decreasing trends in cortical BMD (Figure 1, left panel). 
Table 2 Patients' demographic and clinical baseline characteristics. If applicable, reference values are reported between parentheses.

\begin{tabular}{lc}
\hline Characteristic & Mean (SD) \\
\hline Age [years] & $64.3(8.0)$ \\
DXA T-score at lumbar spine ${ }^{\mathrm{a}}$ & $-2.4(1.6)$ \\
$\quad$ Normal BMD [n; \%] & $1(7.7 \%)$ \\
Osteopenic [n; \%] & $5(38.5 \%)$ \\
Osteoporotic [n; \%] & $7(53.8 \%)$ \\
Presence of VF [n; \%] & $5(41.7 \%)$ \\
25(OH)D [nmol/L] (15-162) & $68.9(28.3)$ \\
25(OH)D <50 nmol/L [n;\%] & $4(28.6 \%)$ \\
25(OH)D <75 nmol/L [n;\%] & $7(50.0 \%)$ \\
Calcium [mmol/L] (2.10-2.55) & $2.45(0.12)$ \\
PTH [pmol/L] (1.2-7.1) & $3.54(1.63)$ \\
TSH [mU/L]c (0.4-4.3) & $2.0(0.7-18.8)$ \\
Hypercalcemia [n; \%] & $0(0.0 \%)$ \\
Hyperthyroidism [n; \%] & $0(0.0 \%)$ \\
Hyperparathyroidism [n; \%] & $0(0.0 \%)$ \\
\hline
\end{tabular}

Values are reported as means and their standard deviations (SD) unless stated otherwise.

a From one patient no DXA data was available.

${ }^{b}$ VFA not performed in two patients.

${ }^{\mathrm{C}}$ Not normally distributed. Median and range are reported.

Abbreviations: DXA = dual-energy X-ray absorptiometry; $\mathrm{BMD}=$ bone mineral density; $\mathrm{VF}=$ vertebral fracture; $25(\mathrm{OH}) \mathrm{D}=25$-hydroxyvitamin $\mathrm{D} ; \mathrm{PTH}=$ parathyroid hormone; $\mathrm{TSH}=$ thyroid-stimulating hormone.

\section{Changes in cortical and trabecular micro-architecture}

No significant changes were observed in the trabecular and cortical microarchitectural parameters at the contra-lateral side during two years post-fracture.

\section{Changes in bone mechanical properties}

Similar to cortical BMD, also in compressive failure load (F.Ult) as well as stiffness in compression (Scomp) and bending (Sbend) an initial twelve-week period without change was followed by a decline, which led to a change from baseline at twoyears post-fracture of $-5.6 \%(p=0.007),-5.1 \%(p=0.014)$ and $-6.0 \%(p=0.029)$, respectively. Torsional stiffness showed a comparable pattern, but the $-2.5 \%$ change from baseline at two years post-fracture was not significant $(p=1.000)$. Separate analysis of the two-year changes in the patients who were compliant with anti-resorptive treatment, i.e. alendronate or denosumab, that started three months post-fracture $(n=5)$ and the patients who did not receive or were not compliant with anti-resorptive treatment $(n=6)$, showed a decreasing Scomp in patients who did not receive or were not compliant with alendronate treatment and in the patient treated with denosumab (Figure 1, right panel), while Scomp 
tended to decrease less in the patients who were compliant with alendronate treatment that was started at three months post-fracture.
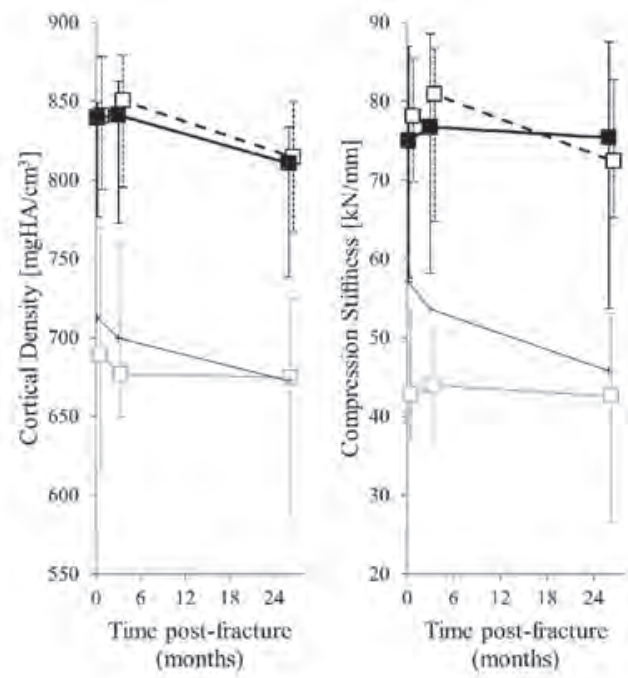

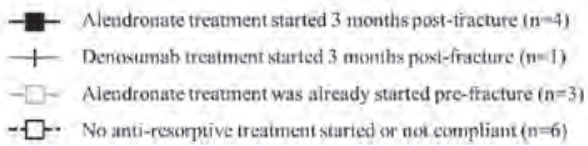

Figure 1 Median and 25\% and 75\% percentiles of cortical density (left) and compression stiffness (right) at the contra-lateral radius at baseline, three months and two years after a fracture in patients who started with alendronate (dark thick straight line) or denosumab (dark thin straight line) treatment at three months post-fracture, patients who were already started with alendronate treatment pre-fracture (light straight line), and patients who did not start or were not compliant to anti-resorptive treatment (dotted line).

\section{Changes in markers of bone formation and resorption}

Markers of bone formation (PINP) and resorption (ICTP) as well as the percentage change from baseline at each visit are presented in Table 3. During the first three months post-fracture, no significant changes were observed in both markers. At two years post-fracture, a significant decrease from baseline was observed in PINP (-50.9\%, $p=0.010)$, but not in ICTP $(-8.0 \%, p=1.000)$. A similar pattern was observed in patients who were compliant to alendronate treatment started at three months post-fracture and in patients who were not compliant or were not treated with alendronate. The patient who was treated with denosumab injections showed a clear decrease in levels of both PINP and ICTP (Figure 2). 


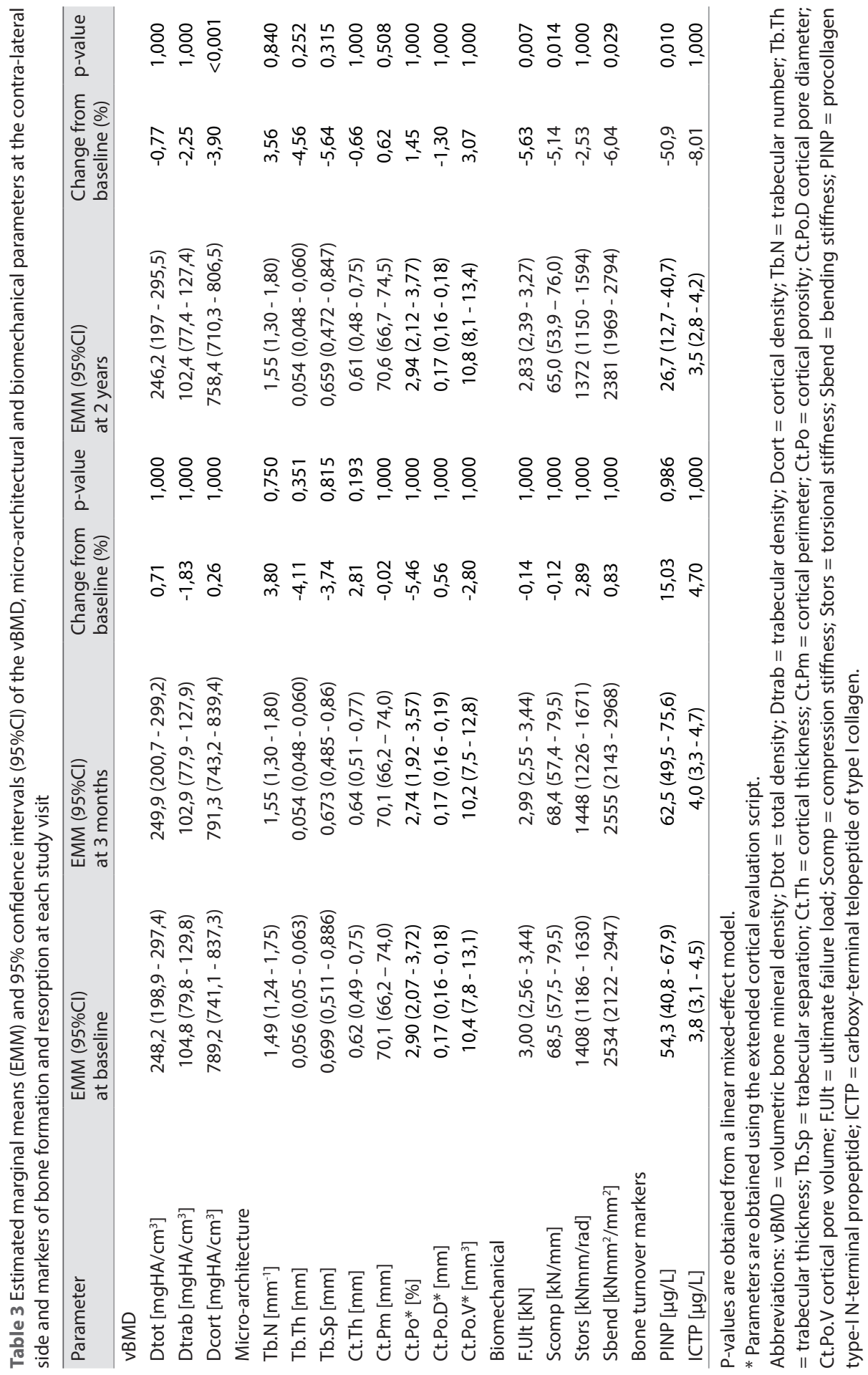




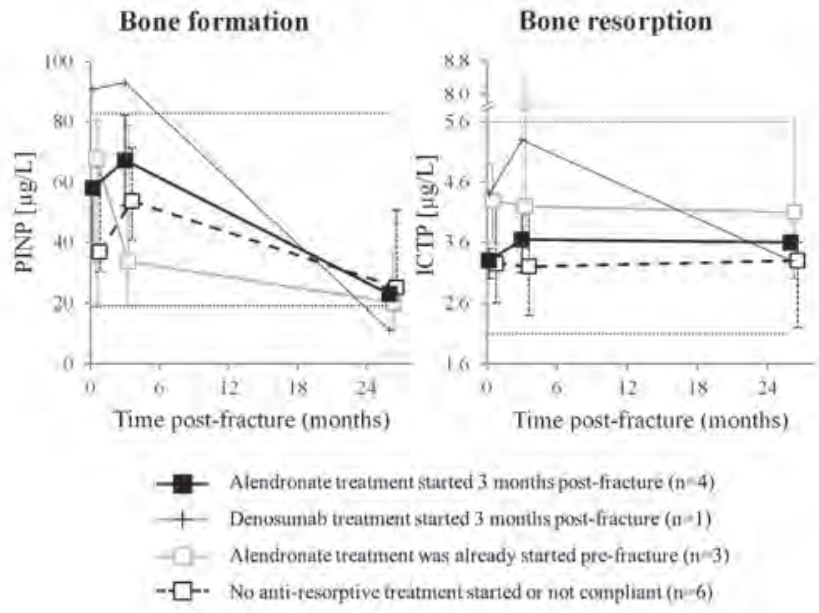

Figure 2 Median and 25\% and 75\% percentiles of bone formation and resorption markers at baseline, three months and two years post-fracture for patients who started with alendronate (dark thick straight line) or denosumab (dark thin straight line) treatment at three months post-fracture, patients who were already started with alendronate treatment pre-fracture (light straight line), and patients who did not start or were not compliant to anti-resorptive treatment (dotted line). The thin dotted lines mark the lower and upper reference limits as reported by the manufacturer from the analysis kits.

\section{Discussion}

This study showed a significant decrease of cortical VBMD and bone strength and stiffness at the contra-lateral distal radius two years after a stable distal radius fracture.

To our knowledge, there are only a few longitudinal studies that looked into bone changes at the contra-lateral side after a distal radius fracture. In these studies, no changes were observed in aBMD measured by DXA at the contra-lateral side ${ }^{8,9}$, and thus our finding that we did not observe a change in total VBMD is consistent with current literature. With $\mathrm{HRpQCT}$, however, it was possible to divide the total region in a cortical and trabecular compartment and analyze these compartments separately. We then found a decrease in cortical, but not in trabecular density. Also we did not find changes in cortical or trabecular micro-architecture.

Compared to literature, the two-year decline of $3.9 \%$ in cortical vBMD and $>5.0 \%$ in the biomechanical parameters at the contra-lateral side after a forearm fracture appeared to be higher than expected during normal aging. A recent prospective HRpQCT study on age- and gender-related changes in vBMD, micro-architecture 
and bone strength found an annual change in cortical VBMD and failure load of $-0.4 \%$ and $-0.9 \%$, respectively, in the group consisting of post-menopausal women $(n=51) .{ }^{19}$ The control group $(n=73)$ in a large HRpQCT intervention study on the effect of odanacatib showed a two-year decrease in cortical vBMD and estimated bone strength , of $-1.65 \%$ and $-1.61 \%$, respectively. ${ }^{20}$ Also the control group ( $n$ $=82$ ) in another large HRpQCT intervention study on the effect of alendronate and denosumab showed a one-year decrease in cortical BMD of $-1.5 \%{ }^{21}$, and in a small randomized controlled HRpQCT study on the effect of alendronate ${ }^{22}$, cortical vBMD tended to decrease and estimated failure load and stiffness in compression significantly decreased with approximately $3 \%$ in the control group $(n=13)$ during the two-year follow-up. Therefore, the results in our study suggest that there is a higher rate of bone loss in the cortical region in post-menopausal women with a distal radius fracture. Interestingly, a higher than expected decline in BMD was also observed after hip fractures by Magaziner et al. ${ }^{4,5}$ After hip fractures, the contralateral side is often immobilized as well due to a period of bedrest, which could attribute to the higher decline. However, this is not the case for forearm fractures and one could even argue that the non-fractured forearm is used more than in the pre-fracture situation.

The greater than expected declines in cortical vBMD and bone strength are particularly surprising, since it has been shown that alendronate and, to a larger extent, denosumab increase BMD by decreasing bone resorption, and secondary also bone formation. ${ }^{21-24}$ However, in two studies, patients with a fracture after age 50 were excluded. ${ }^{21,22}$

Whereas levels of bone turnover markers decreased in the patient being treated with denosumab, levels of bone resorption marker ICTP remained unchanged and levels of bone formation marker PINP decreased by $50 \%$ in patients with or without alendronate treatment. This could indicate uncoupling of bone resorption and formation in these patients, which is in line with the observed loss in cortical vBMD. The results of the bone turnover markers, however, should be interpreted in the context of fracture healing. Whereas the decreased bone formation is compatible with healing, i.e. the initial increase in PINP due to callus formation has decreased to low-normal at two years post-fracture, the unchanged bone resorption is difficult to explain. Still, non-compliance can be an issue ${ }^{25}$ for alendronate, but not for denosumab. Other explanations include persistent bone resorption of the callus, or another underlying yet unidentified metabolic bone disorder, associated with a recent fracture. Further elucidation of this process needs to be addressed in future studies. 
Given the precision error of the technique to assess cortical vBMD is approximately $1 \%{ }^{14,26,27}$, individual changes in cortical BMD need to exceed the least significant change (LSC) of $\pm 2.77 \%$ to be considered true changes. Since the two-year decline in cortical vBMD in all but one subject was larger than the LSC, we can indeed conclude that cortical vBMD truly decreased. The precision of the technique to assess the biomechanical parameters, however, is slightly lower with $3.6 \%{ }^{27}$, and hence the LSC for the biomechanical parameters is $\pm 10 \%$. A two-year decline in compression stiffness larger than $10 \%$ was observed in only four subjects, and in the remaining ten subjects the two-year decline in compression stiffness could not be considered true change. On a group level, however, the two-year decline in the biomechanical parameters is still significant.

The finding that the decrease in cortical density was accompanied by a decrease in the biomechanical parameters is not surprising, given the fact that the cortex carries a large proportion of the loads at the distal radius. ${ }^{28,29}$ Although also intracortical porosity is a good predictor for bone strength ${ }^{30-32}$, no significant changes in cortical porosity were found in our study. However, this might be a limitation of the resolution of the currently used HRpQCT device, which is not sufficient to capture small pores. Additionally, the precision of the currently used HRpQCT devices to assess cortical porosity is rather poor, i.e. RMS-CV\% of more than $10 \% .^{15,27,33}$ Future research on cortical porosity after a fracture should, therefore, make use of the second generation HRpQCT scanners, which have a higher spatial resolution.

\section{Limitations}

Our study has several limitations that are noteworthy. First of all, due to the exploratory nature of this pilot study a small number of subjects was included and, hence, we were limited in our statistical analyses. Also, we were not able to include a control group so it was not possible to make a comparison with subjects that did not fracture their wrist. Third, although not the purpose of this study, we did not longitudinally monitor bone mineral density at other sites than the distal radius, e.g. DXA of lumbar spine or hip, which could have provided more insight into the cortical bone loss. Fourth, we could not perform HRpQCT measurements between three months and two years post-fracture due to the time passed before we received approval from our ethical committee for an extension on the initial research protocol, in which a follow-up up to three months post-fracture was approved. Last, due to a change in the study protocol the HRpQCT scans at the two-year visit consisted of two consecutive stacks of 110 slices each. As a result of patient motion during scanning, these stacks might not be aligned perfectly which could lead to an underestimation of bone strength and stiffness. However, 
we discarded the measurements of insufficient quality in our linear mixed-effect model and therefore the shift between stacks should be minimal, thus leaving less room for errors. Moreover, not discarding the measurements of insufficient quality led to the same conclusions (data not shown).

\section{Conclusion}

We conclude that in post-menopausal women a stable fracture at the distal radius is associated with accelerated cortical bone loss and concomitant reduction of bone strength but not with micro-architectural changes at the contra-lateral distal radius, even in the presence of adequate anti-resorptive treatment.

\section{Acknowledgements}

The Authors would like to thank Frans Heyer for collection of the venous blood samples.

\section{Disclosures}

This study was supported by a grant from the Weijerhorst Foundation (WH-2).

B. van Rietbergen is a consultant for Scanco Medical AG.

J.J. Arts is a board member of workgroup Biotechnology of the Dutch Orthopedic Association (NOV) and board member Dutch Society for Biomaterials and Tissue Engineering.

P.C. Willems is a board member of the Dutch Spine Society (association of spine surgeons). 


\section{References}

1. van der Poest Clement, E. et al. Alendronate in the prevention of bone loss after a fracture of the lower leg. J Bone Miner Res 17, 2247-55 (2002).

2. Veitch, S.W. et al. Changes in bone mass and bone turnover following tibial shaft fracture. Osteoporos Int 17, 364-72 (2006).

3. Fox, K.M. et al. Loss of bone density and lean body mass after hip fracture. Osteoporos Int 11, 31-5 (2000).

4. Reider, L. et al. Women with hip fracture experience greater loss of geometric strength in the contra-lateral hip during the year following fracture than age-matched controls. Osteoporos Int 21, 741-50 (2010).

5. Magaziner, J. et al. Women with hip fracture have a greater rate of decline in bone mineral density than expected: another significant consequence of a common geriatric problem. Osteoporos Int 17, 971-7 (2006).

6. Karlsson, M. et al. Changes of bone mineral mass and soft tissue composition after hip fracture. Bone 18, 19-22 (1996).

7. Wolff, J. Das Gesetz der Transformation der Knochen. Verlag von August Hirschwald (1892).

8. van der Poest Clement, E., Patka, P. Vandormael, K., Haarman, H. \& Lips, P. The effect of alendronate on bone mass after distal forearm fracture. J Bone Miner Res 15, 586-93 (2000).

9. Ingle, B.M., Hay, S.M., Bottjer, H.M. \& Eastell, $R$. Changes in bone mass and bone turnover following distal forearm fracture. Osteoporos Int 10, 399-407 (1999).

10. de Jong, J.J. et al. Assessment of the healing process in distal radius fractures by highresolution peripheral quantitative computed tomography. Bone 64C, 65-74 (2014).

11. de Jong, J.J. et al. Fracture Repair in the Distal Radius in Post-Menopausal Women: A Follow-Up Two Years Post-Fracture Using HRpQCT. J Bone Miner Res 31, 1114-22 (2016).

12. de Jong, J.J. et al. Effect of a Cast on ShortTerm Reproducibility and Bone Parameters Obtained from HRPQCT Measurements at the Distal End of the Radius.J Bone Joint Surg Am 98, 356-62 (2016).
13. Pialat, J.B., Burghardt, A.J., Sode, M., Link, T.M. \& Majumdar, S. Visual grading of motion induced image degradation in high-resolution peripheral computed tomography: impact of image quality on measures of bone density and microarchitecture. Bone 50, 111-8 (2012).

14. Boutroy, S., Bouxsein, M.L., Munoz, F. \& Delmas, P.D. In vivo assessment of trabecular bone micro-architecture by high-resolution peripheral quantitative computed tomography. J Clin Endocrinol Metab 90, 6508-15 (2005).

15. Burghardt, A.J., Buie, H.R., Laib, A., Majumdar, S. \& Boyd, S.K. Reproducibility of direct quantitative measures of cortical bone micro-architecture of the distal radius and tibia by HRpQCT. Bone 47, 519-28 (2010).

16. Pistoia, W. et al. Estimation of distal radius failure load with micro-finite element analysis models based on three-dimensional peripheral quantitative computed tomography images. Bone 30, 842-8 (2002).

17. Dalzell, N. et al. Bone micro-architecture and determinants of strength in the radius and tibia: age-related changes in a populationbased study of normal adults measured with high-resolution pQCT. Osteoporos Int 20, 1683-94 (2009).

18. Bours, S.P., van den Bergh, J.P., van Geel, T.A. $\&$ Geusens, P.P. Secondary osteoporosis and metabolic bone disease in patients 50 years and older with osteoporosis or with a recent clinical fracture: a clinical perspective. Curr Opin Rheumatol 26, 430-9 (2014).

19. Shanbhogue, V.V., Brixen, K. \& Hansen, S. Age- and Sex-Related Changes in Bone Micro-architecture and Estimated Strength. A Three-Year Prospective Study Using HRpQCT. J Bone Miner Res 31, 1541-9 (2016).

20. Cheung, A.M. et al. Effects of odanacatib on the radius and tibia of postmenopausal women: improvements in bone geometry, micro-architecture, and estimated bone strength. J Bone Miner Res 29, 1786-94 (2014).

21. Seeman, E. et al. Micro-architectural deterioration of cortical and trabecular bone: differing effects of denosumab and alendronate. J Bone Miner Res 25, 1886-94 (2010).

22. Burghardt, A.J. et al. A longitudinal HRpQCT study of alendronate treatment in postmenopausal women with low bone density: Relations among density, cortical and trabecular micro-architecture, biomechanics, and bone turnover. J Bone Miner Res 25, 2558-71 (2010). 
23. Rizzoli, R. et al. Effects of strontium ranelate and alendronate on bone microstructure in women with osteoporosis. Results of a 2-year study. Osteoporos Int 23, 305-15 (2012).

24. Tsai, J.N. et al. Comparative effects of teriparatide, denosumab, and combination therapy on peripheral compartmental bone density, micro-architecture, and estimated strength: the DATA-HRpQCT Study. J Bone Miner Res 30, 39-45 (2015).

25. Klop, C. et al. Long-term persistence with anti-osteoporosis drugs after fracture. Osteoporos Int 26, 1831-40 (2015).

26. MacNeil, J.A. \& Boyd, S.K. Improved reproducibility of high-resolution peripheral quantitative computed tomography for measurement of bone quality. Med Eng Phys 30, 792-9 (2008).

27. Ellouz, R. et al. Challenges in Iongitudinal measurements with HRpQCT: evaluation of a $3 D$ registration method to improve bone micro-architecture and strength measurement reproducibility. Bone 63, 14757 (2014).

28. MacNeil, J.A. \& Boyd, S.K. Load distribution and the predictive power of morphological indices in the distal radius and tibia by highresolution peripheral quantitative computed tomography. Bone 41, 129-37 (2007).
29. Vilayphiou, N. et al. Finite element analysis performed on radius and tibia $\mathrm{HRPQCT}$ images and fragility fractures at all sites in postmenopausal women. Bone 46, 1030-7 (2010).

30. Bala, Y., Zebaze, R. \& Seeman, E. Role of cortical bone in bone fragility. Curr Opin Rheumatol 27, 406-13 (2015).

31. Wachter, N.J. et al. Prediction of cortical bone porosity in vitro by microcomputed tomography. Calcif Tissue Int 68, 38-42 (2001).

32. Augat, P. \& Schorlemmer, S. The role of cortical bone and its microstructure in bone strength. Age Ageing 35 Suppl 2, ii27-ii31 (2006).

33. Kawalilak, C.E., Johnston, J.D., Cooper, D.M. Olszynski, W.P. \& Kontulainen, S.A. Role of endocortical contouring methods on precision of HRpQCT-derived cortical microarchitecture in postmenopausal women and young adults. Osteoporos Int 27, 789-96 (2016). 



$$
x
$$




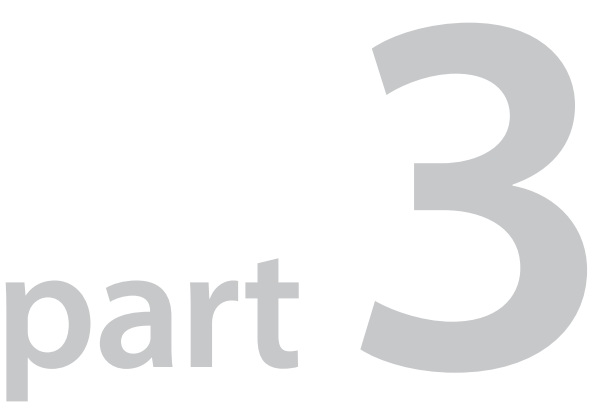

Applicability of HRpQCT in unstable distal radius fractures 


$$
\frac{b}{x}
$$




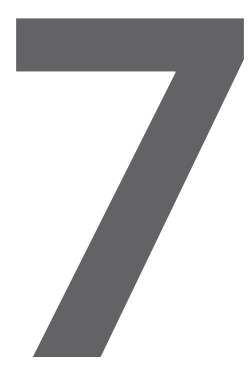

Feasibility of rigid 3D image registration of high-resolution peripheral quantitative computed tomography images of healing distal radius fractures 


\section{Abstract}

\section{Introduction}

For accurate analysis of bone formation and resorption during fracture healing, correct registration of follow-up onto baseline image is required. A per-fragment approach could improve alignment compared to standard registration based on the whole fractured region. In this this exploratory study, we tested the effect of fragment size and displacement on a per-fragment registration, and compared the results of this perfragment registration to the results of the standard registration in two stable and one unstable fracture(s).

\section{Methods}

To test the effect of fragment size and displacement, high-resolution peripheral quantitative computed tomography (HRpQCT) scans of three unfractured radii were divided into subvolumes. Different displacements in $\mathrm{x}-, \mathrm{y}$, or z-direction or rotations around each axis were applied, and each subvolume was registered onto the initial volume to realign it. Next, registration of follow-up onto baseline scan was performed in two stable and one unstable fracture. After coarsely aligning the follow-up onto the baseline scan, a more accurate registration was performed of the whole fracture and the standard registration, and of each fracture fragment separately. Alignment was checked using overlay images showing baseline, follow-up and overlap between these scans, and by comparing correlation coefficients between the standard and perfragment registration.

\section{Results}

Generally, subvolumes as small as $300 \mathrm{~mm}^{3}$ that were displaced up to $0.82 \mathrm{~mm}$ in $\mathrm{x}$ or y-direction, or up to $1.64 \mathrm{~mm}$ in z-direction could be realigned correctly. For the fragments of all fractures, correlation coefficients were higher after per-fragment registration compared to standard registration. Most improvement was found in the unstable fracture and one fragment of the unstable fracture did not align correctly.

\section{Conclusion}

This exploratory study showed that image registration of individual subvolumes, such as fracture fragments, is feasible in both stable and unstable fractures, and leads to better alignment of these fragments compared to an approach that is based on registration using the whole fractured region. This result is promising for additional analysis of bone formation and resorption in HRpQCT studies on fracture healing. 


\section{Introduction}

In medical image analysis, image registration is a commonly used method where two (or more) images are aligned by optimizing a similarity measure, which is often based on voxel intensity cross-correlation' or mutual information. ${ }^{2,3}$ In this way, it is possible to gain valuable information that is conveyed in more than one image, e.g. images from different time points, different modalities or distinct viewpoints. ${ }^{4}$ Usually, one image is called the 'fixed' or reference image, which is the image on which the second image, called the 'moving' image, is registered.

Image registration procedures have been developed for almost every tissue and organ. For bone, image registration has been used for various purposes, including improving the reproducibility of high-resolution computed tomography (CT) derived bone parameters ${ }^{5}$, strain mapping of loaded bone ${ }^{6}$, and for the analysis of sites of bone formation and resorption at the tibia ${ }^{7}$ and finger joints. ${ }^{8}$ For fractured bone, Lynch et al. applied image registration to longitudinal CT images of healing distal radius fractures to assess changes in $\mathrm{CT}$ image intensity within the fracture gap during the healing process ${ }^{9}$, and Tassani et al. used image registration to automatically detect the fracture zone in microCT images of trabecular bone. ${ }^{10}$ In the aforementioned studies, mostly a rigid image registration procedure was used because bone is a rigid tissue., ${ }^{5,-10}$ Zwahlen et al., however, used a deformable (non-rigid) image registration approach because of local deformation of individual trabeculae. ${ }^{6}$

Compared to clinical CT used by Lynch et al. ${ }^{9}$, high-resolution peripheral quantitative $\mathrm{CT}$ (HRpQCT) has a higher spatial resolution which allows for a more detailed assessment of the bone changes at the fracture site. We recently used HRpQCT to study the healing process of distal radius fractures treated by closed reduction and cast immobilization, and we found significant changes in volumetric bone mineral density (VBMD), micro-architecture and biomechanical parameters were found during the healing process. ${ }^{11,12}$ In addition, superposition of these sequential HRPQCT images using a rigid 3D image registration in a way similar to Christen et al. did in normal bone, would allow the study of changes of bone formation and resorption, which are intense metabolic processes during fracture healing and may be affected by certain anti-osteoporosis drugs. ${ }^{13}$ Applied to preliminary data from a pilot study by Bours et al., this method indeed showed interesting results in a simple, stable fracture (Figure 1). ${ }^{14}$ However, although all distal radius fractures in our study were treated as stable fractures, some fractures might be less stable than expected and fracture fragments might move relative to 
each other in between two HRpQCT scans. This may lead to erroneous results when the HRpQCT images are superimposed by the rigid 3D registration procedure in order to visualizing bone formation and resorption, because moved fragments may wrongly be qualified as formed or resorbed bone. Although deformable image registration might be able to correct for such relative movement between fragments, the individual fragments are still mainly composed of rigid bone tissue and, hence, a rigid image registration procedure seems more appropriate.
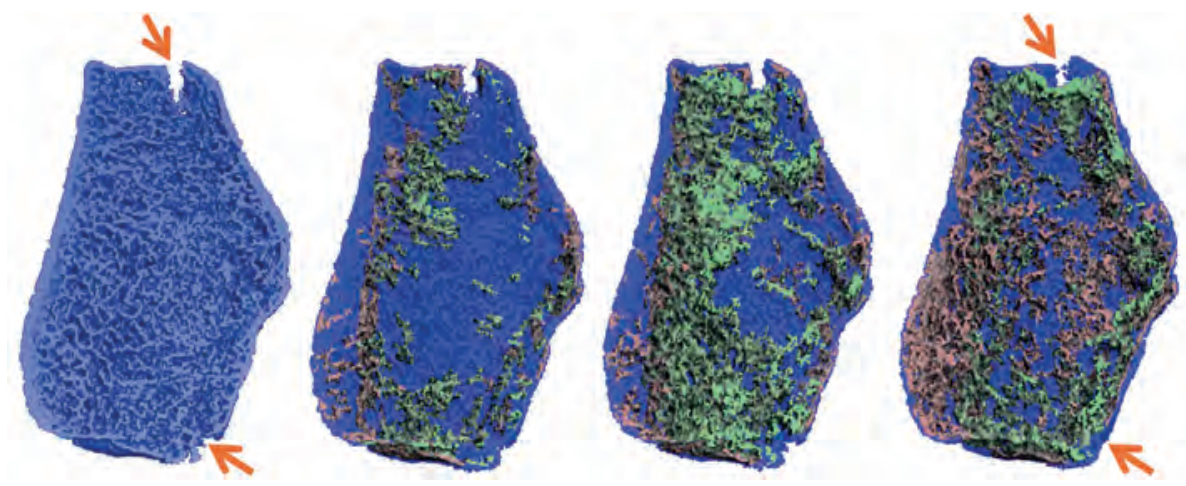

Figure 1 Sites of bone formation and resorption during healing of a stable distal radius fracture. 3D HRpQCT images of a stable, simple distal radius fracture at 9 days (baseline) and at follow-up 26, 44 and 87 days post-fracture showing regions of bone formation (green), resorption (red) and no change (blue). Images were obtained by superposition of the follow-up images over the baseline image. Cortical fracture locations (indicated by arrows) are bridged at 87 days post-fracture.

To limit the amount of wrongly qualified formed or resorbed bone in HRpQCT images of healing distal radius fractures, we propose to, after a pre-registration, select individual fragments in the moving image and register them separately into the fixed image using rigid 3D image registration. This approach, however, raises several questions that need to be addressed. First of all, the separate fragments are smaller than the scanned bone region itself. It is currently unclear to what extend the registration is affected by using only a subvolume of the moving image as input, instead of using the whole bone region. Second, changes related to the fracture healing process will occur which are larger than changes due to aging or medication and thus the similarity between the fragments can be low, even if correctly registered. To address these issues, we first tested the proposed method using a subset of HRpQCT images of a reproducibility study. Then, we applied our method to stable fractures that were minimally displaced and to a fracture where secondary displacement had occurred. 


\section{Materials and Methods}

\section{Subjects and image data sets}

Two datasets were used in this study. The first dataset consisted of HRpQCT scans of fifteen healthy individuals with ages ranging from 21 to 47 years and was presented earlier in the reproducibility study of Boutroy et al..$^{15}$ This dataset was also previously used by Christen et al. to study the reproducibility of bone apposition and resorption quantification in healthy subjects. In the present study, three subjects were randomly selected from this dataset to test the effect of size versus displacement on the fracture registration algorithm. Informed consent was obtained from all participants and the reproducibility study was approved by an independent Ethics Committee (Comité Consultatif de Protection des Personnes dans la Recherche Biomédicale de Lyon).

The second dataset consisted of HRPQCT scans that were made during the first twelve weeks of fracture healing in post-menopausal women with a distal radius fracture who were treated by closed reduction and cast immobilization. Image acquisition has been described previously ${ }^{11}$, but briefly, an $18 \mathrm{~mm}$-long region covering the fracture was scanned by HRpQCT at one to two, three to four, six to eight and twelve weeks post-fracture using standard in vivo settings (tube voltage $60 \mathrm{kVp}$, tube current $900 \mu \mathrm{A}$, integration time $100 \mathrm{~ms}$ ). Using an isotropic voxelsize of $82 \mathrm{~mm}$, each HRpQCT measurement thus resulted in transverse 220 slices. After testing the fracture registration algorithm, it was applied onto three fractures that were selected from this dataset to test if a registration based on fragments improved the registration in these fragments. Since movement is most likely to occur during the early phases of fracture healing, when the fragments are not stabilized yet, baseline images at one to two weeks post-fracture and followup images at three to four weeks post-fracture were used. Furthermore, HRpQCT images of the fractures were selected on having no or minimal motion artifacts. ${ }^{16}$ Informed consent was obtained from all participants and the fracture healing study was approved by the local Medical Ethics Committee (NTR3821).

\section{Description of the fracture registration algorithm}

For a correct analysis of sites of bone formation and resorption as calculated from two consecutive HRpQCT scans, a proper registration of the two scans is required. The rigid 3D registration procedure (Image Processing Language version 5.16/regis 1.09b, Scanco Medical AG, Brütissellen, Switzerland) used in the present study can be divided into two parts:

1) A pre-registration to coarsely align the follow-up image onto the baseline image using solid volumes of the periosteal contours of these images 
2) Fine registration per fragment to precise register each fragment from the follow-up image onto the baseline image using the gray-scale images within the contours of the fragments

1) Pre-registration

Because the orientation of the fractured radius may differ between two HRpQCT scans, a pre-registration was required to coarsely align the followup image onto the baseline image. This pre-registration was based on solid volumes within the periosteal contours in the two images. The periosteal contours were created using the standard semi-automatic method using edge detection. Since rotations of the radius around the $x$ - and $y$-axis are restricted due to fixation of the forearm during scanning procedure, the preregistration started by rotating the mask of the follow-up image, that has been downscaled ten times, in steps of 22.5 degrees around the z-axis to save time. The angle at which the best overlap was found was then used as the starting position for a registration at four times downscaling in which the follow-up masks was iteratively translated along and rotated around each axis until the best overlap was found.

2) Fine registration per fragment

After this course alignment, a fine 3D rigid registration of the individual fragments in the follow-up image onto the baseline image was performed. The rotations and translations found in the pre-registration were used as starting position for each fragment in the fine registration. Since each fragment is supposed to be already more or less in place, this fine registration was performed using grayscale images that were first downscaled four times, and finally using the original image resolution. In this fine registration step, both the total follow-up and baseline images were used as input images, but only information within the volume of interest (VOI) in each image was counted in calculation of best overlap. The VOI in the moving image was the contoured fragment, and the VOI in the fixed image the region within the periosteal contour.

\section{Effect of fragment size, offset and step-size}

To test how well small fragments of different size can be registered, the 3D volume of each radius scan was divided into two, four and eight subvolumes (Figure 2). Each subvolume was then shifted from its original position by 10 or 20 voxels $(0.82$ and $1.64 \mathrm{~mm}$, respectively) in the $x_{-}, y_{-}$, or $z$-direction, or rotated around the $x-, y-$, or z-axis by 0.1 or 0.2 rad (5.7 and 11.5 degrees, respectively). Next, each shifted/ 
rotated subvolume was registered onto the initial, total scan to see if it could be registered correctly. With the registration a simplex search algorithm was used that performs translation/rotation trial steps of a typical size. For periodic structures, such as bone, it was expected that the translation step size should be close to the size of the trabecular separation, to avoid ending at a local minimum. To test the effect of the typical step-size in each iteration, the same procedure described above was repeated for step-sizes of one and two times the mean trabecular separation per scan. To quantify the agreement at each iteration, a correlation coefficient (CC) was calculated according to equation $1::^{17}$

$$
c c=\frac{{ }_{i=1}^{n v} v_{i} w_{i}}{\sqrt{{ }_{i=1}^{n v} v_{i} v_{i}{ }_{i=1}^{n v} w_{i} w_{i}}}
$$

where $v_{i}$ is the value of voxel $i$ in image 1, i.e. the fixed image; $w_{i}$ the value of a corresponding voxel in image 2 , i.e. the moving image, after translation/rotation using tri-linear interpolation; and $n v$ the number of voxels in the evaluated region. Since the image information per subvolume is exactly the same as in the initial image, a correlation coefficient of 1.000 indicates a perfect registration.
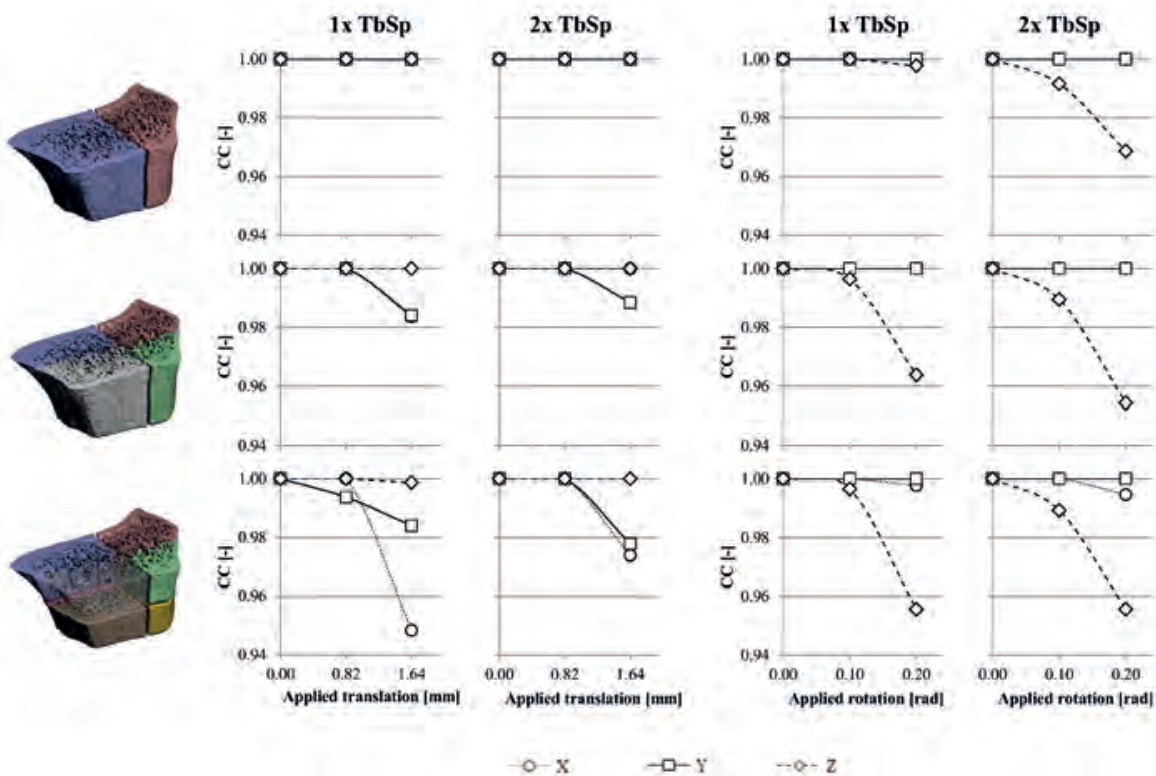

$-\mathrm{x}$

$-0-z$

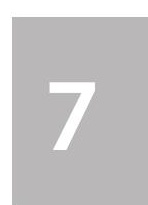

Figure 2 Subvolumes and corresponding correlation coefficients. Average correlation coefficients for registration of volumes with different sizes, using different step-sizes after different applied translations in $x-, y$ - and $z$-direction and after different applied rotations around the $x-, y$ - and $z$-axis. 


\section{Implementation onto healing fractures}

When the performance of the registration per part of different sizes, maximum allowed offset and the optimal step-size were known, the method was applied onto several fracture cases:'simple' fractures with two fragments that are relatively stable over time, and 'complex' fractures with three or more fragments that have moved relative to each other over time. To be able to study the expected beneficial effect of per-fragment registration, each scan of each fracture case was also registered using the standard registration approach which uses the whole bone region in the follow-up image in the registration.

Per fragment the result of the registration was determined qualitatively by checking overlay images. These overlay images were created by combining segmented 3D volumes of the fracture at baseline and the registered follow-up image, with voxels in the baseline volume set to value 1 (red) and in the follow-up volume set to value 2 (green). Thus, after combining these volumes, overlapping regions in the overlay image have value 3 (purple)..$^{18}$ The segmented 3D volumes were created from the gray-scale images by applying a Gaussian filter (sigma = 0.7 , width $=1.0$ voxel) to remove noise followed by thresholding (threshold $=120$ $\left.\mathrm{mgHA} / \mathrm{cm}^{3}\right)$. Furthermore, correlation coefficients ${ }^{17}$ within each fragment region were compared between the registrations based on the standard approach and the per-fragment approach to see whether the registration was improved.

\section{Results}

\section{Effect of fragment size, offset and step-size}

The average correlation coefficients for registration of the subvolumes of different sizes, using different step-sizes after different applied translations in $\mathrm{x}-, \mathrm{y}$ - and $z$-direction and after different applied rotations around the $x-, y$ - and $z$-axis are presented in Figure 2 . The average volumes \pm standard deviation (SD) of the onehalve, one-quarter and one-eight parts were $1200 \pm 130 \mathrm{~mm}^{3}, 596 \pm 108 \mathrm{~mm}^{3}$, and $298 \pm 74 \mathrm{~mm}^{3}$, respectively. The trabecular separation measured in the total volumes was $0.561 \mathrm{~mm}, 0.626 \mathrm{~mm}$, and $0.673 \mathrm{~mm}$.

Regarding the applied translations, a correct registration was possible after a translation of the one-halve parts of 0.82 and $1.64 \mathrm{~mm}$ in each direction, regardless of step-size. For one-quarter and one-eight parts, in general a correct registration was found after translations of 0.82 and $1.64 \mathrm{~mm}$ in the z-direction, but not in the $x$ - and $y$-direction: then the registration failed at translation of $1.64 \mathrm{~mm}$.

Regarding the applied rotations, a correct registration was found for rotations of the one-halve and one-quarter parts around the $x$ - and $y$-axis by 0.10 and 0.20 
radians, regardless of step-size, whereas registration failed after rotations around the z-axis. For one-eight parts, registration was correct for rotations of 0.1 and 0.2 radians around the $x$-axis and 0.1 radians around the $y$-axis, but failed for rotations of 0.2 radians around the $y$-axis and 0.1 and 0.2 radians around the $z$-axis, regardless of step-size.

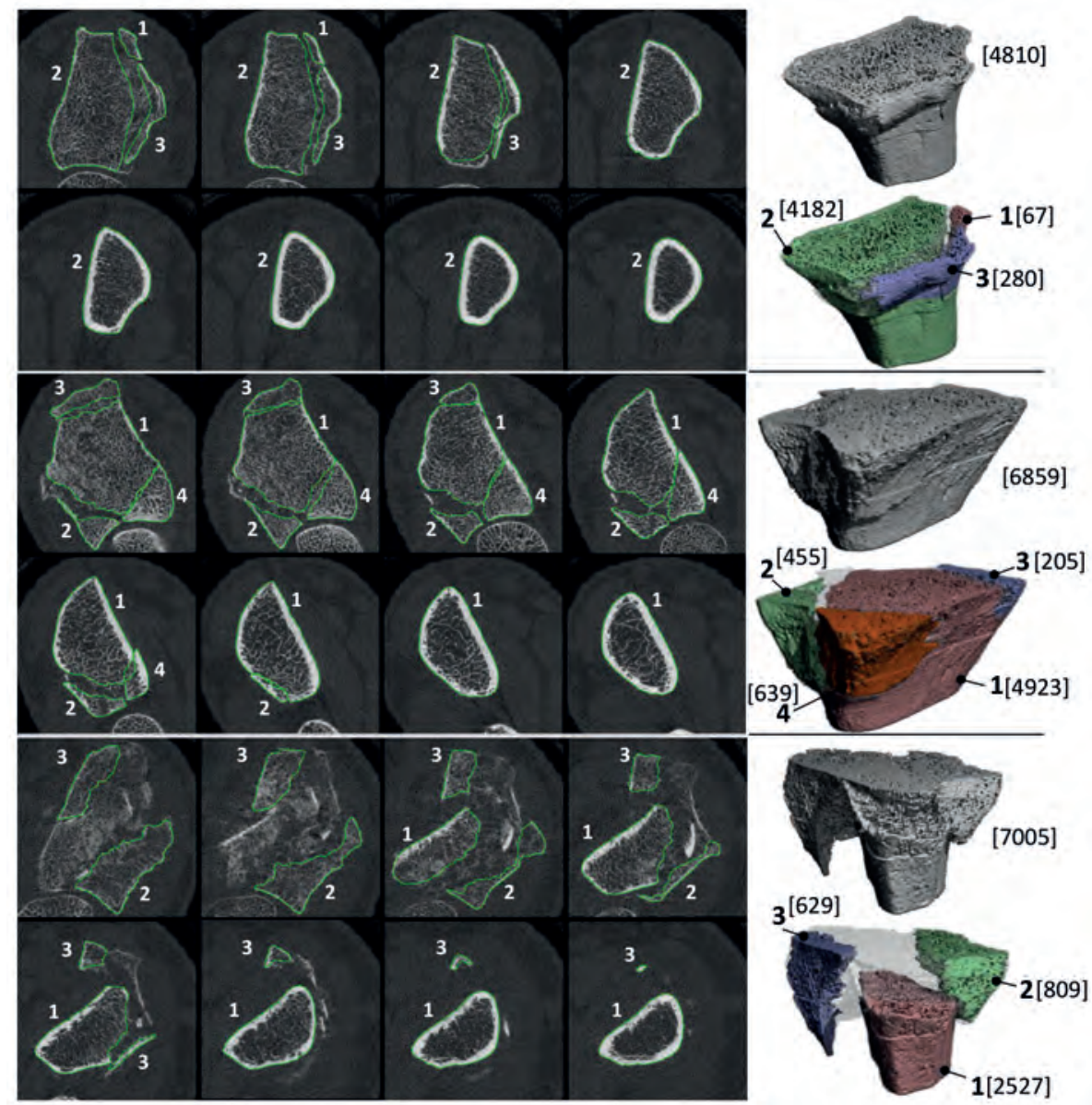

Figure 3 2D HRpQCT slices with contours (left) and 3D model (right) of the fragments in two stable fractures (top and middle) and a fracture with secondary displacement (bottom) that were registered in this study. The volume [mm3] per fragment as well as of the complete fracture is shown between brackets. 


\section{Application to fractures}

In Figure 3, 2D HRpQCT slices with the contoured fragments are shown, as well as 3D models of each fracture with the fragments visualized in different colors. In each fracture, one large volume could be defined, with volumes ranging from $2527 \mathrm{~mm}^{3}$ to $4923 \mathrm{~mm}^{3}$ covering $36 \%$ to $87 \%$, respectively, of the total volume. Additionally, two or three smaller fragments could be contoured per fracture and their volumes ranged from $67 \mathrm{~mm}^{3}$ to $809 \mathrm{~mm}^{3}$.

Overlay images and corresponding correlation coefficients resulting from the registration using the whole bone region in the follow-up scan, and from the registration per fragment are shown in Figure 4. The overlay images suggest that registration based on the whole bone region resulted in an (almost) correct registration in the two stable fractures but not for the unstable fracture. Corresponding correlation coefficients were $0.970,0.963$ and 0.909 , respectively. For all fragments in each fracture, the per-fragment registration resulted in increased correlation coefficients compared to registration based on the whole bone region. The largest improvement in correlation coefficient was observed in fragment 1 of the unstable fracture. Fragment 3 of the unstable fracture showed improved correlation coefficient, while the overlay image clearly showed a misalignment of this fragment after per-fragment registration.

\section{Discussion}

In this exploratory study, we showed for the first time that image registration of separate (fracture) fragments is feasible in both stable and unstable fractures, and leads to better alignment of these fragments compared to an approach that is based on registration using the whole bone region.

Not unexpected, correct realignment occurred more for larger parts than for smaller parts. In smaller parts, realigned was observed after an offset in the z-directions and applied rotations in the z-plane. Interestingly, this movement is likely similar to what happens in case of secondary displacement: the distal part of the fracture collapses over the proximal part in the longitudinal direction, with no or relatively little rotation in the transversal plane.

Based on our results, a step-size similar to the trabecular separation can be advised. This finding is not surprising, since most parts consist mainly of trabeculae. With step-sizes smaller than the trabecular separation, the registration might find a local minimum, hence leading to a non-optimal registration. By using a step-size 

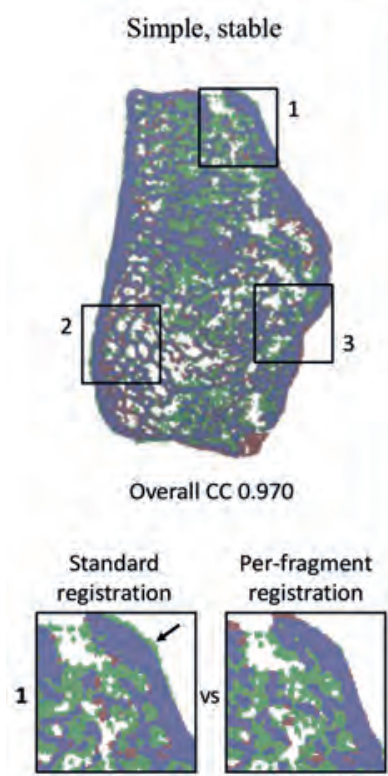

CC 0.955

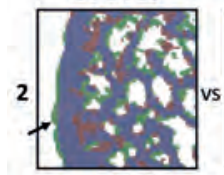

CC 0.969

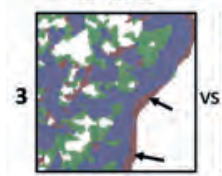

CC 0.968

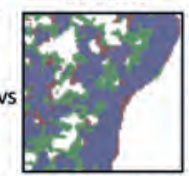

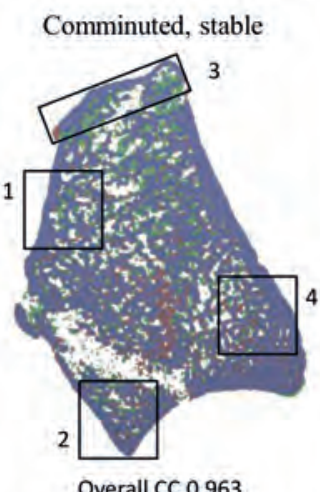

Overall CC 0.963

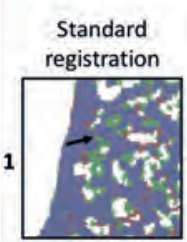

CC 0.966

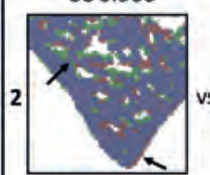

CC 0.952

CC 0.977

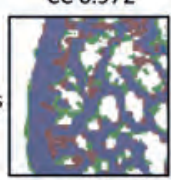

CC 0.970

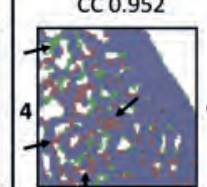

cc 0.957

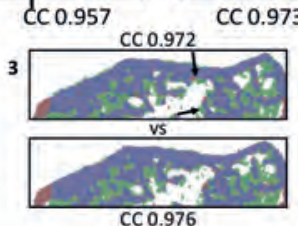

Comminuted, unstable
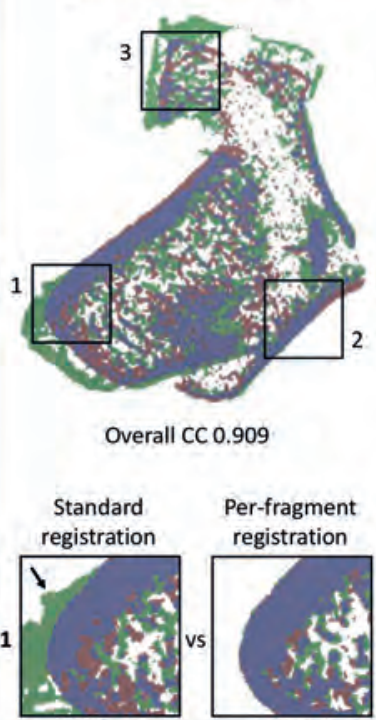

CC 0.914

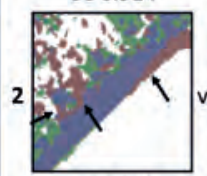

CC 0.969

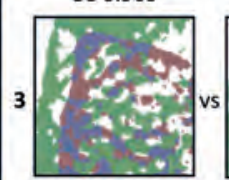

CC 0.897
CC 0.962

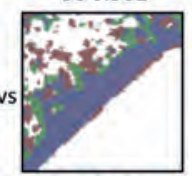

CC 0.970

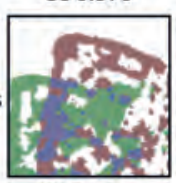

CC 0.901

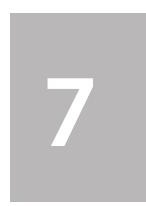

Figure 4 Overlay images of three fracture cases. Overlay image after standard registration of the whole bone region in the follow-up image (three to four weeks post-fracture) onto baseline (one to two weeks post-fracture) with corresponding correlation coefficients (top). Overlapping regions (purple) are shown as well as regions belonging to baseline (red) and follow-up (green). To check whether the perfragment registration improved the results compared to the standard registration, overlay images and corresponding correlation coefficients are presented for the same subregions obtained after standard and per-fragment registration. Arrows indicate locations of clear improvement after per-fragment registration. Correlation coefficients, which are reported below each fragment, are higher after perfragment registration than after standard registration. 
of at least the trabecular separation, the chance of finding a local minimum is less likely. If individual measurement of trabecular separation is not feasible in a practical setting, using a step-size is advised of approximately $0.7 \mathrm{~mm}$, which is the trabecular separation at the distal radius of osteoporotic and osteopenic postmenopausal women. ${ }^{15}$

In the fractured cases, alignment of the fragments was improved in all cases when the fragments were registered separately in comparison to when the whole bone region was used in the registration. The challenge is to determine for which fractures the per-fragment registration is required in order to obtain reliable results on bone apposition and resorption. It is expected that the stable fractures were sufficiently aligned using the standard registration approach to determine the amount of bone formation and resorption since correlation coefficient were already high and no major increases in correlation coefficients were observed after per-fragment registration. In the unstable fracture, however, a major improvement in alignment was observed for fragment 1. This indicates that unstable fractures can benefit most from the per-fragment registration as presented in this study.

On the other hand, per-fragment registration of fragment 3 in the unstable fracture did not result in a satisfactory alignment. An explanation can be found in the size of the fragment with respect to the displacement. Being the smallest fragment in the unstable fracture with a volume of $629 \mathrm{~mm}^{3}$, it had to overcome a distance of approximately $1 \mathrm{~mm}$ (13 voxels) in the $\mathrm{x}$-direction and $2 \mathrm{~mm}$ ( 24 voxels) in the $y$-direction. Since the one-halve volumes with a volume of approximately $600 \mathrm{~mm}^{3}$ failed to realign correctly after displacements in the $\mathrm{x}$ - or- $y$-direction of $>0.82 \mathrm{~mm}$ (10 voxels), this might indicate that the fragment was too small in relation to the displacement.

Although not quantitatively measured, feasible contouring of fracture fragments depends in our experience heavily on the presence and amount of low mineralized callus, the number of fragments, and the degree to which the fragments are interconnected. Therefore, fragments in HRpQCT scans that were made early post-fracture, i.e. at one to two weeks post-fracture, are less difficult to contour than in the HRpQCT scans made at three to four or even six to eight weeks post-fracture. At six to eight weeks post-fracture, a clear distinction between the fragments may be severely hindered by the amount of low mineralized callus that has formed at the fracture line. However, this might not be a problem: at the time this low mineralized tissue has formed, the fragments are not likely to move relative to each other as they have already been stabilized by the presence of callus. We therefore expect that only fragments in the scans that are made during the first four weeks post-fracture, need contouring. 
Double-stack (220 slices / $18 \mathrm{~mm}$ ) HRpQCT measurements were made to ensure that most of the fracture region was included. However, even despite including only measurements with no or very limited motion artifacts, a minor shift between the stacks is almost impossible to avoid. Also in our study, this was the case as can be seen by the edges that are visible in the 3D models in Figure 3. To improve alignment of fragments that are more than 110 slices $(9 \mathrm{~mm})$ long, it is advised to align the two stacks per measurement before registering volumes from the followup scan onto the baseline scan. Another option is to limit the registration to one stack, but this has the disadvantage that fragments may become very small and are thus more likely to end up misaligned.

A few limitations need to be addressed. In the present study, contouring of the fragments was done manually, which is subjective and may lead to low intra- and inter-operator reproducibility. Although exploratory, we have shown that separate registration of each fragment may improve the total registration in fractures. Future work should therefore focus on an automatic procedure for finding the fracture line and/or contouring different fragments. Second, we did not apply our approach to experimental data where fragment displacement could be controlled. While focusing on in vivo patient data, such an experiment would have been beyond the scope of the present study. Last, we only used HRpQCT scans of good quality. It is therefore unknown to what extent the registration is affected by the presence of motion artifacts.

\section{Conclusion}

This exploratory study showed that image registration of individual (fracture) fragments is feasible in both stable and unstable fractures, and leads to better alignment of these fragments compared to an approach that is based on registration using the whole fractured region. This result is promising for additional analysis of bone resorption and formation based on longitudinal HRpQCT images of healing fractures, for example in studies on the metabolic effects of anti-osteoporosis medication.

\section{Acknowledgements}

None. 


\section{References}

1. Collins, D.L., Neelin, P., Peters, T.M. \& Evans, A.C. Automatic 3D intersubject registration of MR volumetric data in standardized Talairach space. J Comput Assist Tomogr 18, 192-205 (1994).

2. Maes, F., Collignon, A., Vandermeulen, D., Marchal, G. \& Suetens, P. Multimodality image registration by maximization of mutual information. IEEE Trans Med Imaging 16, 187-98 (1997).

3. Wells, W.M., 3rd, Viola, P., Atsumi, H., Nakajima, S. \& Kikinis, R. Multi-modal volume registration by maximization of mutual information. Med Image Anal 1, 35-51 (1996).

4. Oliveira, F.P. \& Tavares, J.M. Medical image registration: a review. Comput Methods Biomech Biomed Engin 17, 73-93 (2014).

5. Ellouz, R. et al. Challenges in longitudinal measurements with HRPQCT: evaluation of a $3 D$ registration method to improve bone micro-architecture and strength measurement reproducibility. Bone 63, 14757 (2014).

6. Zwahlen, A. et al. Inverse finite element modeling for characterization of local elastic properties in image-guided failure assessment of human trabecular bone. J Biomech Eng 137 (2015).

7. Christen, P. et al. Bone remodelling in humans is load-driven but not lazy. Nat Commun 5, 4855 (2014).

8. Topfer, D. et al. Automated three-dimensional registration of high-resolution peripheral quantitative computed tomography data to quantify size and shape changes of arthritic bone erosions. Rheumatology (Oxford) 54, 2171-80 (2015).

9. Lynch, J.A. et al. Measurement of changes in trabecular bone at fracture sites using X-ray CT and automated image registration and processing. J Orthop Res 22, 362-7 (2004).

10. Tassani, S., Matsopoulos, G.K. \& Baruffaldi, F. 3D identification of trabecular bone fracture zone using an automatic image registration scheme: A validation study. J Biomech $\mathbf{4 5}$ 2035-40 (2012).
11. de Jong, J.J. et al. Assessment of the healing process in distal radius fractures by highresolution peripheral quantitative computed tomography. Bone 64C, 65-74 (2014).

12. de Jong, J.J. et al. Fracture Repair in the Distal Radius in Post-Menopausal Women: A Follow-Up Two Years Post-Fracture Using HRpQCT. J Bone Miner Res 31, 1114-22 (2016).

13. Hegde, V., Jo, J.E., Andreopoulou, P. \& Lane, J.M. Effect of osteoporosis medications on fracture healing. Osteoporos Int 27, 861-71 (2016).

14. Bours, $S$. et al. Fracture Healing in Postmenopausal Women with a Distal Radius Fracture Monitored by HighResolution Peripheral Quantitative Computer Tomography (HRpQCT): A Pilot Study [abstract]. J Bone Miner Res 27 (Suppl 1) (2012).

15. Boutroy, S., Bouxsein, M.L., Munoz, F. \& Delmas, P.D. In vivo assessment of trabecular bone micro-architecture by high-resolution peripheral quantitative computed tomography. J Clin Endocrinol Metab 90, 6508-15 (2005).

16. Pialat, J.B., Burghardt, A.J., Sode, M., Link, T.M. \& Majumdar, S. Visual grading of motion induced image degradation in high-resolution peripheral computed tomography: impact of image quality on measures of bone density and microarchitecture. Bone 50, 111-8 (2012).

17. Verhulp, E., van Rietbergen, B. \& Huiskes, R. A three-dimensional digital image correlation technique for strain measurements in microstructures. J Biomech 37, 1313-20 (2004).

18. Schulte, F.A., Lambers, F.M., Kuhn, G. \& Muller, R. In vivo micro-computed tomography allows direct three-dimensional quantification of both bone formation and bone resorption parameters using timelapsed imaging. Bone 48, 433-42 (2011). 



$$
\frac{b}{x}
$$




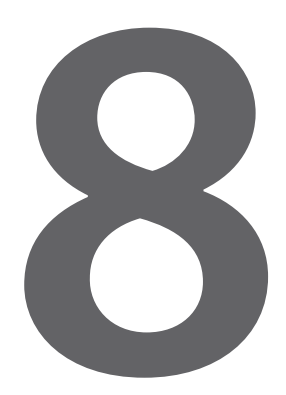

Distal radius plate of CFR-PEEK has minimal effect on bone parameters in high-resolution peripheral quantitative computed tomography: a pilot study 


\begin{abstract}
Background

Carbon-fiber-reinforced poly-ether-ether-ketone (CFR-PEEK) has superior radiolucency compared to other orthopedic implant materials, e.g. titanium or stainless steel, thus allowing metal-artifact-free postoperative monitoring by computed tomography (CT). Recently, high-resolution peripheral quantitative $C T$ (HRpQCT) proved to be a promising technique to monitor the recovery of volumetric bone mineral density (vBMD), microarchitecture and biomechanical parameters in stable conservatively treated distal radius fractures. When using HRpQCT to monitor unstable distal radius fractures that require volar distal radius plating for fixation, radiolucent CFR-PEEK plates may be a better alternative to currently used titanium plates to allow for reliable assessment. In this pilot study, we assessed the effect of a volar distal radius plate made from CFR-PEEK on bone parameters obtained from HRpQCT in comparison to two titanium plates.
\end{abstract}

\title{
Methods
}

Plates were instrumented in separate cadaveric human forearms $(n=3)$. After instrumentation and after removal of the plates duplicate HRpQCT scans were made of the region covered by the plate. HRpQCT images were visually checked for artifacts. vBMD, micro-architectural and biomechanical parameters were calculated, and compared between the uninstrumented and instrumented radii.

\section{Results}

No visible image artifacts were observed in the CFR-PEEK plate instrumented radius, and errors in bone parameters ranged from $-3.2 \%$ to $2.6 \%$. In the radii instrumented with the titanium plates, severe image artifacts were observed and errors in bone parameters ranged between $-30.2 \%$ and $67.0 \%$.

\section{Conclusions}

We recommend using CFR-PEEK plates in longitudinal in vivo studies that monitor the healing process of unstable distal radius fractures treated operatively by plating or bone graft ingrowth. 


\section{Background}

Carbon-fiber-reinforced poly-ether-ether-ketone (CFR-PEEK) is increasingly being used as a material for orthopedic implants, e.g. in spinal cages, hip prostheses, or intramedullary nails. ${ }^{1-4}$ One of the main advantages of this material is that CFR-PEEK has been found to have superior radiolucency as compared to other materials that are conventionally used for orthopedic implants, such as stainless steel or titanium. Therefore, implants made out of CFR-PEEK allow metal-artifact-free postoperative monitoring by computed tomography (CT), magnetic resonance imaging (MRI) and radiographs.'

Recently, it has been shown that the repair process of stable distal radius fractures can be monitored longitudinally in vivo using high-resolution peripheral quantitative computed tomography (HRpQCT), ${ }^{5,6}$ For clinical and research purposes, it would be of great interest to monitor the repair process of unstable fractures or the ingrowth of bone graft or bone graft substitutes after corrective osteotomy as well. Whereas stable distal radius fractures are usually treated by immobilization with a fiberglass cast, which only has a limited effect on the bone parameters obtained by $\mathrm{HRpQCT}^{7}$, volar distal radius plates (VDRPs) as internal fixation are a generally accepted treatment in case of unstable distal radius fractures or after corrective osteotomy. ${ }^{8}$ Since VDRPs are usually made from stainless steel or, more common, from titanium, they cause severe artifacts on $\mathrm{CT}$ and HRpQCT images ${ }^{9}$, including scattering, beam hardening and streak occurrence. ${ }^{10}$ Besides reduced sensitivity of $\mathrm{CT}$ measurements, this may result in an over- or underestimation of the bone density, micro-architectural and biomechanical parameters that are derived from such images. ${ }^{9}$

Besides other implants, VDRPs made of CFR-PEEK were recently developed. Although it is widely accepted that polymer devices are more compatible with CT compared to metal devices, it is unknown whether also the quantitatively obtained bone parameters obtained from HRpQCT images remain unaffected. We expect that a CFR-PEEK plate will lead to less artifacts in HRpQCT images as compared to metal plates, and thus might be a suitable alternative to the currently used titanium plates in studies monitoring the healing process of unstable fractures or ingrowth of bone graft or bone graft substitutes after corrective osteotomy.

Therefore, the aim of this study was to compare the effect of VDRPs made of titanium or made of CFR-PEEK on the occurrence of artifacts in HRPQCT images and on the volumetric bone mineral density (vBMD), micro-architectural and biomechanical parameters that are obtained from these images. 


\section{Methods}

\section{Volar distal radius plates}

Three types of commercially available VDRPs from different firms were compared in this study. Plate \#1 (Standard five-hole Volar Distale Radius Plate, Icotec AG, Switzerland) had a thickness of $2.5 \mathrm{~mm}$ and consisted of CFR-PEEK to which $0.5 \%$ tantalum fibers were added for radiopacity. Plate \#2 (VariAx ${ }^{\mathrm{TM}}$ Standard Anatomical Volar Distal Radius Plate, Strycker Corporation, USA) was $2 \mathrm{~mm}$ thick and made of titanium alloy Ti6Al4V, which contained $89 \%$ titanium, $6 \%$ aluminum and $4 \%$ vanadium. Plate\# 3 (2.4 mm Variable Angle LCP Volar Extra-Articular Distal Radius Plate, Synthes AG, Switzerland) was $2.4 \mathrm{~mm}$ thick and also made of a titanium alloy, i.e. Ti6Al7 Nb containing $86 \%$ titanium, $6 \%$ aluminum and $7 \%$ niobium.

\section{Fresh frozen forearms}

For this study three intact human forearms (right side) were isolated from three cadavers. A handwritten and signed codicil from each donor, posed when still alive and well, is kept at the Department of Anatomy and Embryology, Faculty of Health, Medicine and Life Sciences, Maastricht University, Maastricht, The Netherlands. This is required by Dutch law for the use of cadavers for scientific research and education. The forearms were thawed for eight hours at $16^{\circ} \mathrm{C}$ before surgery was started.

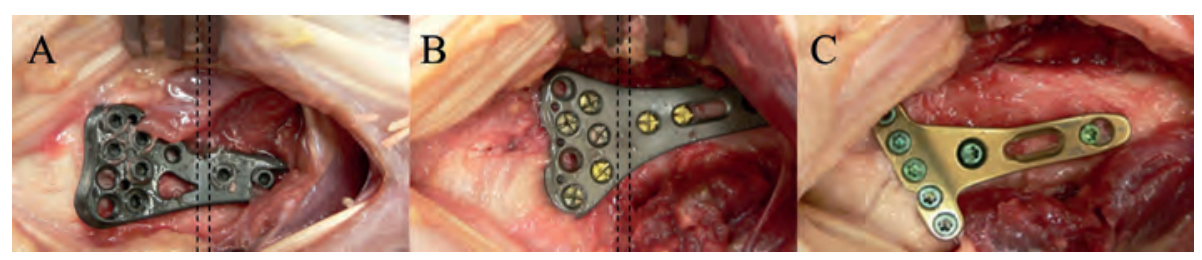

Figure 1 Each volar distal radius plate was instrumented in one cadaveric forearm by means of a standard volar approach. The CFR-PEEK plate is shown on the left. In each plate, five screws were fixed in the metaphysis and two screws were fixed in the shaft. The region of interest (between dotted lines) was set such that it was fully covered by a part of the plate without holes.

\section{Surgery}

Each plate was instrumented by an experienced orthopedic surgeon (PW) in one forearm by means of a standard volar approach and fixed to the distal radius according to the instruction manual provided by the manufacturer. During surgery, employees of the respective firms were present to support the surgeon and to make sure the proper surgical techniques were used. In all plates, five screws were fixed in the metaphysis distally from the region of interest (ROI) and two screws were fixed in the shaft proximally from the ROI (Figure 1). The screws were made of 
the same material as the respective plate. The plates were removed after HRpQCT scans had been performed.

\section{HRpQCT scanning}

Both after instrumentation and after removal of the plates, the forearms were scanned twice, with repositioning between each scan, by HRpQCT (XtremeCT, Scanco Medical AG, Switzerland) using clinical in vivo settings by the manufacturer (60 kVp effective energy, $900 \mu \mathrm{A}$ tube current, and $100 \mathrm{~ms}$ integration time). A standard stack of 110 transverse images with an isotropic voxel size of $82 \mathrm{~mm}$ was acquired, covering a region of $9 \mathrm{~mm}$ in each forearm. The ROI was set at the distal end of the radius and which was covered by a part the plate without screw holes. Also, the ROI was chosen such that it was located in between the five distal and two proximal screws to eliminate distortion by the screws (Figure 2).

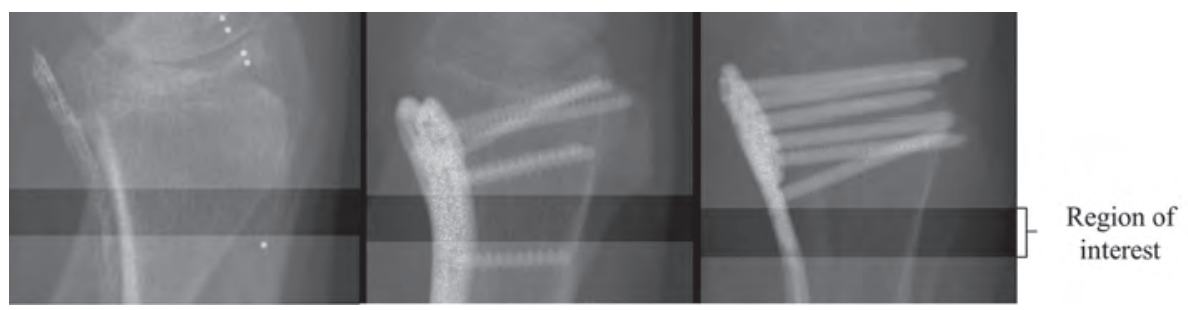

Figure 2 Lateral scout views of each instrumented radius showing the region of interest (ROI) in each radius. The $\mathrm{ROI}$ was chosen at a location where the radius was fully covered by a part of the plate without holes and that was not intersected by screws. The tantalum fibers in the CFR-PEEK plate (left) allow visualization of the plate.

\section{Bone density and micro-architecture}

The HRpQCT images within the ROI were evaluated using the standard patient evaluation protocol provided by the manufacturer and has been described earlier in detail elsewhere. ${ }^{11}$ In short, after contouring of the periosteal boundary of the radius, the total region was separated into a cortical and trabecular region. For each region, the volumetric bone mineral density (Dtot, Dcort and Dtrab, respectively) $\left[\mathrm{mgHA} / \mathrm{cm}^{3}\right]$ was assessed. The mineralized bone was extracted and a segmented image was created ${ }^{11}$ from which the following micro-architectural parameters were assessed: trabecular number (Tb.N) [ $\left.\mathrm{mm}^{-1}\right]$, thickness (Tb.Th) $[\mathrm{mm}]$ and separation (Tb.Sp) [mm]. For the cortical region, the thickness (Ct.Th) [mm] was calculated. ${ }^{11}$

\section{Micro finite element analysis}

$\mu \mathrm{FE}$ models were created directly from the segmented HRpQCT images, similar to earlier studies. ${ }^{12,13}$ In short, each voxel representing bone was converted into a 
brick element of the size, thus creating a representative $\mu \mathrm{FE}$ model of the bone's micro-architecture. Typically, these $\mu \mathrm{FE}$ models consisted of three to four million elements. Equal material properties were assigned to every element, i.e. a Young's modulus of $10 \mathrm{GPa}$ and a Poisson ratio of $0.3 .{ }^{13}$ By applying a 'high friction' compression test in the axial direction as described by Pistoia et al., the following biomechanical parameters were estimated: stiffness (Scomp) [kN/mm], which can be described as the resistance against displacement; and ultimate failure load (F.Ult) [kN], which is the load at which the fracture criterion is met, e.g. the strain in at least $2 \%$ of the volume exceeds $0.7 \% .^{12}$

\section{Statistics}

Reproducibility of the bone density, micro-architectural and biomechanical parameters was expressed using the root mean-square coefficient of variation (RMSCV\%). ${ }^{14}$ Means for the bone parameters in each instrumented and uninstrumented radius were calculated from the duplicate measurements. The errors in the bone parameters introduced by each plate were expressed as percent differences between the instrumented and uninstrumented radius, with the uninstrumented radius as $100 \%$ reference.

\section{Results}

\section{HRpQCT images}

HRpQCT images of each radius with and without plate are shown in Figure 3. The tantalum fibers that were incorporated into the CFR-PEEK plates were clearly visible, and no visible image artifacts were observed when the CFR-PEEK plate was instrumented (Figure $3 \mathrm{~A}$ ). In the radii instrumented with the titanium plates, severe image artifacts were seen (Figure $3 B$ and $3 C$ ). These image artifacts consisted of streaks and a higher intensity of the bone voxels that are closely located to the plate.

\section{Errors in bone parameters}

The reproducibility (RMS-CV) of the bone density, micro-architectural and biomechanical parameters ranged from 0.0 to $2.3 \%$ (Table 1 ).

In Table 2, the bone parameters measured per uninstrumented and instrumented distal radius are shown, together with the percent errors that were introduced by each plate. All errors in bone parameters are in comparison to the same but uninstrumented radius. For the radius instrumented with the CFR-PEEK plate, percent errors in bone parameters ranged from $-3.2 \%$ in Ct.Th to $+2.6 \%$ 


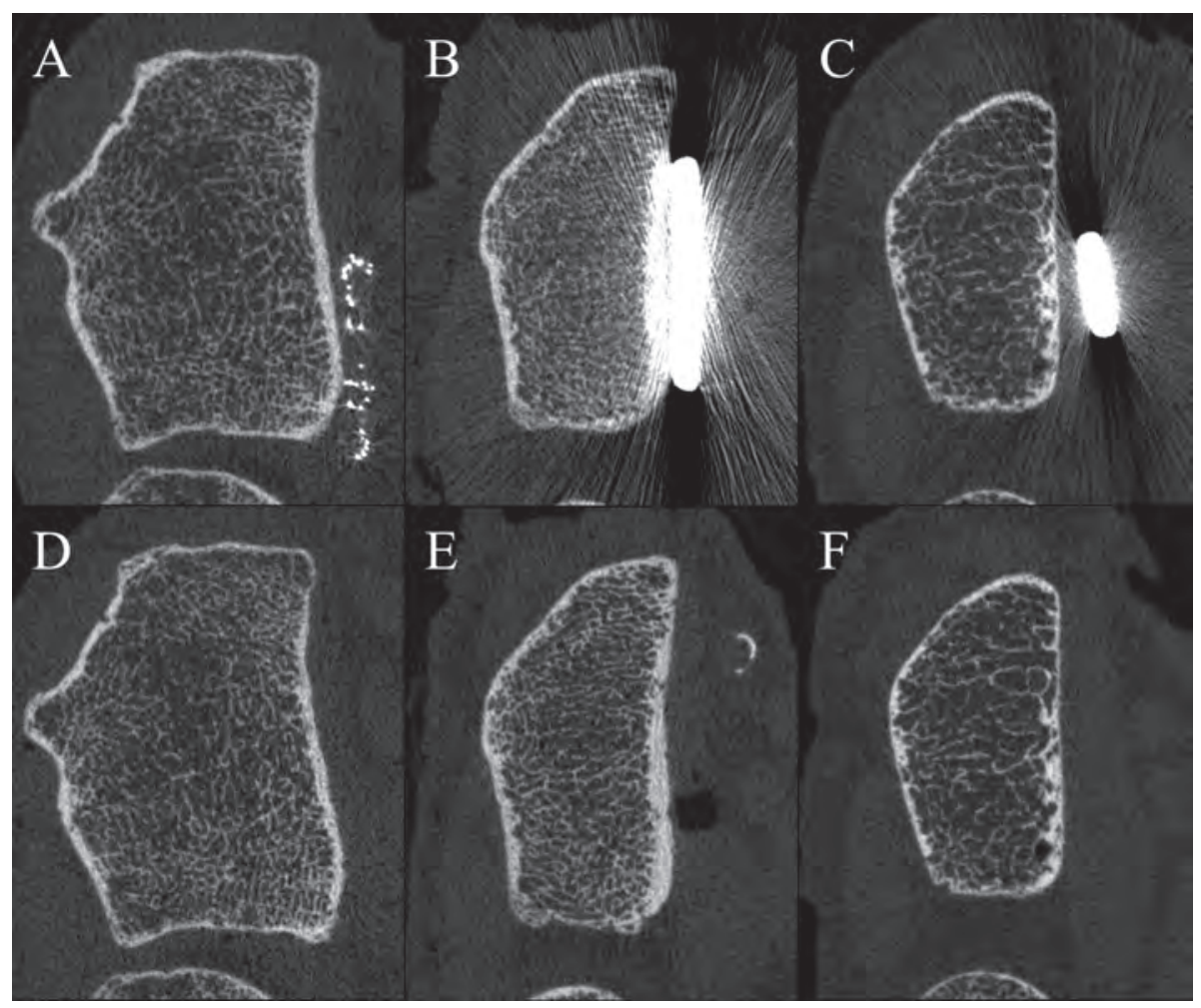

Figure 3 Representative HRpQCT slices in each radius with (top row) and without instrumented plate (bottom row). The CFR-PEEK plate with tantalum fibers (panel $3 \mathrm{~A}$ ) caused less visible image artifacts as compared to the titanium plates (panel $3 \mathrm{~B}$ and $3 \mathrm{C}$ ).

in Tb.Sp, and the smallest error was observed in Dtrab, with $-0.8 \%$. For the radii instrumented with the titanium plates, in general the percent errors were larger. For the radius instrumented with plate \#2, the percent errors in bone parameters ranged from $-43.2 \%$ in Tb.Sp to $+67.2 \%$ in Dtrab, and the smallest error was observed in Scomp, with $+7.5 \%$. For the radius instrumented with plate \#3, the largest percent errors in bone parameters were also found in Tb.Sp and Dtrab, being $-11.2 \%$ and $+30.2 \%$, respectively. The smallest error was observed in F.Ult, with $+0.1 \%$. 


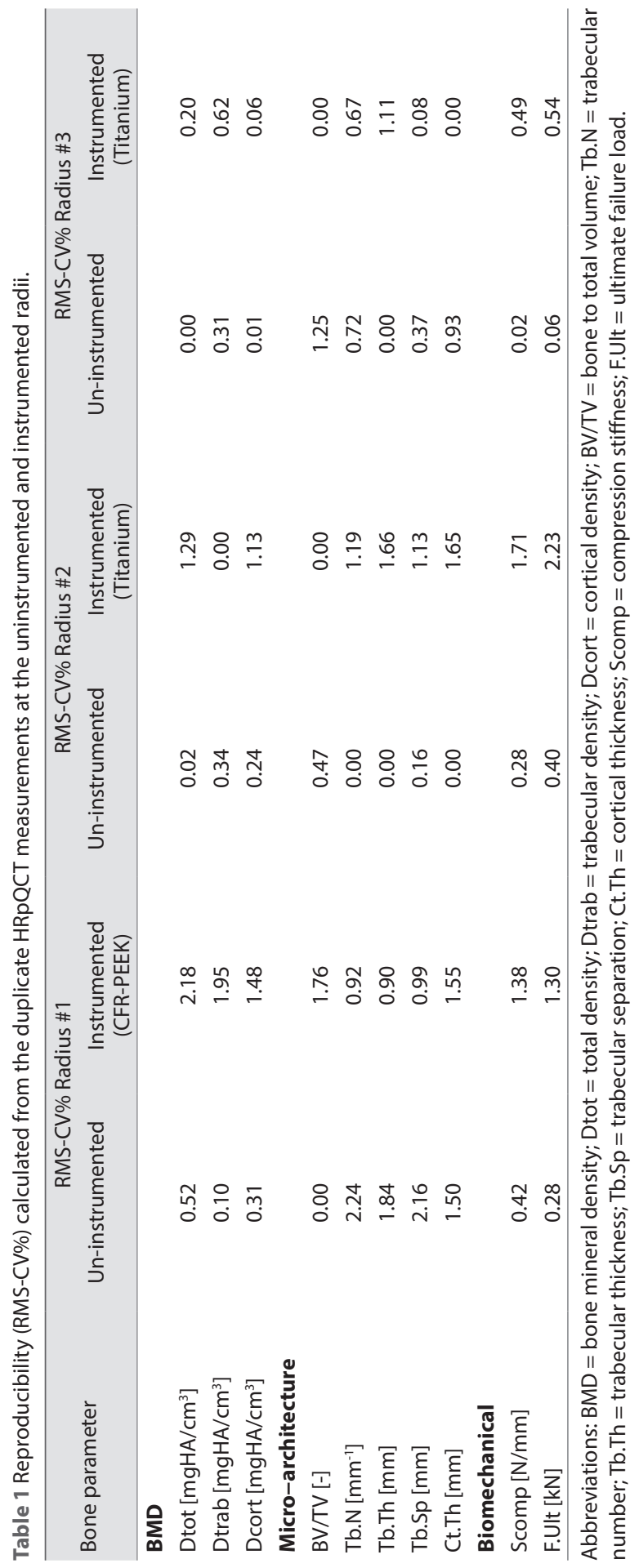




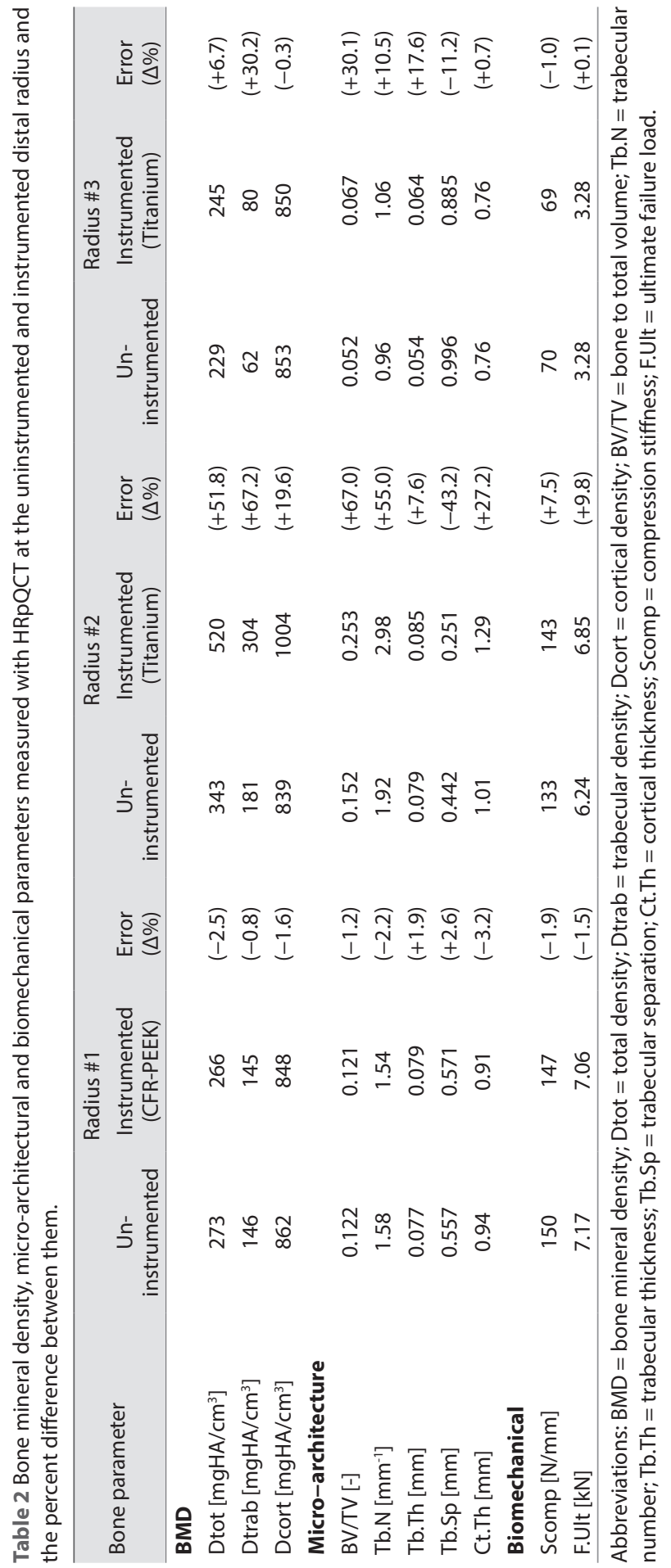




\section{Discussion}

This study tested the effect of CFR-PEEK and two titanium VDRPs on bone density, micro-architectural and biomechanical parameters at the distal radius obtained by HRpQCT in combination with $\mu$ FEA. Compared to conventional VDRPs that are made from titanium alloys, the plate made from CFR-PEEK introduced no visible image artifacts and had a minimal effect on the assessment of VBMD, microarchitectural and biomechanical parameters.

As expected, severe image artifacts were introduced by the titanium plates, which in turn led to an overestimation of the BMD, trabecular number and thickness and biomechanical properties, and an underestimation of trabecular separation at the distal radius. The errors in these bone parameters, however, differed between both titanium plates and this difference is probably related to the positioning and geometry of the plates: plate \#2 was wider, thicker, and as can be seen in Figure 3, more closely instrumented onto the radius, whereas plate \#3 was smaller and a small gap between the bone and the plate was left after instrumentation. Nevertheless, the magnitude of the errors introduced by the titanium plates is higher than the reproducibility of the HRpQCT technique. Additionally, due to differences in positioning and size of the implants it seems almost impossible to correct the bone parameters in a systematic way, which makes these titanium plates unsuitable in studies when one wants to monitor bone healing with HRpQCT.

The CFR-PEEK plate, on the other hand, introduced relatively small errors that were of the same order as the reproducibility of the HRpQCT technique. ${ }^{7,11,15}$ A plate made from CFR-PEEK would therefore be a suitable alternative to the conventional volar distal radius plates, if one wants to monitor the healing process of unstable distal radius fractures with $\mathrm{HRPQCT}$ or any other $\mathrm{X}$-ray technique.

Besides the low number of subjects, a limitation of the present study is that each plate was instrumented on a different radius. However, all results were specified relative to those of the same radius without a plate, and the density and micro-architectural parameters of the radius on which the CFR-PEEK plate was instrumented was inbetween those of the radii to which a titanium plate was attached, making it unlikely that this has affected the results in any way. Another important limitation of this pilot study is the difference in geometry between the three plates that were tested. It is likely that a thick, wide plate, such as plate \#2, introduces more severe image artifacts and hence larger errors in bone parameters, than a thin, small plate. Furthermore, we did not test VDRPs made from stainless steel. The main reason is that at present 
in the Netherlands, where the study was conducted, the commonly used plates are made from titanium alloy. However, since stainless steel causes more severe image artifacts in $\mathrm{CT}$ than titanium ${ }^{16}$, it is expected that stainless steel plates would lead to higher errors in HRpQCT-derived bone parameters as compared to titanium plates.

\section{Conclusion}

In conclusion, the results of this pilot study indicate that a volar distal radius plate made from CFR-PEEK has minimal effect on bone parameters obtained at the distal radius with $\mathrm{HRpQCT}$. We therefore recommend the use of CFR-PEEK plates instead of conventional titanium plates in studies that aim to monitor the healing process of distal radius fractures or bone graft ingrowth at the distal radius over time using HRpQCT.

\section{Declarations}

Ethics approval and consent to participate A handwritten and signed codicil from each donor, posed when still alive and well, is kept at the Department of Anatomy and Embryology, Faculty of Health, Medicine and Life Sciences, Maastricht University, Maastricht, The Netherlands. This is required by Dutch law for the use of cadavers for scientific research and education.

Competing interests B. van Rietbergen is a consultant for Scanco Medical AG. J.J. Arts is a board member of workgroup Biotechnology of the Dutch Orthopedic Association (NOV) and a board member of the Dutch Society for Biomaterials and Tissue Engineering (NBTE). P.C. Willems is a board member of the Dutch Spine Society (association of spine surgeons).

Funding This study was supported by funding of the Weijerhorst Foundation (WH2).

Authors contributions Research design: JdJ, AL, JA and PW. Acquisition, analysis or interpretation of the data: JdJ, AL, BvR, JA, PG, JvdB and PW. Drafting the manuscript: JdJ, $A L, B v R$ and PW. Revising the manuscript: JdJ, $A L, B v R, J A, P G$, JvdB and PW. All authors have read and approved the final submitted manuscript.

Acknowledgements We thank A. Gisep (Icotec AG), H. Gerritsen (Stryker) and D. Mobers (Synthes $\mathrm{GmbH}$ ) for providing the plates, screws, instrumentation kits and technical assistance. Furthermore, we thank J. Hekking and L. Huiberts from the Anatomy and Embryology department of Maastricht University for their support before and after surgery and preparation of the cadaver materials. 


\section{References}

1. Zimel, M.N., Hwang, S., Riedel, E.R. \& Healey, J.H. Carbon fiber intramedullary nails reduce artifact in postoperative advanced imaging. Skeletal Radiol 44, 1317-25 (2015).

2. Heary, R.F., Kheterpal, A., Mammis, A. \& Kumar, S. Stackable carbon fiber cages for thoracolumbar interbody fusion after corpectomy: long-term outcome analysis. Neurosurgery 68, 810-8; discussion 818-9 (2011).

3. Pace, N., Marinelli, M. \& Spurio, S. Technical and histologic analysis of a retrieved carbon fiber-reinforced poly-ether-ether-ketone composite alumina-bearing liner 28 months after implantation. J Arthroplasty 23, 151-5 (2008).

4. Nakahara, l. et al. In vivo implant fixation of carbon fiber-reinforced PEEK hip prostheses in an ovine model. J Orthop Res 31, 485-92 (2013).

5. de Jong, J.J. et al. Fracture Repair in the Distal Radius in Post-Menopausal Women: A Follow-Up Two Years Post-Fracture Using HRpQCT. J Bone Miner Res 31, 1114-22 (2016).

6. de Jong, J.J. et al. Assessment of the healing process in distal radius fractures by highresolution peripheral quantitative computed tomography. Bone 64C, 65-74 (2014).

7. de Jong, J.J. et al. Effect of a Cast on ShortTerm Reproducibility and Bone Parameters Obtained from HRpQCT Measurements at the Distal End of the Radius. J Bone Joint Surg Am 98, 356-62 (2016).

8. Hammert, W.C., Kramer, R.C., Graham, B. \& Keith, M.W. AAOS appropriate use criteria: treatment of distal radius fractures. $J A m$ Acad Orthop Surg 21, 506-9 (2013).
9. Mueller, T.L. et al. Mechanical stability in a human radius fracture treated with a novel tissue-engineered bone substitute: a noninvasive, longitudinal assessment using high-resolution $\mathrm{PQCT}$ in combination with finite element analysis. J Tissue Eng Regen Med 5, 415-20 (2011).

10. Boas, F.E. \& Fleischmann, D. Evaluation of two iterative techniques for reducing metal artifacts in computed tomography. Radiology 259, 894-902 (2011).

11. Boutroy, S., Bouxsein, M.L., Munoz, F. \& Delmas, P.D. In vivo assessment of trabecular bone micro-architecture by high-resolution peripheral quantitative computed tomography. J Clin Endocrinol Metab 90, 6508-15 (2005).

12. Pistoia, W. et al. Estimation of distal radius failure load with micro-finite element analysis models based on three-dimensional peripheral quantitative computed tomography images. Bone 30, 842-8 (2002).

13. Pistoia, W. et al. Image-based micro-finiteelement modeling for improved distal radius strength diagnosis: moving from bench to bedside. J Clin Densitom 7, 153-60 (2004).

14. Gluer, C.C. et al. Accurate assessment of precision errors: how to measure the reproducibility of bone densitometry techniques. Osteoporos Int 5, 262-70 (1995).

15. MacNeil, J.A. \& Boyd, S.K. Improved reproducibility of high-resolution peripheral quantitative computed tomography for measurement of bone quality. Med Eng Phys 30, 792-9 (2008).

16. Knott, P.T. et al. A comparison of magnetic and radiographic imaging artifact after using three types of metal rods: stainless steel, titanium, and vitallium. Spine J 10, 78994 (2010). 



$$
\frac{b}{x}
$$




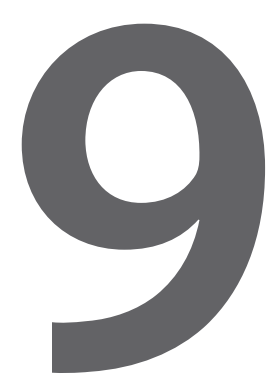

General discussion 
In this thesis, for the first time the healing process of distal radius fractures treated by cast immobilization has been studied in vivo by non-invasive HRpQCT imaging. After describing the effect of plaster-of-Paris and fiberglass casts on HRpQCT measurements (Chapter 2), the healing process itself was studied using HRpQCT. During an initial short-term follow-up period of 12 weeks post-fracture, changes in bone density, micro-architecture and biomechanical parameters that occurred during the healing process were analyzed extensively (Chapter 3), including their relation to the clinical outcome as assessed by the PRWE questionnaire (Chapter 4). In addition, a long-term follow-up at two years post-fracture was conducted to compare the restored fracture side to the contra-lateral side (Chapter 5) as well as to study the longitudinal changes at the non-fractured side (Chapter 6). Last, we addressed two aspects related to the feasibility of HRpQCT to study the healing process of unstable distal radius fractures, i.e. the feasibility of 3D registration of the fragments (Chapter 7), and the influence of a radiolucent volar distal radius plate on HRpQCT scans and the bone parameters (Chapter 8).

In this final chapter, the main findings and limitations of the previous chapters are discussed, and general conclusions and recommendations as well as directions for future research are provided.

\section{Main findings and limitations}

\section{Issues and artifacts related to HRpQCT imaging of distal radius fractures}

Cast immobilization is the recommended treatment for stable distal radius fractures, while unstable distal radius plates are preferably treated surgically by plating. ${ }^{1}$ Casts as well as distal radius plates may cause image artifacts in HRpQCT and therefore affect vBMD, micro-architectural and biomechanical parameters that are obtained from these images.

As described in Chapter 2, wearing a forearm cast introduced systematic errors in the bone parameters. With errors ranging from $-2.6 \%$ in trabecular separation to $-9.8 \%$ in cortical thickness, a plaster-of-Paris cast considerably influences the measurement results, as a result of the interference caused by the presence of high attenuating calcium sulfate. ${ }^{2}$ The errors caused by the fiberglass cast, on the other hand, were marginal with errors between $-0.6 \%$ and $-1.6 \%$ in trabecular separation and cortical thickness, respectively, and, although statistically significant, these errors may not be clinically relevant given the fact that changes observed during fracture-healing are generally in the range of $10 \%$ to $20 \%$. In studies that aim to monitor the healing process of stable distal radius fractures, we therefore 
recommend immobilization with a fiberglass cast and to wear this cast during all HRpQCT measurements.

Volar distal radius plates, used for fixation in unstable fractures, of CFR-PEEK and titanium were compared in Chapter 8. Compared to the currently used titanium volar distal radius plates, a volar distal radius plate made from radiolucent CFRPEEK has minimal effect on bone parameters obtained from HRpQCT with errors in bone parameters between $-0.8 \%$ and $-3.2 \%$ in trabecular vBMD and cortical thickness, respectively. Furthermore, the CFR-PEEK plate introduced no visible artifacts, thus allowing for reliable assessment of vBMD and micro-architecture during the healing process of unstable distal radius fractures that are treated with such a plate. Because also the biocompatibility, material characteristics, and mechanical durability of CFR-PEEK orthopaedic implants are promising ${ }^{3}$, we therefore recommend the use of CFR-PEEK plates instead of conventional titanium plates in studies that aim to monitor the healing process of unstable distal radius fractures or bone graft ingrowth at the distal radius over time using HRpQCT.

Another important image artifact that needs to be addressed is the partialvolume effect (PVE). Although not limited to HRpQCT imaging of fractures, PVEs are inherent to imaging of bone micro-architecture with HRpQCT and depend on the resolution. As a result of the PVE, small bone structures, such as thin cortices or trabeculae that typically span one to three voxels at the XtremeCT's resolution, appear blurred in the HRpQCT images. After segmentation of the HRpQCT images, these thin structures may be averaged away in the segmented 3D models and, therefore, leading to an underestimation of trabecular number and overestimation of cortical thickness. Especially in fracture healing, where old trabecular remnants are resorbed and new trabeculae and cortical bone are formed, this is unwanted effect. Probably with the recent introduction of the second generation HRpQCT scanner (XtremeCT2; Scanco Medical A.G. Brütisellen, Switzerland), the PVE is less present thanks to the increased resolution of the XtremeCT2, enabling direct assessment of trabecular and cortical micro-architecture. ${ }^{4,5}$

One more important source of potential error is patient motion, which severely reduces the reproducibility of the bone micro-architecture and, hence, biomechanical parameters ${ }^{6}$ and results in approximately $30 \%$ of the radius scans being of insufficient quality for analysis. ${ }^{7}$ In our fracture healing cohort (Chapter 3 and 5), $17 \%$ of the HRpQCT scans at the fracture side and $28 \%$ at the contra-lateral side was of insufficient quality, even with implementation of one repeat scan in our protocol when the first scan was of insufficient quality. In our reproducibility study (Chapter 2), the majority (88\%) of the scans with insufficient quality were scans made without a cast. Although not statistically proven, these results indicate that patient motion is at least partially reduced when scanning a subjects' forearm in a 
cast. This is not surprising, as the cast is custom-made (for fracture stabilization and healing) and therefore allows a secure fixation of the patient's forearm. In research using HRpQCT, one might therefore consider to scan the study subjects' forearm using a cast-like fixation device as this may reduce the number of required subjects due to the lower number of HRpQCT measurements that are unusable because of motion artifacts.

\section{Distal radius fracture healing assessed by HRpQCT}

The changes that were observed in the HRpQCT images of the healing fractures as well as in the vBMD, micro-architectural and biomechanical parameters during the first twelve weeks of healing were striking (Chapter 3 ). As expected, total vBMD showed a peak at six to eight weeks post-fracture, which is consistent with previous studies using conventional QCT. ${ }^{8}$ Interestingly, however, separate evaluation of the $\mathrm{VBMD}$ in the cortical and trabecular region revealed that opposite changes occurred in these regions. Whereas trabecular vBMD showed a peak at six to eight weeks post-fracture and returned to normal at twelve weeks post-fracture, cortical vBMD decreased throughout the twelve-week follow-up period. Furthermore, at the micro-architectural level, there was a reduction in trabecular number of $15 \%$ but an increase in trabecular thickness of 30\%, indicating intense bone remodeling, with resorption of the trabecular structure together with new bone formation at the surface of remaining or newly formed trabeculae. At the same time, the bone biomechanical parameters increased by more than $30 \%$.

The results of the two-year follow-up HRpQCT measurements (Chapter 5) showed that most of these changes still progressed over time. Compared to the contra-lateral side, BMD became similar at two years post-fracture. However, in the period from six and twelve weeks to two years post-fracture, the cortical thickness at fracture side increased, while concurrently a decrease in trabecular number and an increase in trabecular separation occurred whereas the trabecular thickness remained high. These results might suggest that the cortical region is restored at the expense of the trabecular region. Whether this is the result of a shift in load-bearing from the trabecular to the cortical region due to different use of the fractured wrist, or is caused by other processes, remains to be studied.

Simultaneous to the changes in the cortical and trabecular region, bone stiffness at fracture side showed a major increase of more than $100 \%$ in the period from six weeks to two years post-fracture. This result could indicate that by the time the cast is removed, bone stiffness is far from recovered. However, this is not necessarily problematic as patients are able to use their wrist in normal daily activities, which most likely do not require the bone stiffness to be fully restored. 


\section{Changes at the non-fractured distal radius}

In contrast to the fracture side, no changes in the bone parameters were observed at the contra-lateral side during the first twelve weeks after fracture (Chapter 3 and Chapter 6). However, a two-year decrease of almost $4 \%$ in cortical vBMD was found, but not in total vBMD. Additionally, a reduction in bone strength and stiffness had occurred of more than $5 \%$. The finding that we did not observe a change in total VBMD is consistent with current literature that assessed aBMD by DXA at the contra-lateral radius after a Colles' fracture. ${ }^{9}$ However, the two-year decreases in cortical density and bone strength and stiffness were higher than expected, as other longitudinal studies reported a two-year percent change in cortical vBMD and bone strength between $-1 \%$ to $-2 \%$ in the control group, which consisted of post-menopausal women who were mostly osteopenic. ${ }^{10-12}$ More surprisingly, accelerated cortical bone loss at the contra-lateral side occurs in patients with a distal radius fracture, even in patients compliant with adequate anti-resorptive drug treatment.

Since the fracture side became stronger in the same period, one might wonder if the fracture region is restored at the expense of bone at other sites, even at the contra-lateral side. If that would be the case, then a decrease in spine and hip BMD would also be expected. Unfortunately, we did not longitudinally monitor VBMD or bone micro-architecture at other sites than the distal radius, so we can only speculate here.

\section{D image registration}

For accurate longitudinal analysis of bone parameters, it is important that the same region is analyzed. In unstable fractures, fragments may move relative to each other, and this displacement needs to be corrected in order to analyze the same region. We showed that superposition of subvolumes, such as fracture fragments, in a follow-up image onto a baseline image is feasible using 3D rigid image registration, and that this leads to better alignment of these fragments compared to an approach where the whole fractured region in a follow-up image is registered on a baseline image (Chapter 7).

Although more research is required to optimize the registration procedure, the current result is promising for reliable monitoring of VBMD and microarchitecture in unstable distal radius fractures, and additionally also the analysis of bone resorption and formation based on longitudinal HRpQCT images of healing fractures, for example to study the metabolic effect of anti-osteoporosis medication on fracture healing. 


\section{Limitations}

The work presented in this thesis has several limitations. First, due to the exploratory nature of the studies, the numbers of subjects is rather small and due to motion artifacts, several HRpQCT measurements were excluded from statistical analyses thus leading to missing values. In addition, we had multiple HRpQCT parameters to test, which are in part related to each other. In order to deal with missing values, we applied a linear mixed-effect model instead of a repeated measurement design. By using the Bonferroni correction in the linear mixed-effect model we corrected for the multiple comparisons between the visits in Chapter 3, but we did not correct for the fact that we tested eleven different bone parameters because of the exploratory study design. In the linear regression models in Chapter 4, no correction for multiple comparisons was applied while a correction such as the Benjamini-Hochberg procedure ${ }^{13}$ might have been appropriate. Nonetheless, in retrospect, a post-hoc analysis using the Benjamini-Hochberg correction with a false discovery rate of 0.20 revealed that the conclusions did not change after correction for multiple comparisons, i.e. the associations between early change in trabecular VBMD, separation and torsional stiffness with PRWE score at twelve weeks post-fracture remained significant. In addition, this post-hoc analysis showed that apart from our original findings also the early change in total VBMD was significantly associated with the PRWE score at twelve weeks post-fracture.

Second, in the distal radius fracture studies, the proximal edge of the lunate bone instead of the distal radius plateau was used as reference point. However, the joint space width between the lunate bone and the radius might not be constant over time, thus increasing the positioning error and therefore lowering the reproducibility of the bone parameters. Although the variation is joint space width over time is unknown, a recent reproducibility exercise in reference line placement showed that a variation in positioning of $0.38 \mathrm{~mm}$ leads to extra imprecision errors (RMS-CV\%) of $2.4 \%$ in vBMD and $5.8 \%$ in cortical thickness, whereas microarchitecture and failure load remain unaffected. ${ }^{14}$ Hence, as these precision errors are considerably smaller than the changes found in the bone parameters during healing, it is unlikely that variations in joint space width affected the results.

Third, no correction was applied for the measurements that were performed with the forearm in a plaster-of-Paris cast at the first visit, despite of the errors that this type of plaster causes. However, taken into account that more than half of the patients (56\%) was wearing a fiberglass cast and not a plaster-of-Paris cast at the first visit, together with the fact that the required corrections for errors of $2 \%$ to $8 \%$ are much smaller than the observed changes during fracture healing, it is likely that correcting for the plaster-of-Paris cast would lead to similar conclusions. 
Fourth, in the fracture to non-fracture side comparisons we did not take dominance into account as we assumed that there were no left-right differences in bone parameters. This seems legitimate, as Hildebrandt et al. found no significant differences in BMD and micro-architecture measured by HRpQCT at the distal radius between the dominant and non-dominant forearm, and failure load was only 3\% higher at the dominant side.15 However, in our study most fractures occurred at the non-dominant side ( $n=9,74 \%$ ), thus correcting for hand dominance would have strengthened the conclusion regarding the stiffness parameters in the sideto-side comparison in Chapter 5.

Fifth, there are limitations with respect to the use of the $\mu \mathrm{FEA}$ in this thesis. First of all, only mineralized tissue was taken into account in the FE models, whereas also other supportive tissue, like low-mineralized woven bone, is present at the fracture site. The actual biomechanical competence of the fracture region might therefore be underestimated by the $\mu \mathrm{FEA}$, especially during the early stages of fracture healing, i.e. up to six to eight weeks post-fracture. Second, the $\mu F E A$ is only validated for unfractured bone. Unfortunately, it will be hard or even impossible to address this limitation adequately, i.e. experimentally measure bone strength in healing fractures in humans and compare it to the bone strength calculated from the $\mu \mathrm{FEA}$, since the validation would raise some serious ethical concerns.

Last, the study was set up such that bone changes during the early phases of the fracture healing process, i.e. up to twelve weeks post-fracture, could be studied during frequently scheduled study visits. The remodeling phase, however, continues for a longer period. No HRpQCT scans were planned beyond the study period of twelve weeks, since METC approval for additional scanning procedures could only be acquired after demonstrating that it was feasible to assess fracture healing with HRpQCT based on the results of the first twelve-week study period. Once METC approval for an extra visit was obtained, time had passed up to two years after initial fracture. As a result, it remains unclear at what time point between twelve weeks and two years the remodeling phase as assessed by HRpQCT parameters actually normalized. In future longitudinal HRpQCT studies on fracture healing, we therefore advise to schedule follow-up visits after the initial twelveweek period more regularly, e.g. at least every six months post-fracture.

\section{Clinical relevance}

This thesis showed that we are now able to evaluate vBMD, micro-architecture and bone stiffness during the healing process of a stable distal radius fracture. Furthermore, we found significant associations with $\mathrm{R}^{2}$ ranging from $31 \%$ to $37 \%$ between the early changes in HRPQCT derived bone parameters and the PRWE score, representing clinical outcome such as pain and function, at twelve weeks post- 
fracture (Chapter 4). These associations are better compared to previously reported baseline patient- and injury related factors ( $R^{2}$ between $16 \%$ and $25 \%$ ). ${ }^{16,17}$ Based on these findings, one could argue that it might be useful to investigate whether it is feasible to shorten or prolong a cast-immobilization period of an individual, based on the estimated stiffness of the fractured region. In the context of fracture healing, however, the methods that we used to evaluate vBMD, micro-architecture and biomechanical parameters from the HRpQCT images still have issues that need to be further addressed. For instance, the micro-architectural parameters in the early phases of the healing process need to be interpreted with caution due to the presence of low-mineralized bone, and it has not been proven that (changes in) these parameters are a meaningful representation of bone consolidation during healing. Nevertheless, we believe that, in contrast to plain radiographs and clinical examination, we now have a quantitative method available for the assessment of micro-architecture and biomechanical competence that we can further develop and validate to analyze in detail stable and more complex distal radius fractures with dislocation and after surgery, as well as to study the effect of metabolic bone disorders and drugs on mineralization, modeling and remodeling of trabecular and cortical bone during the fracture healing process.

\section{Conclusion and recommendations}

In conclusion, this thesis has resulted in the following conclusions and recommendations:

1. In contrast to a plaster-of-Paris cast, a fiberglass cast has no relevant effect on the reproducibility of HRPQCT scans and the calculation of the bone parameters from these scans. It is therefore recommended to use a fiberglass cast in studies monitoring the healing process of stable distal radius fractures using HRpQCT.

2. After opposite changes in bone mineral density of the cortical and trabecular region during the first twelve weeks of distal radius fracture healing, bone density becomes comparable to the bone density at the contra-lateral side at two years post-fracture. Additionally, trabecular micro-architecture seemed to be decreased, yet bone stiffness at fracture side was restored beyond the contra-lateral bone stiffness after two years.

3. Early changes in HRpQCT derived bone parameters can predict pain and functional outcome better than patient- and injury related factors. The relation between the $\mathrm{HRpQCT}$ derived bone parameters and the longterm clinical outcome has yet to be investigated. 
4. Despite adequate anti-resorptive treatment, a significant, higher than expected, two-year decrease in cortical density and biomechanical parameters at the contra-lateral side was observed in our fracture healing cohort.

5. Monitoring the healing process of unstable distal radius fractures with HRpQCT is feasible when radiolucent distal volar radius plates are used to stabilize the fracture, and in addition 3D image registration is applied to accurately overlay the different fracture fragments.

6. $\mathrm{HRpQCT}$, in combination with $\mu \mathrm{FEA}$, is a promising technique in fracture healing related research since it provides information at the microscale on bone densitometric, micro-architectural and biomechanical changes. Future research on fracture healing, e.g. in studies on the effect of medication on the healing process, should therefore consider using HRpQCT as their preferred imaging tool.

\section{Future perspectives}

The studies described in this thesis showed that the healing process of stable distal radius fractures can be studied in detail using HRpQCT and $\mu \mathrm{FEA}$. It is however needed that our findings will be reproduced in larger series. In addition, HRpQCT in combination with $\mu F E A$ is a promising tool for future research on fracture healing.

One of the future opportunities would be to use HRpQCT and $\mu$ FEA to study the effect of medication on fracture healing in more detail. For instance, it is known that $60 \%$ to $70 \%$ of the patients with a recent fracture are vitamin D deficient ${ }^{18}$, and that vitamin $\mathrm{D}$ supplementation has a positive effect on fracture healing in animals ${ }^{19}$. Since clinical studies that address the clinical effects of vitamin $D$ deficiency or supplementation on fracture healing are scarce and remain inconclusive ${ }^{20}$, it would be very interesting to study the effect of immediate vitamin D supplementation on the healing process of distal radius fractures by HRpQCT. In addition, postmenopausal women often receive anti-osteoporotic drugs as they suffer from osteoporosis. These anti-osteoporosis drugs, e.g. bisphosphonate, strontium renalate, parathyroid hormone, anti-sclerostin, denosumab, have anticatabolic and/or anabolic mechanisms of action, and may affect fracture healing by suppressing bone resorption and formation during the different phases of the healing process. ${ }^{21,22}$ Although it is reported that anti-osteoporosis drugs do not have a negative impact on fracture healing in clinical practice ${ }^{21-23}$, we propose to study whether these drugs influence the fracture healing process on a microarchitectural level with HRpQCT. 
Second, our studies, however small $(n=3)$, suggest that 3D registration allows precise monitoring of specific regions over time and that the use of radiolucent plates allows reliable assessment of unstable distal radius fractures. It would therefore be of great interest to monitor the healing process of surgically treated fractures, and also the evaluation of ingrowth of bone graft may benefit from the abilities of HRpQCT.

Third, whereas this thesis focused on the healing process of distal radius fractures, HRpQCT can also be applied to study the fracture healing process at other common fracture sites at the peripheral parts of the body. For example, the treatment of scaphoid fractures is challenging, and failure can result in avascular necrosis (up to $40 \%$ ), nonunion (up to $21 \%$ ) of the fracture and subsequently early osteoarthritis (up to $32 \%$ ). ${ }^{24-27}$ Similar to the distal radius, the scaphoid bone is mainly composed of trabecular bone, thus using HRpQCT in studies on scaphoid fracture healing treatment might also shed a new light on the causes of poor outcome in scaphoid fractures.

From a clinical perspective, our results showed that early changes in trabecular vBMD and separation were associated with the short-term (twelve-week) clinical outcome of the fractured distal radius. Since the trabecular region is mostly comprised in such fractures, it might well be that also the long-term outcome is related to (changes in) these parameters. This could be relevant for clinicians, as it may be of added value for optimizing fracture healing treatment.

HRpQCT is currently only available as a research tool. Because of its limited availability world-wide and considerable cost, it should be investigated if FEA based on QCT scans of lower resolution ${ }^{28}$, can provide comparable information regarding changes in $\mathrm{VBMD}$ and biomechanical parameters during the fracture healing process.

The recent development of the second generation HRpQCT scanners, i.e. XtremeCT2, on the other hand, allows studying $\mathrm{VBMD}$, bone micro-architecture and biomechanical parameters using a higher resolution of $61 \mathrm{~mm} .^{4,5}$ Whether more reliable results with regard to the healing process of distal radius fractures can be obtained with any of these techniques is unknown, thus future research should focus on a direct comparison between the three techniques in assessing the healing process of distal radius fractures. 


\section{References}

1. The Association of Surgeons of the Netherlands. Guideline Distal Radius Fractures: Diagnosis and Treatment (Dutch) (Utrecht, 2010).

2. Wytch, R., Ashcroft, G.P., McKenzie, G., Wardlaw, D. \& Ledingham, W.M. Radiographic assessment of splinting bandages. Injury 22, 41-4 (1991).

3. Li, C.S., Vannabouathong, C., Sprague, S. \& Bhandari, M. The Use of CarbonFiber-Reinforced (CFR) PEEK Material in Orthopedic Implants: A Systematic Review. Clinical Medicine Insights. Arthritis and Musculoskeletal Disorders 8, 33-45 (2015).

4. Manske, S.L., Zhu, Y., Sandino, C. \& Boyd, S.K. Human trabecular bone micro-architecture can be assessed independently of density with second generation HRpQCT. Bone 79, 213-21 (2015)

5. Agarwal, S. et al. In vivo assessment of bone structure and estimated bone strength by first- and second-generation HRpQCT. Osteoporos Int 27, 2955-66 (2016).

6. Pialat, J.B., Burghardt, A.J., Sode, M., Link, T.M. \& Majumdar, S. Visual grading of motion induced image degradation in high-resolution peripheral computed tomography: impact of image quality on measures of bone density and microarchitecture. Bone 50, 111-8 (2012).

7. Geusens, P. et al. High-resolution in vivo imaging of bone and joints: a window to micro-architecture. Nat Rev Rheumatol 10, 304-13 (2014).

8. Grigoryan, M. et al. Quantitative and qualitative assessment of closed fracture healing using computed tomography and conventional radiography. Acad Radiol 10, 1267-73 (2003).

9. Ingle, B.M., Hay, S.M., Bottjer, H.M. \& Eastell, R. Changes in bone mass and bone turnover following distal forearm fracture. Osteoporos Int 10, 399-407 (1999).

10. Burghardt, A.J. et al. A longitudinal HRpQCT study of alendronate treatment in postmenopausal women with low bone density: Relations among density, cortical and trabecular micro-architecture, biomechanics, and bone turnover. J Bone Miner Res 25, 2558-71 (2010).

11. Zhu, T.Y. et al. Cortical thinning and progressive cortical porosity in female patients with systemic lupus erythematosus on long-term glucocorticoids: a 2-year casecontrol study. Osteoporos Int (2015).
12. Cheung, A.M. et al. Effects of odanacatib on the radius and tibia of postmenopausal women: improvements in bone geometry, micro-architecture, and estimated bone strength. J Bone Miner Res 29, 1786-94 (2014).

13. Benjamini, Y. \& Hochberg, Y. Controlling the False Discovery Rate: A Practical and Powerful Approach to Multiple Testing. Journal of the Royal Statistical Society. Series B (Methodological) 57, 289-300 (1995).

14. Bonaretti, S. et al. Operator variability In Scan Positioning is a Major Component of HRpQCT Precision Error and is Reduced by Standardized Training. Osteoporos Int (In Press) (2016).

15. Hildebrandt, E.M., Manske, S.L., Hanley, D.A. \& Boyd, S.K. Bilateral Asymmetry of Radius and Tibia Bone Macroarchitecture and Microarchitecture: A High-Resolution Peripheral Quantitative Computed Tomography Study. J Clin Densitom 19, 250-4 (2016).

16. MacDermid, J.C., Donner, A., Richards, R.S \& Roth, J.H. Patient versus injury factors as predictors of pain and disability six months after a distal radius fracture. J Clin Epidemiol 55, 849-54 (2002).

17. Grewal, R., MacDermid, J.C., Pope, J. \& Chesworth, B.M. Baseline predictors of pain and disability one year following extraarticular distal radius fractures. $\operatorname{Hand}(N Y) \mathbf{2}$ 104-11 (2007).

18. Gorter, E.A., Krijnen, P. \& Schipper, I.B. Vitamin $D$ deficiency in adult fracture patients: prevalence and risk factors. Eur J Trauma Emerg Surg 42, 369-78 (2016).

19. Fu, L., Tang, T., Miao, Y., Hao, Y. \& Dai, K. Effect of 1,25-dihydroxy vitamin D3 on fracture healing and bone remodeling in ovariectomized rat femora. Bone 44, 893-8 (2009).

20. Gorter, E.A., Hamdy, N.A., Appelman-Dijkstra, N.M. \& Schipper, I.B. The role of vitamin D in human fracture healing: a systematic review of the literature. Bone 64, 288-97 (2014).

21. Hegde, V., Jo, J.E., Andreopoulou, P. \& Lane, J.M. Effect of osteoporosis medications on fracture healing. Osteoporos Int 27, 861-71 (2016)

22. Larsson, S. \& Fazzalari, N.L. Anti-osteoporosis therapy and fracture healing. Arch Orthop Trauma Surg 134, 291-7 (2014). 
23. Li, Y.T., Cai, H.F. \& Zhang, Z.L. Timing of the initiation of bisphosphonates after surgery for fracture healing: a systematic review and meta-analysis of randomized controlled trials. Osteoporos Int 26, 431-41 (2015).

24. Divelbiss, B.J. \& Adams, B.D. Electrical and ultrasound stimulation for scaphoid fractures. Hand Clin 17, 697-701, x-xi (2001).

25. Rajagopalan, B.M., Squire, D.S. \& Samuels, L.O. Results of Herbert-screw fixation with bone-grafting for the treatment of nonunion of the scaphoid. J Bone Joint Surg Am 81, 4852 (1999).
26. Raudasoja, L., Rawlins, M., Kallio, P. \& Vasenius, J. Conservative treatment of scaphoid fractures: a follow up study. Ann Chir Gynaecol 88, 289-93 (1999).

27. Tiel-van Buul, M.M. et al. Radiography and scintigraphy of suspected scaphoid fracture. A long-term study in 160 patients. J Bone Joint Surg Br 75, 61-5 (1993).

28. Cody, D.D. et al. Femoral strength is better predicted by finite element models than QCT and DXA. J Biomech 32, 1013-20 (1999). 


\section{Addendum}

Valorization

Summary

Samenvatting

Dankwoord

Curriculum Vitae

Publications 


\section{Valorization}

During my research on fracture healing at the distal radius, I have only seen a fraction of all patients that visited the emergency department after they sustained a fracture. Yet, even in this small group of patients I noticed that patients are mostly looking forward to the moment of cast removal. Since I have never fractured a bone in my body, I can only imagine how annoying it must be not being able to scratch where it itches or not being able to wash away the sweat (and smell) on hot summer days. Would it therefore not be great if the time of wearing a cast is kept to a minimum for each individual patient? Besides these benefits on an individual level, reduction of cast immobilization time could also benefit the society by lowering the costs that are indirectly related to distal radius fractures, such as loss of productivity or days of sick-leave.

Since the main purpose of a cast is to provide the necessary stability until the fractured bone has regained sufficient bone strength, the time to cast removal can be reduced by simply speeding up bone strength recovery. However, in order to assess bone strength recovery in the individual patient, a quantitative non-invasive method is required. In this thesis, we mainly focused on exploring the use of a new imaging method because plain radiographs that are currently used to study the fracture healing process lack detailed quantitative information regarding the consolidation of the micro-architecture and neither do they provide quantitative information on the restoration of bone strength.

In 2005, high-resolution peripheral quantitative computed tomography (HRpQCT) was introduced. With a resolution of $82 \mathrm{~mm}$ it has the capability to assess the bone's micro-architecture. In addition, 3D models can be created from these highresolution images which in turn can be used in micro finite element analysis in order to estimate bone strength. Whereas most research on bone quality mainly used these techniques to study bone quality during aging, under healthy and diseased conditions, and following medication, we explored, for the first time ever, the potential of HRpQCT in combination with newly developed imaging analysis techniques to study the fracture healing process. We showed that we can reliably monitor the fracture healing process of distal radius fractures during the first twelve weeks using $\mathrm{HRpQCT}$ in terms of bone density, micro-architecture and bone strength parameters, and that changes in these parameters were associated with clinical outcome, such as pain and disability, at the end of this twelve-week period. Also, we developed algorithms that could visualize and quantify the impressive amounts of bone modeling and remodeling during the fracture healing process in high detail. 
In order to achieve this, a combined effort of several departments in the different stages of the study was required: starting with providing patient information to eligible patients at the emergency department, to inclusion and short-term follow-up by the orthopaedic surgeon, and finally the long-term follow-up by the endocrinologist. In other words, we have gained valuable experience in how to set up a framework for conducting fracture healing related research in a clinical setting.

Based on the framework and concepts we developed while studying the feasibility of HRpQCT to monitor the fracture healing process for more than 5 years, we believe that Maastricht University Medical Center is currently one of the expert centers in the world for clinical research on fracture healing. That said, it is now feasible to collaborate with pharmaceutical companies in clinical trials to study the effect of their drugs on the fracture healing process. Both safety aspects and probable positive effects regarding fracture healing can now be studied with the algorithms we developed. It would be interesting to invite pharmaceutical companies to participate in studies with existing drugs. In this way, only a minor investment of their side is required. In return, they can potentially use existing drugs for new purposes. Since this does not involve the high costs that are associated with development of new drugs, the risk-benefit ratio for the pharmaceutical companies is rather small. For example, these kind of studies could already be performed with 30 patients ( 10 controls, 20 on treatment) during six visits that are scheduled within the first year after fracture.

Also for non-pharmaceutical companies working together with the HRpQCT group at the Maastricht University Medical Center might be interesting. For instance, companies that develop medical devices that aim to improve fracture healing, can collaborate with us to study the effect of their device in patients with a fracture in more detail than ever before. These results could then be used to optimize the design, leading to a more effective medical device.

Such collaborations with industry are also beneficial for Maastricht University and its research schools. First of all, with these funds a new PhD-student can be appointed. Furthermore, pharmaceutical and medical device companies are often operating world-wide. Collaborating with such an international partner would add to the international allure that Maastricht University envisions.

In the end, however, these developments, i.e. new drugs or medical devices that aim to improve the fracture healing outcome, should result in shorter healing times, better functional recovery and less pain in patients with a fracture. 


\section{Summary}

One of the most common types of fractures is a fracture of the wrist, i.e. the distal radius. These fractures are usually treated by cast immobilization or surgically by internal or external plating. Unfortunately, the clinical outcome of the fracture healing process is not always good and many factors that contribute to a poor outcome are still unknown.

In clinical practice, fracture healing is evaluated using plain radiographs in combination with physical examination. However, these assessments do not provide detailed information regarding consolidation of the bone microarchitecture or the restoration of bone strength. With the introduction of highresolution peripheral quantitative computed tomography (HRpQCT) imaging, the micro-architecture of bone can now be studied in patients. In addition, based on three-dimensional HRpQCT images, bone strength can be estimated using micro finite element analysis ( $\mu \mathrm{FEA})$. With regards to this thesis, we hypothesized that the use of HRpQCT and $\mu \mathrm{FEA}$ attributes to a better understanding of distal radius fracture healing.

Although HRpQCT and $\mu \mathrm{FEA}$ have been used to study bone quality during aging, with different disorders or after medication, they have never been applied in a clinical setting to study the repair of fractures. The aim of this thesis, therefore, was to explore the application of HRpQCT in combination with $\mu \mathrm{FEA}$ to longitudinally study the healing process of distal radius fractures.

Casts and internal plates are commonly used to treat stable and unstable distal radius fractures, respectively. They may introduce image artifacts in HRpQCT and, therefore, affect the bone parameters that are derived from such images. As shown in Chapter 2, a fiberglass cast introduces a significant but not clinical relevant error in the bone parameters of up to $-1.6 \%$, whereas a cast made from plaster of Paris leads to larger errors up to $-9.8 \%$. In case of plating (Chapter 8), volar distal radius plates made from carbon-fiber-reinforced polyetheretherketone (CFRPEEK) introduced no visible image artifacts and errors where smaller than $3.2 \%$. In contrast, the titanium plates caused severe image artifacts and led to errors in bone parameters that ranged from $-30.2 \%$ to $67.0 \%$.

Based on these findings, we therefore recommend the use of a fiberglass cast for stable fractures and a CFR-PEEK plate for unstable fractures to monitor the fracture healing process of distal radius fractures with HRPQCT.

In Chapter 3, the healing process of distal radius fractures was described in terms of HRpQCT derived bone density, micro-architectural and biomechanical parameters 
for the first twelve weeks after fracture. Additionally, in Chapter 4, the correlation between these HRpQCT derived bone parameters and the clinical outcome as assessed by the PRWE questionnaire was studied. In eighteen post-menopausal women with a stable distal radius fracture treated by cast immobilization, we found an increase compared to baseline in total and trabecular VBMD of $11 \%$ and $20 \%$ (both $p<0.01$ ), respectively, at six weeks post-fracture. The occurrence of intertrabecular bone formation was indicated by a 23\% and 31\% $(p<0.01)$ increase in trabecular thickness at six and twelve weeks post-fracture, respectively, and blurring of the intertrabecular area. Bone stiffness at twelve weeks post-fracture was increased by $31 \%$ compared to baseline $(p<0.01)$. In addition, a lower PRWE score (representing less pain and better function) at twelve weeks post-fracture was significantly related to an early increase in trabecular vBMD $(\beta-0.96, p=0.022$, $\left.R^{2}=0.37\right)$ and torsional stiffness $\left(\beta-0.14, p=0.037, R^{2}=0.31\right)$, and an early decrease in trabecular separation $\left(\beta 209, p=0.045, R^{2}=0.33\right.$ ).

This exploratory study demonstrated that it is feasible to monitor the healing process of stable distal radius with HRpQCT during the first twelve weeks after fracture, and that HRpQCT can provide valuable information regarding the shortterm clinical outcome in terms of pain and disability.

Since the healing process is not completed at twelve weeks post-fracture, a followup visit at two years post-fracture was envisaged in order to study the long-term changes in VBMD, micro-architectural parameters and biomechanical competence at the fractured side. In addition, the fracture side was compared to the contra-lateral side. The results of this study are described in Chapter 5. In the fourteen eligible patients that participated in the two-year visit, we found significant changes in all bone parameters, except in total vBMD, trabecular thickness and cortical perimeter when comparing the measurements at six and twelve weeks with those at two years post-fracture. At two years post-fracture, cortical and trabecular thickness, and torsional and bending stiffness were significantly higher at the fractured side $(21 \%, 55 \%, 31 \%$ and $29 \%$, respectively, $\mathrm{p}<0.05)$ compared to the contra-lateral side, whereas vBMD was not significantly different between both sides. From these results, we concluded that by the time the cast is removed, i.e. at six weeks postfracture, the fracture healing process is still ongoing and large changes occur in vBMD, micro-architecture and biomechanical parameters thereafter. Compared with the contra-lateral side at two years post-fracture, the fractured side had thicker cortices and trabeculae, whereas BMD was similar. Ultimately, this resulted in a restoration of bone stiffness that ended up being significantly higher at the fracture side than at the contra-lateral side. 
Although no changes in $\mathrm{VBMD}$ are expected at the contra-lateral distal radius following a distal radius fracture, it is unknown if also bone micro-architecture and strength at the contra-lateral distal radius are unaffected after a distal radius fracture. Therefore, in Chapter 6, the bone parameters at the contra-lateral side were studied using HRPQCT and $\mu$ FEA measurements at baseline, twelve weeks and two years post-fracture. Compared to baseline, we found a significant decrease in cortical vBMD $(-3.9 \%, p<0.001)$, failure load $(-5.6 \%, p=0.007)$, and stiffness in compression $(-5.1 \%, p=0.014)$ and bending $(-6.0 \%, p=0.029)$ at two years post-fracture. These results were similar between patients with or without adequate anti-osteoporosis drug treatment. Since these changes were larger than previously reported changes due to aging, we conclude that the healing process of stable distal radius fractures in post-menopausal women may be associated with accelerated cortical bone loss and concomitant reduction of bone strength but not with micro-architectural changes at the contra-lateral distal radius, despite adequate anti-resorptive treatment.

In Chapter 7, a method for overlaying two consecutive HRpQCT scans from patients with a healing distal radius fracture was introduced. In contrast to the standard registration method in which the whole bone volumes are used to overlay the HRpQCT images, this method relies on registration of individual fragments. After testing the effect of fragment size and amount of displacement using unfractured distal radius HRpQCT scans, the method was applied in two stable and one unstable fracture(s). We found that fragments as small as $300 \mathrm{~mm}^{3}$ that were displaced up to $0.82 \mathrm{~mm}$ in-plane or up to $1.64 \mathrm{~mm}$ in the longitudinal directional could be realigned correctly. For the fractured cases, most improvement in alignment was found in the unstable fracture. This result is promising for additional analysis of bone formation and resorption in $\mathrm{HRpQCT}$ studies on fracture healing.

Finally, in Chapter 9, the main findings and limitations of the aforementioned studies are discussed. Furthermore, general conclusions and recommendations as well as directions for future are presented. 



\section{Samenvatting}

Een van de meest voorkomende fracturen is een polsfractuur, meer specifiek een breuk van de distale radius (spaakbeen). In het geval van een stabiele radiusfractuur bestaat de behandeling uit gipsimmobilisatie, terwijl bij een instabiele fractuur meestal een chirurgische ingreep nodig is om de fragmenten te stabiliseren door middel van een metalen of kunststofpolsplaatje. Helaas is de klinische uitkomst van het genezingsproces niet altijd goed en zijn niet alle factoren die bijdragen aan een slechte uitkomst bekend.

In de dagelijkse klinische praktijk wordt de vordering van het fractuurgenezingsproces vastgesteld aan de hand van röntgenfoto's in combinatie met lichamelijk onderzoek door de behandelend arts. Echter, deze methodes geven géén gedetailleerd beeld van de mate waarin de fijne botstructuren (botbalkjes) vastgegroeid zijn of van het herstel van de botsterkte. Sinds de introductie van hoge resolutie perifere kwantitatieve computer tomografie (CT) in 2005 is het mogelijk om de botstructuur van de pols tot op microniveau te bestuderen. Daarnaast kunnen de gegevens van deze driedimensionale hoge resolutie CT scans als basis dienen voor eindige elementen analyse (EEA), waarmee de sterkte van het bot berekend kan worden. Omdat met hoge resolutie CT in combinatie met EEA een gedetailleerder beeld verkregen kan worden van de botkwaliteit, is de hypothese die getoetst wordt in dit proefschrift dat het gebruik hiervan kan leiden tot een beter inzicht in het genezingsproces van distale radius fracturen.

Ondanks dat hoge resolutie CT en EEA al een aantal jaren gebruikt worden om te bestuderen hoe de botkwaliteit verandert bij het ouder worden, bij bepaalde ziektebeelden of als gevolg van medicatie, is hoge resolutie CT nog niet eerder in de kliniek ingezet om het genezingsproces van fracturen te bestuderen. Het doel van dit proefschrift is om het genezingsproces van distale radius fracturen te bestuderen met behulp van hoge resolutie CT in combinatie met EEA.

De aanbevolen behandeling van stabiele distale radius fracturen bestaat uit het dragen van een gipsverband gedurende 3 tot 5 weken. Het gebruik van gips zou echter kunnen leiden tot verstoringen in hoge resolutie CT scans, en daarmee de uitkomsten van de botparameters nadelig kunnen beïnvloeden. In hoofdstuk 2 is het effect van twee soorten gips (fiberglass en pleister gips) op hoge resolutie CT metingen vergeleken. Hieruit bleek dat zowel fiberglass als pleister gips tot significante afwijkingen leidden in de botparameters. Echter, de afwijkingen in de botparameters ten gevolge van fiberglass gips waren maximaal $-1.6 \%$ ten opzichte van metingen zonder gips en daarmee niet klinisch relevant, terwijl de afwijkingen als gevolg van pleister gips opliepen tot $-9.8 \%$. Op basis van deze 
resultaten adviseren we om in toekomstige studies naar het genezingsproces van distale radius fracturen met hoge resolutie CT gebruik te maken van fiberglass gips.

In hoofdstuk $\mathbf{3}$ is het genezingsproces van distale radius fracturen voor het eerst bestudeerd met hoge resolutie CT. Hierbij is gekeken naar de veranderingen in botdichtheid, -structuur en sterkte gedurende de eerste twaalf weken na de breuk. In achttien postmenopauzale vrouwen met een stabiele distale radius fractuur, die behandeld werd met gips, vonden we zes weken na de breuk een significant hogere totale en trabeculaire botdichtheid (respectievelijk $11 \%$ en $20 \%$ ) in vergelijking met één week na de fractuur. Ook de dikte van botbalkjes was op zes en twaalf weken na de breuk significant hoger (31\%). Daarnaast lieten de hoge resolutie CT scans mineralisatie zien tussen de botbalkjes, wat duidde op de aanmaak van bot tussen de botbalkjes in plaats van op de botbalkjes. Ten opzichte van één week na de fractuur was de botsterkte twaalf weken na de breuk significant toegenomen met $31 \%$.

Vervolgens is, in hoofdstuk 4, gekeken of de PRWE score (een klinische uitkomst score op basis van pijn en functie) twaalf weken na de breuk geassocieerd is met vroege veranderingen (in de periode tussen één en drie weken na de breuk) in de botparameters die berekend kunnen worden op basis van de hoge resolutie CT scans. In dit onderzoek werd een significante relatie gevonden tussen een betere PRWE score op twaalf weken na de breuk en grotere vroege veranderingen in het bot, namelijk een toename in trabeculaire botdichtheid en torsiestijfheid en afname in de onderlinge afstand tussen de botbalkjes.

In deze exploratieve studies hebben we aangetoond dat het haalbaar is om de eerste twaalf weken van het genezingsproces van stabiele distale radius fracturen te volgen met hoge resolutie CT, en dat veranderingen in botdichtheid, -structuur en -sterkte, die plaatsvinden in de eerste drie weken na de breuk, waardevolle informatie kunnen geven met betrekking tot de pijn en functie op twaalf weken na de breuk.

Omdat het fractuurgenezingsproces twaalf weken na de breuk nog niet voltooid is en op dat moment dus nog geen eindpunt van het genezingsproces vastgesteld kan worden, is een extra hoge resolutie CT scan uitgevoerd twee jaar na de breuk. Hierbij zijn de botdichtheid, -structuur en sterkte aan zowel de fractuur zijde als aan de (niet gebroken) contra-laterale zijde gemeten en met elkaar vergeleken. De resultaten daarvan zijn beschreven in hoofdstuk $\mathbf{5}$. Voor de tweejaars meting waren veertien van de achttien patiënten uit hoofdstuk 3 en 4 beschikbaar en bij al deze patiënten was de breuk volledig hersteld. Na twee jaar was in vrijwel alle botparameters een significant verschil meetbaar ten opzichte van de metingen zes 
en twaalf weken na de breuk, waarbij met toenames van respectievelijk $186 \%$ en $97 \%$ in de botsterkte parameters de grootste veranderingen gemeten werden. Uit de vergelijking tussen de fractuur- en contra-laterale zijde op twee jaar na de breuk bleek dat de dikte van de cortex en de botbalkjes alsmede de botsterkte significant hoger waren aan de fractuurzijde dan aan de contra-laterale zijde, terwijl de botdichtheid gelijk was. Hieruit kunnen we concluderen dat op het moment dat het gips verwijderd wordt (ongeveer zes weken na de breuk), het fractuurhelingsproces nog niet voltooid is en dat er daarna nog grote veranderingen plaatsvinden in de botdichtheid, -structuur en -sterkte. In vergelijking met de (niet gebroken) contralaterale zijde, heeft de fractuurzijde na twee jaar een dikkere cortex en dikkere botbalkjes met als gevolg een hogere botsterkte dan aan de niet gebroken zijde.

$\mathrm{Na}$ een polsbreuk wordt er op basis van eerder onderzoek geen verandering in botdichtheid aan de contra-laterale distale radius verwacht. Omdat in deze onderzoeken alleen gebruik is gemaakt van botdichtheidsmetingen is onbekend of ook de botstructuur en botsterkte aan de contra-laterale zijde onveranderd blijven na een distale radius fractuur. In hoofdstuk 6 zijn daarom zijn de botdichtheid, botstructuur, en botsterkte aan de contra-laterale pols gemeten met hoge resolutie CT en EEA op de meetmomenten één week, twaalf weken en twee jaar na de breuk. In de periode van één tot twaalf weken na de breuk werden geen veranderingen gevonden in de botparameters. Echter, twee jaar na de breuk waren de botdichtheid van de cortex en de botsterkte van de contra-laterale zijde significant lager ten opzichte van één week na de breuk (respectievelijk -3.9\% en $-5.5 \%$ ). Deze afnames werden gevonden bij zowel patiënten met als zonder botafbraakremmende medicatie. Als we deze afnames vergelijken met eerder gepubliceerde gegevens van vrouwen zonder polsbreuk in de literatuur, dan blijkt dat de in onze groep gevonden afnames groter zijn dan normaal gesproken verwacht mag worden gedurende twee jaar ouder worden. Hieruit concluderen we dat in postmenopauzale vrouwen met een stabiele distale radius fractuur een verhoogde corticale botafbraak plaatsvindt, met tegelijkertijd een afname in botsterkte, ondanks behandeling met botafbraakremmende medicatie.

Een nieuwe methode om twee opeenvolgende hoge resolutie CT beelden van genezende distale radius fracturen over elkaar heen te leggen (registreren), wordt beschreven in hoofdstuk 7. In tegenstelling tot de standaard registratie methode, waarbij gehele botvolumes worden gebruikt om de beelden over elkaar heen te leggen, is de nieuwe methode gebaseerd op de registratie van individuele fractuurfragmenten. Eerst is het effect van fragmentgrootte en mate van verplaatsing getest op hoge resolutie $C T$ beelden van niet gebroken bot, 
en vervolgens is de methode toegepast op stabiele en instabiele fracturen. Uit deze tests bleek dat fragmenten zo klein als $300 \mathrm{~mm}^{3}$ met een vooraf opgelegde verplaatsing tot $0.82 \mathrm{~mm}$ in zijwaartse richting en tot $1.64 \mathrm{~mm}$ in de lengterichting correct geregistreerd konden worden. In het geval van fracturen was de meeste verbetering in overlap te zien in de instabiele fractuur wanneer de registratie op basis van individuele fragmenten in plaats van de standaard registratie methode toegepast werd. De resultaten van deze pilot studie zijn veelbelovend voor het bestuderen van instabiele distale radius fracturen of voor het bestuderen van botaanmaak en - afbraak tijdens de fractuurgenezing, en geven aanleiding tot een verdere uitwerking van de methode en validatie ervan zodat deze daadwerkelijk toegepast kan worden.

Waar stabiele distale radius fracturen behandeld worden met gips, bestaat de behandeling van instabiele distale radius fracturen vaak uit een chirurgische ingreep waarbij een polsplaatje geplaatst wordt om de fragmenten te stabiliseren. Dergelijke polsplaatjes zijn gemaakt van titanium, wat kan leiden tot verstoringen op CT scans en dus ook op de hoge resolutie CT scans waardoor de botdichtheid, -structuur en -sterkte niet accuraat bepaald kunnen worden. Een mogelijke oplossing hiervoor is het gebruik van een polsplaatje gemaakt van carbon, een materiaal waarvan bekend is dat dit zeer weinig verstoring geeft op CT scans. In hoofdstuk 8 wordt een pilot studie beschreven waarin het effect van een carbon polsplaatje op de hoge resolutie CT scans en de botparameters is vergeleken met veelgebruikte polsplaatjes van titanium. Dit is gedaan door de polsplaatjes te instrumenteren in drie menselijke kadaverarmen en hoge resolutie CT scans van de polsen te maken met en zonder polsplaatjes. De resultaten toonden aan dat een polsplaatje van carbon niet leidt tot zichtbare verstoringen in de hoge resolutie CT scans en dat de invloed op de botparameters minimaal is. Dit in tegenstelling tot de polsplaatjes van titanium, waarbij de botparameters tot $67 \%$ afweken. Op basis van de resultaten van deze pilot studie adviseren we het gebruik van carbon polsplaatjes in studies waarbij het genezingsproces van instabiele distale radius fracturen gevolgd wordt met hoge resolutie CT.

Tot slot, in hoofdstuk 9, worden de hoofdbevindingen en beperkingen van de afzonderlijke studies besproken. Tevens wordt een overzicht gegeven van de algemene conclusies en worden aanbevelingen voor toekomstig onderzoek beschreven. De voornaamste conclusie is dat hoge resolutie $C T$, in combinatie met eindige elementen analyse, een veelbelovende techniek is om het genezingsproces van distale radius fracturen te bestuderen omdat het belangrijke informatie verschaft met betrekking tot (veranderingen in) de botdichtheid, 
-structuur en -sterkte tijdens de fractuurgenezing. Het wordt dan ook aanbevolen om in toekomstig onderzoek naar het fractuurgenezingsproces, bijvoorbeeld in studies naar het effect van medicatie op de fractuurgenezing, hoge resolutie CT in te zetten als voornaamste imaging techniek. 



\section{Dankwoord}

Bij het uitvoeren van de beschreven onderzoeken zijn veel mensen betrokken geweest, en allemaal hebben zij op één of andere manier hun steentje bijgedragen aan de totstandkoming van dit boekje. Daarvoor wil ik iedereen hartelijk bedanken, waarvan een aantal personen in het bijzonder.

In de eerste plaats bedank ik mijn promotieteam voor hun expertise, de soms pittige discussies maar bovenal de prettige samenwerking, die overigens al startte tijdens mijn afstudeerstage. Ik denk dat we elkaar, met clinici enerzijds en technici anderzijds, goed hebben kunnen aanvullen, wat uiteindelijk heeft geleid tot dit mooie resultaat waar we met zijn allen trots op mogen zijn.

Prof. dr. Geusens, beste Piet, uw aanstekelijke enthousiasme en creatieve manier van denken maakten dat het een waar genoegen was om met u onderzoek te doen. Ik heb genoten van onze discussies over "wat méten we nu?". Uw perspectief op het fractuurhelingsproces was fascinerend en ik hoop dat u me vergeeft dat niet alle klinische aspecten even logisch zijn voor een niet-clinicus als ik; bedankt voor uw geduld. Daarnaast heeft u me geleerd dat het soms nodig is om diplomatiek te zijn, een belangrijke eigenschap om verder te komen in het leven! Merci!

Prof. dr. van den Bergh, beste Joop, bedankt voor de dagelijkse begeleiding en niet te vergeten het mogelijk maken van mijn promotie. Je positieve houding en je out-of-the-box denken waren voor mij en het onderzoek erg waardevol. Dankzij jou heb ik kunnen samenwerken met veel andere onderzoeksgroepen, waar ik veel van heb geleerd en wat me veel heeft opgeleverd. Tot slot, je betrokkenheid, begrip en vertrouwen, ook in moeilijkere perioden, maakten dat ik altijd op mijn eigen manier kon werken. Dank je wel!

Dr. Willems, beste Paul, als enige orthopeed was je van het begin af aan betrokken bij de fractuurhelingstudie. Jouw expertise met betrekking tot de klinische praktijk was onmisbaar voor een goed verloop van de studies en bovenal een fijne aanvulling op de endocrinologische inzichten. Bovendien stond je deur altijd open, wat het samenwerken met jou erg prettig maakte. Bedankt daarvoor!

Dr. ir.van Rietbergen, beste Bert, bijzonder bedankt dat ik altijd bij je kon aankloppen als ik vragen had, en dat al sinds mijn eerste orthopaedie project tijdens mijn master. Je nuchtere kijk op dingen en rustige manier van werken waardeer ik zeer. 
Ook inhoudelijk heb ik veel van je mogen leren. Het was voor mij dan ook een groot plezier met je samen te mogen werken!

De leden van de leescommissie prof. dr. van Rhijn, prof. dr. Ito, prof. dr. van Lenthe en dr. Weijers bedank ik voor de moeite en tijd die zij hebben genomen voor het lezen en beoordelen van mijn proefschrift.

Daarnaast wil ik alle coauteurs hartelijk bedanken voor hun samenwerking. Een aantal van hen wil ik graag bij naam wil noemen:

Prof. dr. Müller, dear Ralph, it was a pleasure to be part of your research group for three months, thank you. You and your group are dedicated, and working with all of you was inspiring. I enjoyed the nice atmosphere, the raclette evening and, last but not least, the fabulous ski-event.

Dr. Arts, beste Chris, bedankt voor je inzichten tijdens onze meetings en je nuttige feedback op mijn manuscripten. Als hoofd van het trialbureau heb je bovendien zorg gedragen voor een belangrijk logistiek deel van de studie. Succes met je nieuwe project!

Drs. Bours, beste Sandrine, ontzettend bedankt voor jouw inzet tijdens mijn afstudeerstage en met name (maar zeker niet alleen) de eerste periode van mijn promotietraject. Je hebt mijn promotie een kick-start gegeven en mede daardoor is het me gelukt deze vlot af te ronden. Binnenkort is het ook jouw beurt. Heel veel succes met de laatste loodjes!

Dr. Christen, dear Patrick, thank you for your enthusiasm and your hospitality during my time at ETH. I learned a lot from you. Not only on image analysis, but you also taught me the principles of a good Swiss fondue.

Dr. Meyer, dear Ursina, it was a pleasure to work with you. Your patience and thoroughness have led to a great publication. Thank you also for your hospitality in Zurich and I wish you good luck with your own research using HRpQCT.

Dr. van Geel, beste Tineke, ook jij bedankt voor het op weg helpen van mijn promotie. Je kritische houding en behulpzaamheid heb ik zeer gewaardeerd. 
Dr. Poeze, beste Martijn, bedankt voor je heldere inzichten met betrekking tot het fractuurgenezingsproces en je doordachte input tijdens de meetings. Ik heb er zeker wat aan gehad.

Dr. Keszei, dear András, thank you for your help with the statistics and for your patience while explaining them, even when you already had left Maastricht and moved to Aachen.

Dr. Lataster, beste Arno, bedankt voor de uitstekende ondersteuning door de afdeling Anatomie en Embryologie bij de distale radius studies. Het was voor jou, Johan en Leon nooit een probleem om materiaal gereed te maken of te bewaren, of mee te denken over de studieopzet. Dank jullie wel.

Dr. Menheere, beste Paul, bedankt voor je inzichten over de botmarkers tijdens fractuurgenezing. Het analyseren van de bloedsamples was voor u en Nancy altijd een kleine moeite. Dank jullie wel voor de samenwerking.

Ook was het onderzoek nooit geslaagd zonder inzet van de volgende personen:

Prof. dr. Boonen, beste Annelies, bedankt dat ik welkom was op uw afdeling. Ondanks dat onze onderzoeksgebieden enigszins verschillen, toonde $u$ altijd oprechte belangstelling in mijn onderzoek en heb ik mogen merken dat u zich ook persoonlijk betrokken voelt bij de mensen op uw afdeling, waarvoor dank.

Beste Yvonne, bedankt voor je geduld en altijd vrolijke lach op het secretariaat. Erg fijn dat het je vaak lukte nog een gaatje te vinden in de overvolle agenda van 'de twee heren'.

Het team van De Maastricht Studie, bedankt voor jullie flexibele houding tegenover de fractuurhelingstudie, waardoor de metingen aan 'mijn' patiënten altijd konden doorgaan.

Trialbureau Orthopaedie, beste Liesbeth en Margareth, erg bedankt voor het includeren en inplannen van de patiënten, het meedenken over en het uitvoeren van een belangrijk deel van de praktische kant van onderzoek. Na vier jaar zit de fractuurhelingstudie er voor jullie op: wat een opluchting!

Beste Sasha en Ed, fijn dat jullie de afgelopen jaren aan de fractuurhelingstudie dachten als op de spoedeisende hulp een patiënt met een polsfractuur binnenkwam. Bedankt voor jullie inzet en oplettendheid. 
En natuurlijk wil ik ook alle patiënten en deelnemers zelf bedanken die aan de studies hebben deelgenomen. Zonder jullie geen data, en zonder data geen resultaten. Bedankt voor jullie tijd en interesse in ons onderzoek, we hebben er veel van geleerd!

Van alle collega's zie je je medepromovendi uiteindelijk het meest. Ik wil jullie dan ook bedanken voor de leuke tijd in de hoek op de vijfde van UNS40. We hebben hoogte- en dieptepunten gedeeld, iets wat niet altijd vanzelfsprekend kan met collega's. Mayke, de tweede ingenieur op de afdeling en een welkome afwisseling. Bedankt dat je er voor me was (en vaak nog bent)! Frans, Michiel, Simon en Casper, bedankt voor de gezonde rivaliteit tijdens het wielrennen en/ of de mannenavonden! Carmen, Ivette en later Ellis, met jullie als kamergenoten was het in ieder geval nooit saai, én nooit stil. Lieke en Andrea, ik zal nooit kunnen tippen aan jullie sportiviteit, gelukkig zitten we qua humor meer op hetzelfde niveau. Jullie allemaal, samen met Antje, José, Lisanne en Caroline (hoewel geen promovenda meer), bedank ik voor alle gezellige uitjes, borrels, diners, stap-, spelletjes- en FIFA-avonden. Niet alleen in Maastricht, maar ook zeker tijdens vakanties en congresbezoek. Heel veel succes met jullie eigen onderzoek!

Michiel en Frans, jullie noem ik nog een keer want wat mij betreft waren jullie het ideale duo om mij als paranimfen bij te staan bij mijn verdediging. Niet alleen omdat we samen onderzoek hebben gedaan, maar vooral omdat we veel hebben gelachen met elkaar. Michiel, je typische Brabantse gezelligheid hebben tot de nodige afleiding en memorabele momenten geleid. Tof dat je altijd in was voor een drankje of een stapje. Frans, je gevatte opmerkingen in combinatie met je perfectionisme en to-dolijstjes zorgden ervoor dat ik altijd op je kon rekenen, zowel bij de scanner als daarbuiten. Allebei ontzettend bedankt!

Niet direct betrokken bij mijn onderzoek en juist daarom ook een bedankje waard, zijn alle vrienden en familie die interesse hebben getoond in mijn onderzoek óf juist voor de nodige afleiding gezorgd hebben. Een speciale plek krijgen Maike (met Bart en Mats) en Ward (met Mara). Als zus en broer laten jullie me altijd welkom voelen, ik ben blij dat we elkaar hebben!

Tot slot, papa en mama. Ik weet dat jullie het niet nodig vinden om in dit dankwoord genoemd te worden. Jullie hebben echter het meest bijgedragen aan wat ik tot nu toe heb bereikt, dus: dank je wel voor álles! 



\section{Curriculum Vitae}

Joost Johannes Antoin de Jong was born on 21 December 1984 in Hulst, The Netherlands. He completed secondary school at the Reynaert College in Hulst in 2003. In 2004, he started studying Mechanical Engineering at Fontys University of Applied Sciences in Eindhoven. After graduating for his bachelor's degree in 2007, he started his master Medical Engineering at Eindhoven University of Technology. During his master he completed an internship at University of São Paulo in Brazil and fulfilled his clinical graduation project on fracture healing studied by high-resolution peripheral quantitative computed tomography (HRpQCT) at the department of Orthopaedics of Maastricht University Medical Center.

After graduating for his master's degree in 2012, he got the opportunity to continue his research on fracture healing and HRpQCT at Maastricht University under supervision of prof. dr. P. Geusens, prof. dr. J. van den Bergh, dr. P. Willems and dr. ir. B. van Rietbergen. During his PhD period, he performed a three-month internship at the faculty of Biomedical Engineering of ETH in Zürich under supervision of prof. dr. R. Müller and dr. P. Christen. The current thesis presents the results of the work done during his $\mathrm{PhD}$ studies.

Currently, in order to increase his expertise in medical imaging, Joost is exploring the use of magnetic resonance imaging in neurologic disorders as a postdoctoral researcher under the supervision of prof. dr. W. Backes at the department of Radiology of Maastricht University Medical Center. 


\section{Publications}

\section{This thesis}

- de Jong JJ, Willems PC, Arts JJ, Bours SG, Brink PR, van Geel TA, Poeze M, Geusens $P P$, van Rietbergen B, van den Bergh JP. Assessment of the healing process in distal radius fractures by high-resolution peripheral quantitative computed tomography. Bone 64C, 65-74 (2014)

- Meyer U, de Jong JJ, Bours SG, Keszei AP, Arts JJ, Brink PR, Menheere P, van Geel TA, van Rietbergen B, van den Bergh JP, Geusens PP, Willems PC. Early changes in bone density, micro-architecture, bone resorption, and inflammation predict the clinical outcome 12 weeks after conservatively treated distal radius fractures: an exploratory study. J Bone Miner Res 29, 2065-73 (2014)

- de Jong JJ, Heyer FL, Arts JJ, Poeze M, Keszei AP, Willems PC, van Rietbergen B, Geusens PP, van den Bergh JP. Fracture Repair in the Distal Radius in Post-Menopausal Women: A Follow-Up Two Years Post-Fracture Using HRpQCT. $J$ Bone Miner Res 31, 1114-22 (2016)

- de Jong JJ, Arts JJ, Meyer U, Willems PC, Geusens PP, van den Bergh JP, van Rietbergen B. Effect of a Cast on Short-Term Reproducibility and Bone Parameters Obtained from HRpQCT Measurements at the Distal End of the Radius.J Bone Joint Surg Am 98, 356-62 (2016)

- JJ de Jong, JJ Arts, SP Bours, PC Willems, JP Bons, PP Menheere, B van Rietbergen, PP Geusens, JP van den Bergh Contra-Lateral Bone Loss at the Distal Radius in Postmenopausal Women After a Distal Radius Fracture: A Two-Year Follow-Up HRpQCT Study. Submitted

- de Jong JJ, Lataster A, van Rietbergen B, Arts JJ, Geusens PP, van den Bergh JP, Willems PC. Distal Volar Radius Plates from Carbon Fiber PEEK do not Affect Assessment of Bone Density, Micro-architectural and Biomechanical parameters by High Resolution Peripheral Quantitative Computed Tomography. Submitted

- JJ de Jong, P Christen, R Chapurlat, PP Geusens, JP van den Bergh, R Müller, $B$ van Rietbergen Feasibility of Rigid 3D Image Registration of High-Resolution Peripheral Quantitative Computed Tomography Images of Healing Distal Radius Fractures. Submitted 


\section{Other publications}

- Scharmga A, Peters M, van den Bergh JP, de Jong JJ, Loeffen D, van Rietbergen B, van Tubergen A, Weijers R, Geusens PP. Visual Detection of Cortical Breaks in Hand Joints: Reliability and Validity of High-Resolution peripheral Quantitative CT compared to MicroCT. BMC Musculoskelet Disord 11, 271 (2016)

- Barnabe C, Toepfer D, Marotte H, Hauge E, Scharmga A, Kocijan R, Kraus S, Boutroy S, Keller K, de Jong J, Williams J, Stok KS, Finzel S. Definition for Rheumatoid Arthritis Erosions Imaged with High Resolution Peripheral Quantitative Computed Tomography and Inter-Reader Reliability for Detection and Measurement. J Rheumatol 43, 1935-40 (2016)

- Jacobs E, Saralidze K, Roth AK, de Jong JJ, van den Bergh JP, Lataster A, Brans BT, Knetsch ML, Djordjevic I, Willems PC, Koole LH. Synthesis and characterization of a new vertebroplasty cement based on gold-containing PMMA microspheres. Biomaterials 82, 60-70 (2016)

- Geusens P, Chapurlat R, Schett G, Ghasem-Zadeh A, Seeman E, de Jong J, van den Bergh J. High-resolution in vivo imaging of bone and joints: a window to microarchitecture. Nat Rev Rheumatol 10, 304-13 (2014)

- Bogie R, Roth AK, Faber S, de Jong JJA, Welting TJM, Willems PC, Arts JJ, van Rhijn LW. Novel Radiopaque UHMWPE Sublaminar Wires in a Growth-Guidance System for the Treatment of Early-Onset Scoliosis: Feasibility in a Large Animal Study. Spine 39, E1503-09 (2014)

- Romme EA, Rutten EP, Geusens P, de Jong JJ, van Rietbergen B, Smeenk FW, Wouters EF, van den Bergh JP. Bone stiffness and failure load are related with clinical parameters in men with chronic obstructive pulmonary disease. $J$ Bone Miner Res 28, 2186-93 (2013)

- Geusens P, Emans PJ, de Jong JJ, van den Bergh J. NSAIDs and fracture healing. Curr Opin Rheumatol 25, 52431 (2013)

- Pereira ACdC, Castania V, de Jong JJ, Defino HL, Pitol DL, lyomasa MM, Del Bel E. Injury model by needle puncture of the Wistar rats' tail: histological and radiographic assessments. Coluna 9, 455-461 (2010)

- Heyer FH, de Jong JJ, Smeets RJ, Arts JJ, Poeze M, Geusens PP, van Rietbergen B, van den Bergh JP, Willems PC. Complex regional pain syndrome after a distal radius fracture: discrepancy between cortical and trabecular fracture healing as detected by high-resolution peripheral quantitative CT. Submitted 
- Peters M, Scharmga A, de Jong J, van Tubergen A, van den Bergh J, Loeffen D, Weijers R, van Rietbergen B, Geusens P. An Automated Algorithm for the Detection of Cortical Breaks on High-Resolution peripheral Quantitative Computed Tomography Images of Finger Joints. Submitted

- Berahmani S, Hendriks M, van den Bergh JP, de Jong JJ, Janssen D, Verdonschot N. Evaluation of interference fit and bone damage of an uncemented femoral knee implant. Submitted

- Driessen JHM, de Waard EAC, de Jong JJA, van Geel TA, Henry RMA, van Onzenoort HAW, Schram MT, Dagnelie PC, van der Kallen CJ, Sep SJS, Stehouwer CDA, Schaper NC, Koster A, Savelberg HHCM, Neef C, de Vries F, van den Bergh JPW. The association between use of metformin or insulin on volumetric bone mineral density, bone micro-architecture and bone strength of the distal radius - explorative data from The Maastricht Study. Submitted 